AUG. 31, 2020

\title{
Low-Pressure Limit of Competitive Unimolecular Reactions
}

\author{
Rui Ming Zhang, ${ }^{a, b}$ Xuefei Xu, ${ }^{*},{ }^{a}$ and Donald G. Truhlar*b, \\ ${ }^{a}$ Center for Combustion Energy, Department of Energy and Power Engineering, and Key Laboratory for Thermal \\ Science and Power Engineering of Ministry of Education, Tsinghua University, Beijing 100084, China \\ ${ }^{b}$ Department of Chemistry, Chemical Theory Center, and Minnesota Supercomputing Institute, University of \\ Minnesota, Minneapolis, Minnesota 55455-0431, USA
}

\section{TABLE OF CONTENTS}

1. Table S1-1. Eigenvalues $\left(\mathrm{s}^{-1}\right)$ for case $1 \quad \mathrm{~S}-2$

2. Table S1-2. CSE projections for case $1 \quad \mathrm{~S}-2$

3. Table S2-1. Eigenvalues $\left(\mathrm{s}^{-1}\right)$ for case $2 \quad \mathrm{~S}-3$

4. Table S2-2. CSE projections for case $2 \quad \mathrm{~S}-3$

5. Table S3-1. Eigenvalues $\left(\mathrm{s}^{-1}\right)$ for case $3 \quad \mathrm{~S}-4$

6. Table S3-2. CSE projections for case $3 \quad$ S-4

7. Table S4-1. Eigenvalues $\left(\mathrm{s}^{-1}\right)$ for case $4 \quad \mathrm{~S}-5$

8. Table S4-2. CSE projections for case $4 \quad \mathrm{~S}-5$

9. Table S5-1. Eigenvalues $\left(\mathrm{s}^{-1}\right)$ for case $5 \quad \mathrm{~S}-6$

10. Table S5-2. CSE projections for case $5 \quad$ S-6

11. Table S6-1. Eigenvalues $\left(\mathrm{s}^{-1}\right)$ for case $6 \quad \mathrm{~S}-7$

12. Table S6-2. CSE projections for case $6 \quad \mathrm{~S}-7$

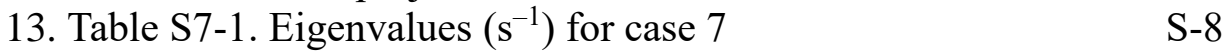

14. Table S7-2. CSE projections for case $7 \quad \mathrm{~S}-8$

15. Convergence checks and example of variation of $\langle\Delta E\rangle_{\text {down }} \quad$ S-9

16. Additional enthalpy profiles $\quad \mathrm{S}-11$

17. Note on quadruple precision $\quad \mathrm{S}-12$

18. Input file for $M E S S$ for case 1 and case $2 \quad$ S-13

19. Input file for MESS for case $3 \quad \mathrm{~S}-52$

20. Input file for $M E S S$ for case $4 \quad$ S-58

21. Input file for MESS for case $5 \quad \mathrm{~S}-64$

22. Input file for MESS for case $6 \quad \mathrm{~S}-70$

23. Input file for $M E S S$ for case $7 \quad S-76$ 
1. Table S1-1. Eigenvalues $\left(\mathrm{s}^{-1}\right)$ for case 1

\begin{tabular}{ccccccccc}
\hline$p /$ torr & $Z^{a}$ & $R^{b}$ & $E_{1}{ }^{c}$ & $E_{2}$ & $E_{3}$ & $E_{4}$ & $E_{5}$ & $E_{6}$ \\
\hline $1.00 \mathrm{E}-08$ & $7.86 \mathrm{E}-02$ & $1.29 \mathrm{E}-03$ & $3.74 \mathrm{E}-04$ & $4.65 \mathrm{E}-04$ & $4.86 \mathrm{E}-04$ & $7.91 \mathrm{E}-04$ & $1.05 \mathrm{E}-03$ & $1.26 \mathrm{E}-03$ \\
$1.00 \mathrm{E}-07$ & $7.86 \mathrm{E}-01$ & $1.23 \mathrm{E}-02$ & $3.51 \mathrm{E}-03$ & $4.44 \mathrm{E}-03$ & $4.58 \mathrm{E}-03$ & $7.53 \mathrm{E}-03$ & $9.94 \mathrm{E}-03$ & $1.21 \mathrm{E}-02$ \\
$5.00 \mathrm{E}-07$ & $3.93 \mathrm{E}+00$ & $5.86 \mathrm{E}-02$ & $1.66 \mathrm{E}-02$ & $2.12 \mathrm{E}-02$ & $2.17 \mathrm{E}-02$ & $3.58 \mathrm{E}-02$ & $4.74 \mathrm{E}-02$ & $5.84 \mathrm{E}-02$ \\
$1.00 \mathrm{E}-06$ & $7.86 \mathrm{E}+00$ & $1.15 \mathrm{E}-01$ & $3.22 \mathrm{E}-02$ & $4.15 \mathrm{E}-02$ & $4.22 \mathrm{E}-02$ & $6.99 \mathrm{E}-02$ & $9.27 \mathrm{E}-02$ & $1.14 \mathrm{E}-01$ \\
$5.00 \mathrm{E}-06$ & $3.93 \mathrm{E}+01$ & $5.43 \mathrm{E}-01$ & $1.50 \mathrm{E}-01$ & $1.95 \mathrm{E}-01$ & $1.96 \mathrm{E}-01$ & $3.25 \mathrm{E}-01$ & $4.36 \mathrm{E}-01$ & $5.36 \mathrm{E}-01$ \\
$1.00 \mathrm{E}-05$ & $7.86 \mathrm{E}+01$ & $1.06 \mathrm{E}+00$ & $2.89 \mathrm{E}-01$ & $3.76 \mathrm{E}-01$ & $3.78 \mathrm{E}-01$ & $6.27 \mathrm{E}-01$ & $8.47 \mathrm{E}-01$ & $1.04 \mathrm{E}+00$ \\
$5.00 \mathrm{E}-05$ & $3.93 \mathrm{E}+02$ & $4.92 \mathrm{E}+00$ & $1.32 \mathrm{E}+00$ & $1.70 \mathrm{E}+00$ & $1.73 \mathrm{E}+00$ & $2.84 \mathrm{E}+00$ & $3.92 \mathrm{E}+00$ & $4.77 \mathrm{E}+00$ \\
$1.00 \mathrm{E}-04$ & $7.86 \mathrm{E}+02$ & $9.50 \mathrm{E}+00$ & $2.52 \mathrm{E}+00$ & $3.24 \mathrm{E}+00$ & $3.32 \mathrm{E}+00$ & $5.41 \mathrm{E}+00$ & $7.56 \mathrm{E}+00$ & $9.15 \mathrm{E}+00$ \\
$1.00 \mathrm{E}-03$ & $7.86 \mathrm{E}+03$ & $8.25 \mathrm{E}+01$ & $2.11 \mathrm{E}+01$ & $2.67 \mathrm{E}+01$ & $2.80 \mathrm{E}+01$ & $4.45 \mathrm{E}+01$ & $6.56 \mathrm{E}+01$ & $7.80 \mathrm{E}+01$ \\
$1.00 \mathrm{E}-02$ & $7.86 \mathrm{E}+04$ & $6.92 \mathrm{E}+02$ & $1.69 \mathrm{E}+02$ & $2.09 \mathrm{E}+02$ & $2.26 \mathrm{E}+02$ & $3.46 \mathrm{E}+02$ & $5.50 \mathrm{E}+02$ & $6.41 \mathrm{E}+02$ \\
$1.00 \mathrm{E}-01$ & $7.86 \mathrm{E}+05$ & $5.65 \mathrm{E}+03$ & $1.27 \mathrm{E}+03$ & $1.55 \mathrm{E}+03$ & $1.74 \mathrm{E}+03$ & $2.50 \mathrm{E}+03$ & $4.44 \mathrm{E}+03$ & $5.07 \mathrm{E}+03$ \\
$1.00 \mathrm{E}+00$ & $7.86 \mathrm{E}+06$ & $4.52 \mathrm{E}+04$ & $8.77 \mathrm{E}+03$ & $1.06 \mathrm{E}+04$ & $1.28 \mathrm{E}+04$ & $1.65 \mathrm{E}+04$ & $3.40 \mathrm{E}+04$ & $3.84 \mathrm{E}+04$ \\
$1.00 \mathrm{E}+01$ & $7.86 \mathrm{E}+07$ & $3.44 \mathrm{E}+05$ & $5.35 \mathrm{E}+04$ & $6.50 \mathrm{E}+04$ & $9.03 \mathrm{E}+04$ & $9.66 \mathrm{E}+04$ & $2.45 \mathrm{E}+05$ & $2.76 \mathrm{E}+05$ \\
$1.00 \mathrm{E}+02$ & $7.86 \mathrm{E}+08$ & $2.24 \mathrm{E}+06$ & $2.65 \mathrm{E}+05$ & $3.42 \mathrm{E}+05$ & $4.95 \mathrm{E}+05$ & $5.93 \mathrm{E}+05$ & $1.63 \mathrm{E}+06$ & $1.88 \mathrm{E}+06$ \\
$1.00 \mathrm{E}+03$ & $7.86 \mathrm{E}+09$ & $1.26 \mathrm{E}+07$ & $9.48 \mathrm{E}+05$ & $1.41 \mathrm{E}+06$ & $2.17 \mathrm{E}+06$ & $3.48 \mathrm{E}+06$ & $1.03 \mathrm{E}+07$ & $1.22 \mathrm{E}+07$ \\
\hline
\end{tabular}

${ }^{a}$ Collision frequency in units of $\mathrm{s}^{-1}$

${ }^{b}$ Minimum IERE eigenvalue

${ }^{c}$ CSE eigenvalues. The number of CSEs equals the number of wells.

2. Table S1-2. CSE projections for case 1

\begin{tabular}{ccccccc}
\hline$p /$ torr & $P_{1}{ }^{a}$ & $P_{2}$ & $P_{3}$ & $P_{4}$ & $P_{5}$ & $P_{6}$ \\
\hline $1.00 \mathrm{E}-08$ & $9.74 \mathrm{E}-01$ & $9.84 \mathrm{E}-01$ & $9.87 \mathrm{E}-01$ & $9.97 \mathrm{E}-01$ & $9.92 \mathrm{E}-01$ & $9.95 \mathrm{E}-01$ \\
$1.00 \mathrm{E}-07$ & $9.70 \mathrm{E}-01$ & $9.82 \mathrm{E}-01$ & $9.85 \mathrm{E}-01$ & $9.96 \mathrm{E}-01$ & $9.91 \mathrm{E}-01$ & $9.94 \mathrm{E}-01$ \\
$5.00 \mathrm{E}-07$ & $9.65 \mathrm{E}-01$ & $9.79 \mathrm{E}-01$ & $9.82 \mathrm{E}-01$ & $9.96 \mathrm{E}-01$ & $9.90 \mathrm{E}-01$ & $9.94 \mathrm{E}-01$ \\
$1.00 \mathrm{E}-06$ & $9.63 \mathrm{E}-01$ & $9.78 \mathrm{E}-01$ & $9.81 \mathrm{E}-01$ & $9.95 \mathrm{E}-01$ & $9.89 \mathrm{E}-01$ & $9.94 \mathrm{E}-01$ \\
$5.00 \mathrm{E}-06$ & $9.56 \mathrm{E}-01$ & $9.74 \mathrm{E}-01$ & $9.76 \mathrm{E}-01$ & $9.94 \mathrm{E}-01$ & $9.88 \mathrm{E}-01$ & $9.92 \mathrm{E}-01$ \\
$1.00 \mathrm{E}-05$ & $9.53 \mathrm{E}-01$ & $9.74 \mathrm{E}-01$ & $9.73 \mathrm{E}-01$ & $9.93 \mathrm{E}-01$ & $9.87 \mathrm{E}-01$ & $9.92 \mathrm{E}-01$ \\
$5.00 \mathrm{E}-05$ & $9.42 \mathrm{E}-01$ & $9.67 \mathrm{E}-01$ & $9.66 \mathrm{E}-01$ & $9.91 \mathrm{E}-01$ & $9.84 \mathrm{E}-01$ & $9.89 \mathrm{E}-01$ \\
$1.00 \mathrm{E}-04$ & $9.36 \mathrm{E}-01$ & $9.62 \mathrm{E}-01$ & $9.62 \mathrm{E}-01$ & $9.89 \mathrm{E}-01$ & $9.82 \mathrm{E}-01$ & $9.88 \mathrm{E}-01$ \\
$1.00 \mathrm{E}-03$ & $9.09 \mathrm{E}-01$ & $9.42 \mathrm{E}-01$ & $9.44 \mathrm{E}-01$ & $9.82 \mathrm{E}-01$ & $9.75 \mathrm{E}-01$ & $9.81 \mathrm{E}-01$ \\
$1.00 \mathrm{E}-02$ & $8.65 \mathrm{E}-01$ & $9.05 \mathrm{E}-01$ & $9.13 \mathrm{E}-01$ & $9.64 \mathrm{E}-01$ & $9.64 \mathrm{E}-01$ & $9.71 \mathrm{E}-01$ \\
$1.00 \mathrm{E}-01$ & $7.92 \mathrm{E}-01$ & $8.40 \mathrm{E}-01$ & $8.63 \mathrm{E}-01$ & $9.25 \mathrm{E}-01$ & $9.48 \mathrm{E}-01$ & $9.54 \mathrm{E}-01$ \\
$1.00 \mathrm{E}+00$ & $6.72 \mathrm{E}-01$ & $7.29 \mathrm{E}-01$ & $7.87 \mathrm{E}-01$ & $8.38 \mathrm{E}-01$ & $9.27 \mathrm{E}-01$ & $9.31 \mathrm{E}-01$ \\
$1.00 \mathrm{E}+01$ & $4.90 \mathrm{E}-01$ & $5.57 \mathrm{E}-01$ & $6.78 \mathrm{E}-01$ & $6.72 \mathrm{E}-01$ & $9.07 \mathrm{E}-01$ & $9.10 \mathrm{E}-01$ \\
$1.00 \mathrm{E}+02$ & $2.62 \mathrm{E}-01$ & $3.36 \mathrm{E}-01$ & $4.38 \mathrm{E}-01$ & $5.32 \mathrm{E}-01$ & $9.14 \mathrm{E}-01$ & $9.10 \mathrm{E}-01$ \\
$1.00 \mathrm{E}+03$ & $7.76 \mathrm{E}-02$ & $1.32 \mathrm{E}-01$ & $2.10 \mathrm{E}-01$ & $3.54 \mathrm{E}-01$ & $9.51 \mathrm{E}-01$ & $9.41 \mathrm{E}-01$ \\
\hline
\end{tabular}

${ }^{a} \mathrm{CSE}$ eigenvector projection on the relaxation subspace 
3. Table S2-1. Eigenvalues $\left(\mathrm{s}^{-1}\right)$ for case 2

\begin{tabular}{ccccccccc}
\hline$p /$ torr & $Z^{a}$ & $R^{b}$ & $E_{1}{ }^{c}$ & $E_{2}$ & $E_{3}$ & $E_{4}$ & $E_{5}$ & $E_{6}$ \\
\hline $1.00 \mathrm{E}-08$ & $1.61 \mathrm{E}-01$ & $1.22 \mathrm{E}-03$ & $1.48 \mathrm{E}-07$ & $7.21 \mathrm{E}-07$ & $8.24 \mathrm{E}-07$ & $1.22 \mathrm{E}-05$ & $1.59 \mathrm{E}-04$ & $2.65 \mathrm{E}-04$ \\
$1.00 \mathrm{E}-07$ & $1.61 \mathrm{E}+00$ & $1.21 \mathrm{E}-02$ & $9.36 \mathrm{E}-07$ & $5.34 \mathrm{E}-06$ & $5.63 \mathrm{E}-06$ & $9.52 \mathrm{E}-05$ & $1.54 \mathrm{E}-03$ & $2.57 \mathrm{E}-03$ \\
$5.00 \mathrm{E}-07$ & $8.06 \mathrm{E}+00$ & $6.01 \mathrm{E}-02$ & $3.12 \mathrm{E}-06$ & $1.96 \mathrm{E}-05$ & $2.00 \mathrm{E}-05$ & $3.69 \mathrm{E}-04$ & $7.34 \mathrm{E}-03$ & $1.23 \mathrm{E}-02$ \\
$1.00 \mathrm{E}-06$ & $1.61 \mathrm{E}+01$ & $1.20 \mathrm{E}-01$ & $5.12 \mathrm{E}-06$ & $3.26 \mathrm{E}-05$ & $3.44 \mathrm{E}-05$ & $6.45 \mathrm{E}-04$ & $1.42 \mathrm{E}-02$ & $2.38 \mathrm{E}-02$ \\
$5.00 \mathrm{E}-06$ & $8.06 \mathrm{E}+01$ & $5.96 \mathrm{E}-01$ & $1.51 \mathrm{E}-05$ & $9.85 \mathrm{E}-05$ & $1.13 \mathrm{E}-04$ & $2.21 \mathrm{E}-03$ & $6.46 \mathrm{E}-02$ & $1.08 \mathrm{E}-01$ \\
$1.00 \mathrm{E}-05$ & $1.61 \mathrm{E}+02$ & $1.19 \mathrm{E}+00$ & $2.32 \mathrm{E}-05$ & $1.53 \mathrm{E}-04$ & $1.83 \mathrm{E}-04$ & $3.62 \mathrm{E}-03$ & $1.22 \mathrm{E}-01$ & $2.05 \mathrm{E}-01$ \\
$5.00 \mathrm{E}-05$ & $8.06 \mathrm{E}+02$ & $5.93 \mathrm{E}+00$ & $5.82 \mathrm{E}-05$ & $3.86 \mathrm{E}-04$ & $5.14 \mathrm{E}-04$ & $1.05 \mathrm{E}-02$ & $5.19 \mathrm{E}-01$ & $8.75 \mathrm{E}-01$ \\
$1.00 \mathrm{E}-04$ & $1.61 \mathrm{E}+03$ & $1.18 \mathrm{E}+01$ & $8.31 \mathrm{E}-05$ & $5.51 \mathrm{E}-04$ & $7.69 \mathrm{E}-04$ & $1.59 \mathrm{E}-02$ & $9.52 \mathrm{E}-01$ & $1.61 \mathrm{E}+00$ \\
$1.00 \mathrm{E}-03$ & $1.61 \mathrm{E}+04$ & $1.18 \mathrm{E}+02$ & $2.24 \mathrm{E}-04$ & $1.46 \mathrm{E}-03$ & $2.39 \mathrm{E}-03$ & $5.13 \mathrm{E}-02$ & $6.50 \mathrm{E}+00$ & $1.11 \mathrm{E}+01$ \\
$1.00 \mathrm{E}-02$ & $1.61 \mathrm{E}+05$ & $1.18 \mathrm{E}+03$ & $4.38 \mathrm{E}-04$ & $2.79 \mathrm{E}-03$ & $5.41 \mathrm{E}-03$ & $1.15 \mathrm{E}-01$ & $3.68 \mathrm{E}+01$ & $6.29 \mathrm{E}+01$ \\
$1.00 \mathrm{E}-01$ & $1.61 \mathrm{E}+06$ & $1.17 \mathrm{E}+04$ & $6.31 \mathrm{E}-04$ & $3.93 \mathrm{E}-03$ & $9.12 \mathrm{E}-03$ & $1.77 \mathrm{E}-01$ & $1.57 \mathrm{E}+02$ & $2.70 \mathrm{E}+02$ \\
$1.00 \mathrm{E}+00$ & $1.61 \mathrm{E}+07$ & $1.17 \mathrm{E}+05$ & $7.32 \mathrm{E}-04$ & $4.45 \mathrm{E}-03$ & $1.22 \mathrm{E}-02$ & $2.06 \mathrm{E}-01$ & $4.55 \mathrm{E}+02$ & $7.71 \mathrm{E}+02$ \\
$1.00 \mathrm{E}+01$ & $1.61 \mathrm{E}+08$ & $1.17 \mathrm{E}+06$ & $7.67 \mathrm{E}-04$ & $4.57 \mathrm{E}-03$ & $1.39 \mathrm{E}-02$ & $2.14 \mathrm{E}-01$ & $8.23 \mathrm{E}+02$ & $1.36 \mathrm{E}+03$ \\
$1.00 \mathrm{E}+02$ & $1.61 \mathrm{E}+09$ & $1.17 \mathrm{E}+07$ & $7.75 \mathrm{E}-04$ & $4.59 \mathrm{E}-03$ & $1.44 \mathrm{E}-02$ & $2.15 \mathrm{E}-01$ & $1.01 \mathrm{E}+03$ & $1.62 \mathrm{E}+03$ \\
$1.00 \mathrm{E}+03$ & $1.61 \mathrm{E}+10$ & $1.17 \mathrm{E}+08$ & $7.76 \mathrm{E}-04$ & $4.60 \mathrm{E}-03$ & $1.45 \mathrm{E}-02$ & $2.15 \mathrm{E}-01$ & $1.05 \mathrm{E}+03$ & $1.67 \mathrm{E}+03$ \\
\hline
\end{tabular}

${ }^{a}$ Collision frequency in units of $\mathrm{s}^{-1}$

${ }^{b}$ Minimum IERE eigenvalue

${ }^{c}$ CSE eigenvalues. The number of CSEs equals the number of wells.

4. Table S2-2. CSE projections for case 2

\begin{tabular}{ccccccc}
\hline$p /$ torr & $P_{1}{ }^{a}$ & $P_{2}$ & $P_{3}$ & $P_{4}$ & $P_{5}$ & $P_{6}$ \\
\hline $1.00 \mathrm{E}-08$ & $4.68 \mathrm{E}-05$ & $2.58 \mathrm{E}-04$ & $3.01 \mathrm{E}-04$ & $5.87 \mathrm{E}-03$ & $9.10 \mathrm{E}-02$ & $1.47 \mathrm{E}-01$ \\
$1.00 \mathrm{E}-07$ & $2.79 \mathrm{E}-05$ & $1.83 \mathrm{E}-04$ & $1.93 \mathrm{E}-04$ & $4.40 \mathrm{E}-03$ & $8.84 \mathrm{E}-02$ & $1.43 \mathrm{E}-01$ \\
$5.00 \mathrm{E}-07$ & $1.76 \mathrm{E}-05$ & $1.26 \mathrm{E}-04$ & $1.30 \mathrm{E}-04$ & $3.25 \mathrm{E}-03$ & $8.40 \mathrm{E}-02$ & $1.36 \mathrm{E}-01$ \\
$1.00 \mathrm{E}-06$ & $1.40 \mathrm{E}-05$ & $1.02 \mathrm{E}-04$ & $1.09 \mathrm{E}-04$ & $2.77 \mathrm{E}-03$ & $8.14 \mathrm{E}-02$ & $1.32 \mathrm{E}-01$ \\
$5.00 \mathrm{E}-06$ & $7.53 \mathrm{E}-06$ & $5.57 \mathrm{E}-05$ & $6.68 \mathrm{E}-05$ & $1.75 \mathrm{E}-03$ & $7.36 \mathrm{E}-02$ & $1.21 \mathrm{E}-01$ \\
$1.00 \mathrm{E}-05$ & $5.55 \mathrm{E}-06$ & $4.11 \mathrm{E}-05$ & $5.20 \mathrm{E}-05$ & $1.37 \mathrm{E}-03$ & $6.94 \mathrm{E}-02$ & $1.14 \mathrm{E}-01$ \\
$5.00 \mathrm{E}-05$ & $2.44 \mathrm{E}-06$ & $1.79 \mathrm{E}-05$ & $2.59 \mathrm{E}-05$ & $6.99 \mathrm{E}-04$ & $5.84 \mathrm{E}-02$ & $9.75 \mathrm{E}-02$ \\
$1.00 \mathrm{E}-04$ & $1.63 \mathrm{E}-06$ & $1.18 \mathrm{E}-05$ & $1.82 \mathrm{E}-05$ & $4.94 \mathrm{E}-04$ & $5.31 \mathrm{E}-02$ & $8.92 \mathrm{E}-02$ \\
$1.00 \mathrm{E}-03$ & $3.23 \mathrm{E}-07$ & $2.23 \mathrm{E}-06$ & $4.28 \mathrm{E}-06$ & $1.16 \mathrm{E}-04$ & $3.46 \mathrm{E}-02$ & $5.96 \mathrm{E}-02$ \\
$1.00 \mathrm{E}-02$ & $3.89 \mathrm{E}-08$ & $2.52 \mathrm{E}-07$ & $6.50 \mathrm{E}-07$ & $1.55 \mathrm{E}-05$ & $1.76 \mathrm{E}-02$ & $3.10 \mathrm{E}-02$ \\
$1.00 \mathrm{E}-01$ & $2.58 \mathrm{E}-09$ & $1.54 \mathrm{E}-08$ & $6.46 \mathrm{E}-08$ & $1.01 \mathrm{E}-06$ & $6.00 \mathrm{E}-03$ & $1.07 \mathrm{E}-02$ \\
$1.00 \mathrm{E}+00$ & $1.03 \mathrm{E}-10$ & $4.76 \mathrm{E}-10$ & $4.20 \mathrm{E}-09$ & $3.10 \mathrm{E}-08$ & $1.10 \mathrm{E}-03$ & $1.92 \mathrm{E}-03$ \\
$1.00 \mathrm{E}+01$ & $2.76 \mathrm{E}-12$ & $8.35 \mathrm{E}-12$ & $1.65 \mathrm{E}-10$ & $6.19 \mathrm{E}-10$ & $8.34 \mathrm{E}-05$ & $1.33 \mathrm{E}-04$ \\
$1.00 \mathrm{E}+02$ & $4.55 \mathrm{E}-14$ & $1.46 \mathrm{E}-13$ & $3.75 \mathrm{E}-12$ & $1.21 \mathrm{E}-11$ & $2.28 \mathrm{E}-06$ & $3.20 \mathrm{E}-06$ \\
$1.00 \mathrm{E}+03$ & $1.33 \mathrm{E}-15$ & $2.33 \mathrm{E}-15$ & $5.32 \mathrm{E}-14$ & $1.63 \mathrm{E}-13$ & $3.02 \mathrm{E}-08$ & $3.97 \mathrm{E}-08$ \\
\hline
\end{tabular}

${ }^{a} \mathrm{CSE}$ eigenvector projection on the relaxation subspace 
5. Table S3-1. Eigenvalues $\left(\mathrm{s}^{-1}\right)$ for case 3

\begin{tabular}{cccc}
\hline$p /$ torr & $Z^{a}$ & $R^{b}$ & $E_{1}{ }^{c}$ \\
\hline $1.00 \mathrm{E}-07$ & $1.02 \mathrm{E}+00$ & $7.50 \mathrm{E}-03$ & $1.20 \mathrm{E}-03$ \\
$5.00 \mathrm{E}-07$ & $5.11 \mathrm{E}+00$ & $3.57 \mathrm{E}-02$ & $5.50 \mathrm{E}-03$ \\
$1.00 \mathrm{E}-06$ & $1.02 \mathrm{E}+01$ & $6.98 \mathrm{E}-02$ & $1.05 \mathrm{E}-02$ \\
$5.00 \mathrm{E}-06$ & $5.11 \mathrm{E}+01$ & $3.28 \mathrm{E}-01$ & $4.67 \mathrm{E}-02$ \\
$1.00 \mathrm{E}-05$ & $1.02 \mathrm{E}+02$ & $6.36 \mathrm{E}-01$ & $8.82 \mathrm{E}-02$ \\
$5.00 \mathrm{E}-05$ & $5.11 \mathrm{E}+02$ & $2.94 \mathrm{E}+00$ & $3.77 \mathrm{E}-01$ \\
$1.00 \mathrm{E}-04$ & $1.02 \mathrm{E}+03$ & $5.66 \mathrm{E}+00$ & $6.97 \mathrm{E}-01$ \\
$1.00 \mathrm{E}-03$ & $1.02 \mathrm{E}+04$ & $4.91 \mathrm{E}+01$ & $5.11 \mathrm{E}+00$ \\
$1.00 \mathrm{E}-02$ & $1.02 \mathrm{E}+05$ & $4.14 \mathrm{E}+02$ & $3.38 \mathrm{E}+01$ \\
$1.00 \mathrm{E}-01$ & $1.02 \mathrm{E}+06$ & $3.41 \mathrm{E}+03$ & $1.95 \mathrm{E}+02$ \\
$1.00 \mathrm{E}+00$ & $1.02 \mathrm{E}+07$ & $2.77 \mathrm{E}+04$ & $9.27 \mathrm{E}+02$ \\
$1.00 \mathrm{E}+01$ & $1.02 \mathrm{E}+08$ & $2.28 \mathrm{E}+05$ & $3.34 \mathrm{E}+03$ \\
$1.00 \mathrm{E}+02$ & $1.02 \mathrm{E}+09$ & $1.95 \mathrm{E}+06$ & $8.29 \mathrm{E}+03$ \\
$1.00 \mathrm{E}+04$ & $1.02 \mathrm{E}+11$ & $1.73 \mathrm{E}+08$ & $1.57 \mathrm{E}+04$ \\
\hline
\end{tabular}

\section{Table S3-2. CSE projections for case 3}

\begin{tabular}{cc}
\multicolumn{1}{c}{$p /$ torr } & $P_{1}{ }^{a}$ \\
\cline { 2 - 3 } $1.00 \mathrm{E}-07$ & $4.02 \mathrm{E}-01$ \\
$5.00 \mathrm{E}-07$ & $3.76 \mathrm{E}-01$ \\
$1.00 \mathrm{E}-06$ & $3.64 \mathrm{E}-01$ \\
$5.00 \mathrm{E}-06$ & $3.31 \mathrm{E}-01$ \\
$1.00 \mathrm{E}-05$ & $3.16 \mathrm{E}-01$ \\
$5.00 \mathrm{E}-05$ & $2.76 \mathrm{E}-01$ \\
$1.00 \mathrm{E}-04$ & $2.58 \mathrm{E}-01$ \\
$1.00 \mathrm{E}-03$ & $1.93 \mathrm{E}-01$ \\
$1.00 \mathrm{E}-02$ & $1.27 \mathrm{E}-01$ \\
$1.00 \mathrm{E}-01$ & $6.91 \mathrm{E}-02$ \\
$1.00 \mathrm{E}+00$ & $2.83 \mathrm{E}-02$ \\
$1.00 \mathrm{E}+01$ & $7.55 \mathrm{E}-03$ \\
$1.00 \mathrm{E}+02$ & $1.07 \mathrm{E}-03$ \\
$1.00 \mathrm{E}+04$ & $1.27 \mathrm{E}-06$ \\
\hline
\end{tabular}

${ }^{a} \mathrm{CSE}$ eigenvector projection on the relaxation subspace 


\section{Table S4-1. Eigenvalues $\left(s^{-1}\right)$ for case 4}

\begin{tabular}{cccccc}
\hline$p /$ torr & $Z^{a}$ & $R^{b}$ & $E_{1}{ }^{c}$ & $E_{2}$ & $E_{3}$ \\
\hline $1.00 \mathrm{E}-08$ & $1.61 \mathrm{E}-01$ & $1.18 \mathrm{E}-03$ & $7.99 \mathrm{E}-12$ & $1.02 \mathrm{E}-07$ & $7.73 \mathrm{E}-05$ \\
$7.00 \mathrm{E}-08$ & $1.13 \mathrm{E}+00$ & $8.27 \mathrm{E}-03$ & $2.11 \mathrm{E}-11$ & $4.95 \mathrm{E}-07$ & $5.22 \mathrm{E}-04$ \\
$1.00 \mathrm{E}-07$ & $1.61 \mathrm{E}+00$ & $1.18 \mathrm{E}-02$ & $2.47 \mathrm{E}-11$ & $6.64 \mathrm{E}-07$ & $7.36 \mathrm{E}-04$ \\
$7.00 \mathrm{E}-07$ & $1.13 \mathrm{E}+01$ & $8.27 \mathrm{E}-02$ & $5.14 \mathrm{E}-11$ & $2.81 \mathrm{E}-06$ & $4.65 \mathrm{E}-03$ \\
$1.00 \mathrm{E}-06$ & $1.61 \mathrm{E}+01$ & $1.18 \mathrm{E}-01$ & $5.74 \mathrm{E}-11$ & $3.61 \mathrm{E}-06$ & $6.48 \mathrm{E}-03$ \\
$5.00 \mathrm{E}-06$ & $8.06 \mathrm{E}+01$ & $5.90 \mathrm{E}-01$ & $8.72 \mathrm{E}-11$ & $1.05 \mathrm{E}-05$ & $2.79 \mathrm{E}-02$ \\
$1.00 \mathrm{E}-05$ & $1.61 \mathrm{E}+02$ & $1.18 \mathrm{E}+00$ & $9.99 \mathrm{E}-11$ & $1.62 \mathrm{E}-05$ & $5.13 \mathrm{E}-02$ \\
$5.00 \mathrm{E}-05$ & $8.06 \mathrm{E}+02$ & $5.87 \mathrm{E}+00$ & $1.26 \mathrm{E}-10$ & $4.00 \mathrm{E}-05$ & $2.01 \mathrm{E}-01$ \\
$1.00 \mathrm{E}-04$ & $1.61 \mathrm{E}+03$ & $1.17 \mathrm{E}+01$ & $1.34 \mathrm{E}-10$ & $5.67 \mathrm{E}-05$ & $3.54 \mathrm{E}-01$ \\
$1.00 \mathrm{E}-03$ & $1.61 \mathrm{E}+04$ & $1.17 \mathrm{E}+02$ & $1.50 \mathrm{E}-10$ & $1.48 \mathrm{E}-04$ & $2.01 \mathrm{E}+00$ \\
$1.00 \mathrm{E}-02$ & $1.61 \mathrm{E}+05$ & $1.17 \mathrm{E}+03$ & $1.53 \mathrm{E}-10$ & $2.79 \mathrm{E}-04$ & $8.66 \mathrm{E}+00$ \\
$1.00 \mathrm{E}+00$ & $1.61 \mathrm{E}+07$ & $1.17 \mathrm{E}+05$ & $1.54 \mathrm{E}-10$ & $4.38 \mathrm{E}-04$ & $4.77 \mathrm{E}+01$ \\
\hline
\end{tabular}

${ }^{a}$ Collision frequency in units of $\mathrm{s}^{-1}$

${ }^{b}$ Minimum IERE eigenvalue

${ }^{c}$ CSE eigenvalues. The number of CSEs equals the number of wells.

\section{Table S4-2. CSE projections for case 4}

\begin{tabular}{cccc}
\hline$p /$ torr & $P_{1}{ }^{a}$ & $P_{2}$ & $P_{3}$ \\
\hline $1.00 \mathrm{E}-08$ & $1.34 \mathrm{E}-09$ & $3.19 \mathrm{E}-05$ & $4.42 \mathrm{E}-02$ \\
$7.00 \mathrm{E}-08$ & $4.25 \mathrm{E}-10$ & $2.10 \mathrm{E}-05$ & $4.24 \mathrm{E}-02$ \\
$1.00 \mathrm{E}-07$ & $3.35 \mathrm{E}-10$ & $1.92 \mathrm{E}-05$ & $4.19 \mathrm{E}-02$ \\
$7.00 \mathrm{E}-07$ & $7.67 \mathrm{E}-11$ & $1.08 \mathrm{E}-05$ & $3.74 \mathrm{E}-02$ \\
$1.00 \mathrm{E}-06$ & $5.66 \mathrm{E}-11$ & $9.57 \mathrm{E}-06$ & $3.64 \mathrm{E}-02$ \\
$5.00 \mathrm{E}-06$ & $1.24 \mathrm{E}-11$ & $5.12 \mathrm{E}-06$ & $3.07 \mathrm{E}-02$ \\
$1.00 \mathrm{E}-05$ & $5.97 \mathrm{E}-12$ & $3.75 \mathrm{E}-06$ & $2.80 \mathrm{E}-02$ \\
$5.00 \mathrm{E}-05$ & $9.00 \mathrm{E}-13$ & $1.62 \mathrm{E}-06$ & $2.11 \mathrm{E}-02$ \\
$1.00 \mathrm{E}-04$ & $3.64 \mathrm{E}-13$ & $1.07 \mathrm{E}-06$ & $1.82 \mathrm{E}-02$ \\
$1.00 \mathrm{E}-03$ & $1.24 \mathrm{E}-14$ & $2.02 \mathrm{E}-07$ & $9.17 \mathrm{E}-03$ \\
$1.00 \mathrm{E}-02$ & $3.33 \mathrm{E}-16$ & $2.28 \mathrm{E}-08$ & $3.14 \mathrm{E}-03$ \\
$1.00 \mathrm{E}+00$ & $8.88 \mathrm{E}-16$ & $4.14 \mathrm{E}-11$ & $5.03 \mathrm{E}-05$ \\
\hline
\end{tabular}

${ }^{a} \mathrm{CSE}$ eigenvector projection on the relaxation subspace 
9. Table S5-1. Eigenvalues $\left(s^{-1}\right)$ for case 5

\begin{tabular}{cccccc}
\hline$p /$ torr & $Z^{a}$ & $R^{b}$ & $E_{1}{ }^{c}$ & $E_{2}$ & $E_{3}$ \\
\hline $1.00 \mathrm{E}-08$ & $1.61 \mathrm{E}-01$ & $1.19 \mathrm{E}-03$ & $2.89 \mathrm{E}-09$ & $5.34 \mathrm{E}-05$ & $7.83 \mathrm{E}-05$ \\
$7.00 \mathrm{E}-08$ & $1.13 \mathrm{E}+00$ & $8.29 \mathrm{E}-03$ & $1.16 \mathrm{E}-08$ & $3.33 \mathrm{E}-04$ & $5.27 \mathrm{E}-04$ \\
$1.00 \mathrm{E}-07$ & $1.61 \mathrm{E}+00$ & $1.18 \mathrm{E}-02$ & $1.48 \mathrm{E}-08$ & $4.62 \mathrm{E}-04$ & $7.44 \mathrm{E}-04$ \\
$7.00 \mathrm{E}-07$ & $1.13 \mathrm{E}+01$ & $8.28 \mathrm{E}-02$ & $5.06 \mathrm{E}-08$ & $2.65 \mathrm{E}-03$ & $4.69 \mathrm{E}-03$ \\
$1.00 \mathrm{E}-06$ & $1.61 \mathrm{E}+01$ & $1.18 \mathrm{E}-01$ & $6.23 \mathrm{E}-08$ & $3.61 \mathrm{E}-03$ & $6.52 \mathrm{E}-03$ \\
$5.00 \mathrm{E}-06$ & $8.06 \mathrm{E}+01$ & $5.91 \mathrm{E}-01$ & $1.47 \mathrm{E}-07$ & $1.41 \mathrm{E}-02$ & $2.80 \mathrm{E}-02$ \\
$1.00 \mathrm{E}-05$ & $1.61 \mathrm{E}+02$ & $1.18 \mathrm{E}+00$ & $2.05 \mathrm{E}-07$ & $2.47 \mathrm{E}-02$ & $5.16 \mathrm{E}-02$ \\
$5.00 \mathrm{E}-05$ & $8.06 \mathrm{E}+02$ & $5.91 \mathrm{E}+00$ & $3.99 \mathrm{E}-07$ & $8.53 \mathrm{E}-02$ & $2.02 \mathrm{E}-01$ \\
$1.00 \mathrm{E}-04$ & $1.61 \mathrm{E}+03$ & $1.18 \mathrm{E}+01$ & $5.07 \mathrm{E}-07$ & $1.41 \mathrm{E}-01$ & $3.55 \mathrm{E}-01$ \\
$1.00 \mathrm{E}-03$ & $1.61 \mathrm{E}+04$ & $1.18 \mathrm{E}+02$ & $9.26 \mathrm{E}-07$ & $6.14 \mathrm{E}-01$ & $2.02 \mathrm{E}+00$ \\
$1.00 \mathrm{E}-02$ & $1.61 \mathrm{E}+05$ & $1.18 \mathrm{E}+03$ & $1.27 \mathrm{E}-06$ & $1.87 \mathrm{E}+00$ & $8.79 \mathrm{E}+00$ \\
$1.00 \mathrm{E}-01$ & $1.61 \mathrm{E}+06$ & $1.18 \mathrm{E}+04$ & $1.43 \mathrm{E}-06$ & $3.66 \mathrm{E}+00$ & $2.63 \mathrm{E}+01$ \\
$1.00 \mathrm{E}+00$ & $1.61 \mathrm{E}+07$ & $1.18 \mathrm{E}+05$ & $1.47 \mathrm{E}-06$ & $4.84 \mathrm{E}+00$ & $5.04 \mathrm{E}+01$ \\
\hline
\end{tabular}

${ }^{a}$ Collision frequency in units of $\mathrm{s}^{-1}$

${ }^{b}$ Minimum IERE eigenvalue

${ }^{c} \mathrm{CSE}$ eigenvalues. The number of CSEs equals the number of wells.

\section{Table S5-2. CSE projections for case 5}

\begin{tabular}{cccc}
\hline$p$ /torr & $P_{1}{ }^{a}$ & $P_{2}$ & $P_{3}$ \\
\hline $1.00 \mathrm{E}-08$ & $7.06 \mathrm{E}-07$ & $2.98 \mathrm{E}-02$ & $4.48 \mathrm{E}-02$ \\
$7.00 \mathrm{E}-08$ & $3.80 \mathrm{E}-07$ & $2.61 \mathrm{E}-02$ & $4.29 \mathrm{E}-02$ \\
$1.00 \mathrm{E}-07$ & $3.33 \mathrm{E}-07$ & $2.53 \mathrm{E}-02$ & $4.23 \mathrm{E}-02$ \\
$7.00 \mathrm{E}-07$ & $1.46 \mathrm{E}-07$ & $2.01 \mathrm{E}-02$ & $3.77 \mathrm{E}-02$ \\
$1.00 \mathrm{E}-06$ & $1.23 \mathrm{E}-07$ & $1.91 \mathrm{E}-02$ & $3.66 \mathrm{E}-02$ \\
$5.00 \mathrm{E}-06$ & $5.08 \mathrm{E}-08$ & $1.43 \mathrm{E}-02$ & $3.09 \mathrm{E}-02$ \\
$1.00 \mathrm{E}-05$ & $3.29 \mathrm{E}-08$ & $1.23 \mathrm{E}-02$ & $2.81 \mathrm{E}-02$ \\
$5.00 \mathrm{E}-05$ & $1.04 \mathrm{E}-08$ & $7.84 \mathrm{E}-03$ & $2.12 \mathrm{E}-02$ \\
$1.00 \mathrm{E}-04$ & $5.95 \mathrm{E}-09$ & $6.18 \mathrm{E}-03$ & $1.82 \mathrm{E}-02$ \\
$1.00 \mathrm{E}-03$ & $6.60 \mathrm{E}-10$ & $2.16 \mathrm{E}-03$ & $9.25 \mathrm{E}-03$ \\
$1.00 \mathrm{E}-02$ & $4.06 \mathrm{E}-11$ & $4.39 \mathrm{E}-04$ & $3.21 \mathrm{E}-03$ \\
$1.00 \mathrm{E}-01$ & $1.30 \mathrm{E}-12$ & $4.18 \mathrm{E}-05$ & $6.32 \mathrm{E}-04$ \\
$1.00 \mathrm{E}+00$ & $3.32 \mathrm{E}-14$ & $1.59 \mathrm{E}-06$ & $5.62 \mathrm{E}-05$ \\
\hline
\end{tabular}

${ }^{a} \mathrm{CSE}$ eigenvector projection on the relaxation subspace 
11. Table S6-1. Eigenvalues $\left(\mathrm{s}^{-1}\right)$ for case 6

\begin{tabular}{cccccc}
\hline$p$ torr & $Z^{a}$ & $R^{b}$ & $E_{1}{ }^{c}$ & $E_{2}$ & $E_{3}$ \\
\hline $1.00 \mathrm{E}-08$ & $1.61 \mathrm{E}-01$ & $1.19 \mathrm{E}-03$ & $2.83 \mathrm{E}-09$ & $8.16 \mathrm{E}-05$ & $2.58 \mathrm{E}-04$ \\
$7.00 \mathrm{E}-08$ & $1.13 \mathrm{E}+00$ & $8.29 \mathrm{E}-03$ & $1.15 \mathrm{E}-08$ & $5.41 \mathrm{E}-04$ & $1.79 \mathrm{E}-03$ \\
$1.00 \mathrm{E}-07$ & $1.61 \mathrm{E}+00$ & $1.18 \mathrm{E}-02$ & $1.48 \mathrm{E}-08$ & $7.61 \mathrm{E}-04$ & $2.54 \mathrm{E}-03$ \\
$7.00 \mathrm{E}-07$ & $1.13 \mathrm{E}+01$ & $8.28 \mathrm{E}-02$ & $5.06 \mathrm{E}-08$ & $4.70 \mathrm{E}-03$ & $1.69 \mathrm{E}-02$ \\
$1.00 \mathrm{E}-06$ & $1.61 \mathrm{E}+01$ & $1.18 \mathrm{E}-01$ & $6.23 \mathrm{E}-08$ & $6.51 \mathrm{E}-03$ & $2.38 \mathrm{E}-02$ \\
$5.00 \mathrm{E}-06$ & $8.06 \mathrm{E}+01$ & $5.91 \mathrm{E}-01$ & $1.47 \mathrm{E}-07$ & $2.73 \mathrm{E}-02$ & $1.08 \mathrm{E}-01$ \\
$1.00 \mathrm{E}-05$ & $1.61 \mathrm{E}+02$ & $1.18 \mathrm{E}+00$ & $2.05 \mathrm{E}-07$ & $4.97 \mathrm{E}-02$ & $2.05 \mathrm{E}-01$ \\
$5.00 \mathrm{E}-05$ & $8.06 \mathrm{E}+02$ & $5.91 \mathrm{E}+00$ & $3.99 \mathrm{E}-07$ & $1.88 \mathrm{E}-01$ & $8.75 \mathrm{E}-01$ \\
$1.00 \mathrm{E}-04$ & $1.61 \mathrm{E}+03$ & $1.18 \mathrm{E}+01$ & $5.08 \mathrm{E}-07$ & $3.25 \mathrm{E}-01$ & $1.61 \mathrm{E}+00$ \\
$1.00 \mathrm{E}-03$ & $1.61 \mathrm{E}+04$ & $1.18 \mathrm{E}+02$ & $9.27 \mathrm{E}-07$ & $1.72 \mathrm{E}+00$ & $1.11 \mathrm{E}+01$ \\
$1.00 \mathrm{E}-02$ & $1.61 \mathrm{E}+05$ & $1.18 \mathrm{E}+03$ & $1.28 \mathrm{E}-06$ & $6.64 \mathrm{E}+00$ & $6.29 \mathrm{E}+01$ \\
$1.00 \mathrm{E}-01$ & $1.61 \mathrm{E}+06$ & $1.18 \mathrm{E}+04$ & $1.44 \mathrm{E}-06$ & $1.70 \mathrm{E}+01$ & $2.70 \mathrm{E}+02$ \\
$1.00 \mathrm{E}+00$ & $1.61 \mathrm{E}+07$ & $1.18 \mathrm{E}+05$ & $1.48 \mathrm{E}-06$ & $2.75 \mathrm{E}+01$ & $7.71 \mathrm{E}+02$ \\
\hline
\end{tabular}

${ }^{a}$ Collision frequency in units of $\mathrm{s}^{-1}$

${ }^{b}$ Minimum IERE eigenvalue

${ }^{c} \mathrm{CSE}$ eigenvalues. The number of CSEs equals the number of wells.

\section{Table S6-2. CSE projections for case 6}

\begin{tabular}{cccc}
\hline$p /$ torr & $P_{1}{ }^{a}$ & $P_{2}$ & $P_{3}$ \\
\hline $1.00 \mathrm{E}-08$ & $7.06 \mathrm{E}-07$ & $4.66 \mathrm{E}-02$ & $1.43 \mathrm{E}-01$ \\
$7.00 \mathrm{E}-08$ & $3.80 \mathrm{E}-07$ & $4.40 \mathrm{E}-02$ & $1.42 \mathrm{E}-01$ \\
$1.00 \mathrm{E}-07$ & $3.33 \mathrm{E}-07$ & $4.32 \mathrm{E}-02$ & $1.41 \mathrm{E}-01$ \\
$7.00 \mathrm{E}-07$ & $1.46 \mathrm{E}-07$ & $3.76 \mathrm{E}-02$ & $1.34 \mathrm{E}-01$ \\
$1.00 \mathrm{E}-06$ & $1.23 \mathrm{E}-07$ & $3.63 \mathrm{E}-02$ & $1.32 \mathrm{E}-01$ \\
$5.00 \mathrm{E}-06$ & $5.08 \mathrm{E}-08$ & $2.98 \mathrm{E}-02$ & $1.21 \mathrm{E}-01$ \\
$1.00 \mathrm{E}-05$ & $3.29 \mathrm{E}-08$ & $2.68 \mathrm{E}-02$ & $1.14 \mathrm{E}-01$ \\
$5.00 \mathrm{E}-05$ & $1.04 \mathrm{E}-08$ & $1.94 \mathrm{E}-02$ & $9.74 \mathrm{E}-02$ \\
$1.00 \mathrm{E}-04$ & $5.95 \mathrm{E}-09$ & $1.63 \mathrm{E}-02$ & $8.92 \mathrm{E}-02$ \\
$1.00 \mathrm{E}-03$ & $6.62 \mathrm{E}-10$ & $7.43 \mathrm{E}-03$ & $5.96 \mathrm{E}-02$ \\
$1.00 \mathrm{E}-02$ & $4.10 \mathrm{E}-11$ & $2.17 \mathrm{E}-03$ & $3.10 \mathrm{E}-02$ \\
$1.00 \mathrm{E}-01$ & $1.32 \mathrm{E}-12$ & $3.26 \mathrm{E}-04$ & $1.07 \mathrm{E}-02$ \\
$1.00 \mathrm{E}+00$ & $2.52 \mathrm{E}-14$ & $2.00 \mathrm{E}-05$ & $1.92 \mathrm{E}-03$ \\
\hline
\end{tabular}

${ }^{a} \mathrm{CSE}$ eigenvector projection on the relaxation subspace 
13. Table S7-1. Eigenvalues $\left(s^{-1}\right)$ for case 7

\begin{tabular}{ccccccc}
\hline$p /$ torr & $Z^{a}$ & $R^{b}$ & $E_{1}{ }^{c}$ & $E_{2}$ & $E_{3}$ & $E_{4}$ \\
\hline $1.00 \mathrm{E}-08$ & $1.61 \mathrm{E}-01$ & $1.19 \mathrm{E}-03$ & $1.37 \mathrm{E}-08$ & $2.90 \mathrm{E}-07$ & $3.17 \mathrm{E}-06$ & $7.73 \mathrm{E}-05$ \\
$7.00 \mathrm{E}-08$ & $1.13 \mathrm{E}+00$ & $8.29 \mathrm{E}-03$ & $1.17 \mathrm{E}-08$ & $1.52 \mathrm{E}-06$ & $1.77 \mathrm{E}-05$ & $5.22 \mathrm{E}-04$ \\
$1.00 \mathrm{E}-07$ & $1.61 \mathrm{E}+00$ & $1.18 \mathrm{E}-02$ & $2.07 \mathrm{E}-08$ & $2.05 \mathrm{E}-06$ & $2.41 \mathrm{E}-05$ & $7.36 \mathrm{E}-04$ \\
$7.00 \mathrm{E}-07$ & $1.13 \mathrm{E}+01$ & $8.28 \mathrm{E}-02$ & $3.54 \mathrm{E}-08$ & $9.70 \mathrm{E}-06$ & $1.22 \mathrm{E}-04$ & $4.65 \mathrm{E}-03$ \\
$1.00 \mathrm{E}-06$ & $1.61 \mathrm{E}+01$ & $1.18 \mathrm{E}-01$ & $5.06 \mathrm{E}-08$ & $1.28 \mathrm{E}-05$ & $1.62 \mathrm{E}-04$ & $6.48 \mathrm{E}-03$ \\
$5.00 \mathrm{E}-06$ & $8.06 \mathrm{E}+01$ & $5.91 \mathrm{E}-01$ & $1.06 \mathrm{E}-07$ & $4.15 \mathrm{E}-05$ & $5.54 \mathrm{E}-04$ & $2.79 \mathrm{E}-02$ \\
$1.00 \mathrm{E}-05$ & $1.61 \mathrm{E}+02$ & $1.18 \mathrm{E}+00$ & $2.06 \mathrm{E}-07$ & $6.70 \mathrm{E}-05$ & $9.12 \mathrm{E}-04$ & $5.13 \mathrm{E}-02$ \\
$5.00 \mathrm{E}-05$ & $8.06 \mathrm{E}+02$ & $5.91 \mathrm{E}+00$ & $3.92 \mathrm{E}-07$ & $1.88 \mathrm{E}-04$ & $2.67 \mathrm{E}-03$ & $2.01 \mathrm{E}-01$ \\
$1.00 \mathrm{E}-04$ & $1.61 \mathrm{E}+03$ & $1.18 \mathrm{E}+01$ & $4.97 \mathrm{E}-07$ & $2.83 \mathrm{E}-04$ & $4.06 \mathrm{E}-03$ & $3.54 \mathrm{E}-01$ \\
$1.00 \mathrm{E}-03$ & $1.61 \mathrm{E}+04$ & $1.18 \mathrm{E}+02$ & $8.90 \mathrm{E}-07$ & $9.12 \mathrm{E}-04$ & $1.32 \mathrm{E}-02$ & $2.01 \mathrm{E}+00$ \\
$1.00 \mathrm{E}-02$ & $1.61 \mathrm{E}+05$ & $1.18 \mathrm{E}+03$ & $1.20 \mathrm{E}-06$ & $2.15 \mathrm{E}-03$ & $3.00 \mathrm{E}-02$ & $8.66 \mathrm{E}+00$ \\
$1.00 \mathrm{E}-01$ & $1.61 \mathrm{E}+06$ & $1.17 \mathrm{E}+04$ & $1.34 \mathrm{E}-06$ & $3.67 \mathrm{E}-03$ & $4.76 \mathrm{E}-02$ & $2.55 \mathrm{E}+01$ \\
$1.00 \mathrm{E}+00$ & $1.61 \mathrm{E}+07$ & $1.17 \mathrm{E}+05$ & $1.40 \mathrm{E}-06$ & $4.76 \mathrm{E}-03$ & $5.78 \mathrm{E}-02$ & $4.77 \mathrm{E}+01$ \\
\hline
\end{tabular}

${ }^{a}$ Collision frequency in units of $\mathrm{s}^{-1}$

${ }^{b}$ Minimum IERE eigenvalue

${ }^{c} \mathrm{CSE}$ eigenvalues. The number of CSEs equals the number of wells.

\section{Table S7-2. CSE projections for case 7}

\begin{tabular}{ccccc}
\hline$p$ torr & $P_{1}{ }^{a}$ & $P_{2}$ & $P_{3}$ & $P_{4}$ \\
\hline $1.00 \mathrm{E}-08$ & $7.09 \mathrm{E}-07$ & $9.61 \mathrm{E}-05$ & $1.31 \mathrm{E}-03$ & $4.42 \mathrm{E}-02$ \\
$7.00 \mathrm{E}-08$ & $3.80 \mathrm{E}-07$ & $7.00 \mathrm{E}-05$ & $1.01 \mathrm{E}-03$ & $4.24 \mathrm{E}-02$ \\
$1.00 \mathrm{E}-07$ & $3.33 \mathrm{E}-07$ & $6.54 \mathrm{E}-05$ & $9.57 \mathrm{E}-04$ & $4.19 \mathrm{E}-02$ \\
$7.00 \mathrm{E}-07$ & $1.45 \mathrm{E}-07$ & $4.19 \mathrm{E}-05$ & $6.51 \mathrm{E}-04$ & $3.74 \mathrm{E}-02$ \\
$1.00 \mathrm{E}-06$ & $1.22 \mathrm{E}-07$ & $3.81 \mathrm{E}-05$ & $5.97 \mathrm{E}-04$ & $3.64 \mathrm{E}-02$ \\
$5.00 \mathrm{E}-06$ & $5.02 \mathrm{E}-08$ & $2.31 \mathrm{E}-05$ & $3.80 \mathrm{E}-04$ & $3.07 \mathrm{E}-02$ \\
$1.00 \mathrm{E}-05$ & $3.24 \mathrm{E}-08$ & $1.80 \mathrm{E}-05$ & $3.00 \mathrm{E}-04$ & $2.80 \mathrm{E}-02$ \\
$5.00 \mathrm{E}-05$ & $1.01 \mathrm{E}-08$ & $9.13 \mathrm{E}-06$ & $1.55 \mathrm{E}-04$ & $2.11 \mathrm{E}-02$ \\
$1.00 \mathrm{E}-04$ & $5.72 \mathrm{E}-09$ & $6.49 \mathrm{E}-06$ & $1.11 \mathrm{E}-04$ & $1.82 \mathrm{E}-02$ \\
$1.00 \mathrm{E}-03$ & $6.07 \mathrm{E}-10$ & $1.64 \mathrm{E}-06$ & $2.66 \mathrm{E}-05$ & $9.17 \mathrm{E}-03$ \\
$1.00 \mathrm{E}-02$ & $3.58 \mathrm{E}-11$ & $2.63 \mathrm{E}-07$ & $3.73 \mathrm{E}-06$ & $3.14 \mathrm{E}-03$ \\
$1.00 \mathrm{E}-01$ & $1.48 \mathrm{E}-12$ & $2.44 \mathrm{E}-08$ & $2.82 \mathrm{E}-07$ & $5.98 \mathrm{E}-04$ \\
$1.00 \mathrm{E}+00$ & $6.51 \mathrm{E}-14$ & $1.31 \mathrm{E}-09$ & $1.14 \mathrm{E}-08$ & $5.03 \mathrm{E}-05$ \\
\hline
\end{tabular}

${ }^{a} \mathrm{CSE}$ eigenvector projection on the relaxation subspace 


\section{Convergence checks and example of variation of $\langle\Delta E\rangle_{\text {down }}$}

Convergence checks. Here we present some convergence checks with respect to numerical parameters of the MESS program. The results are shown in Fig. S1.

ExponenCutOff is a numerical parameter used in implementing the energy transfer model. The code ignores any energy transfer that would have an excitation probability below a given threshold (specified as $\exp (-$ ExponenCutoff)). The default value for this is ExponenCutoff $=10$, which is what we sued in the calculations reported in the article proper. In cases $3 \mathrm{~A}-3 \mathrm{E}$, we show that the results are well converged with respect to using larger values.

ExcessEnergyOverTemperature is a numerical parameter that determines the maximum energy used in master equation energy bins. In case $3 \mathrm{E}$, we show good convergence with respect to this parameter.

EnergyStepOverTemperature is a numerical parameter that determines the energy step of master equation energy bins. In case $3 \mathrm{E}$, we show good convergence with respect to this parameter.

Variation of energy transfer parameter. Figure $\mathrm{S} 1$ demonstrates the fact of changing the $\langle\Delta E\rangle_{\text {down }}$ parameter.

\section{Case 3:}

Edown 130

ExponenCutOff 10

ExcessEnergyOverTemperature 30

EnergyStepOverTemperature 0.2

Case 3A:

Edown 130

ExponenCutOff 50

ExcessEnergyOverTemperature 30

EnergyStepOverTemperature 0.2

Case 3B:

Edown 130

ExponenCutOff 70

ExcessEnergyOverTemperature 30

EnergyStepOverTemperature 0.2

Case 3C:

Edown 130

ExponenCutOff 100

ExcessEnergyOverTemperature 30

EnergyStepOverTemperature $\quad 0.2$

Case 3D:

Edown 130

ExponenCutOff 150

ExcessEnergyOverTemperature 30

EnergyStepOverTemperature 0.2

Case 3E:

Edown 130

ExponenCutOff 150

ExcessEnergyOverTemperature 40

EnergyStepOverTemperature $\quad 0.1$

Case 3F:

Edown 60

ExponenCutOff 10 


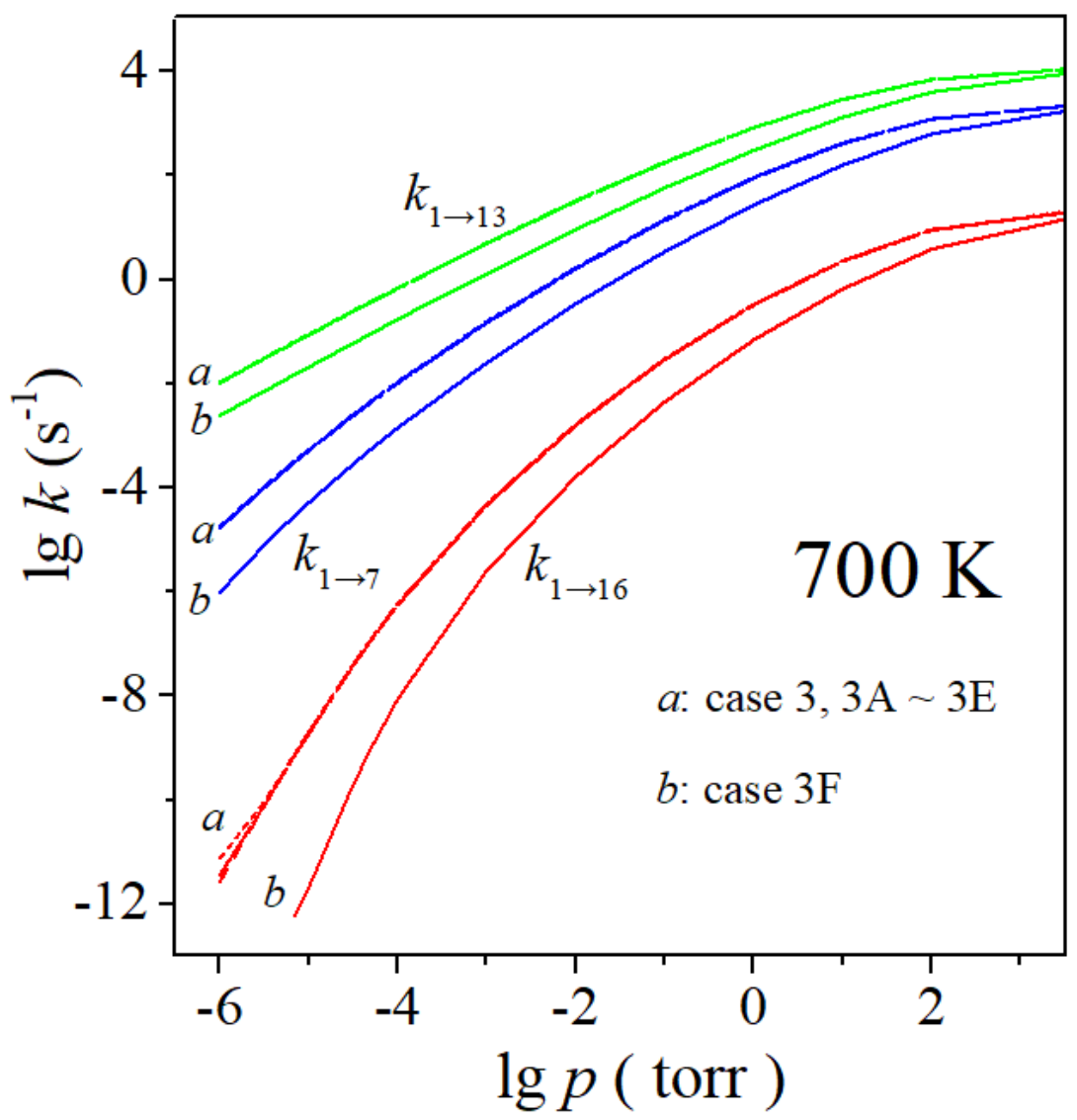

Figure S1. Convergence checks and an example of variation of $\langle\Delta E\rangle_{\text {down }}$. To within plotting accuracy, the results for cases $3 \mathrm{~A}-3 \mathrm{E}$ are indistinguishable from those for case 3 . This shows good convergence with respect to numerical parameters. The rate constants in case $3 \mathrm{~F}$, which corresponds to decreasing the strength of energy transfer in the physical system, are smaller, but the low-pressure power behavior is similar to that for case 3 . 


\section{Additional enthalpy profiles}
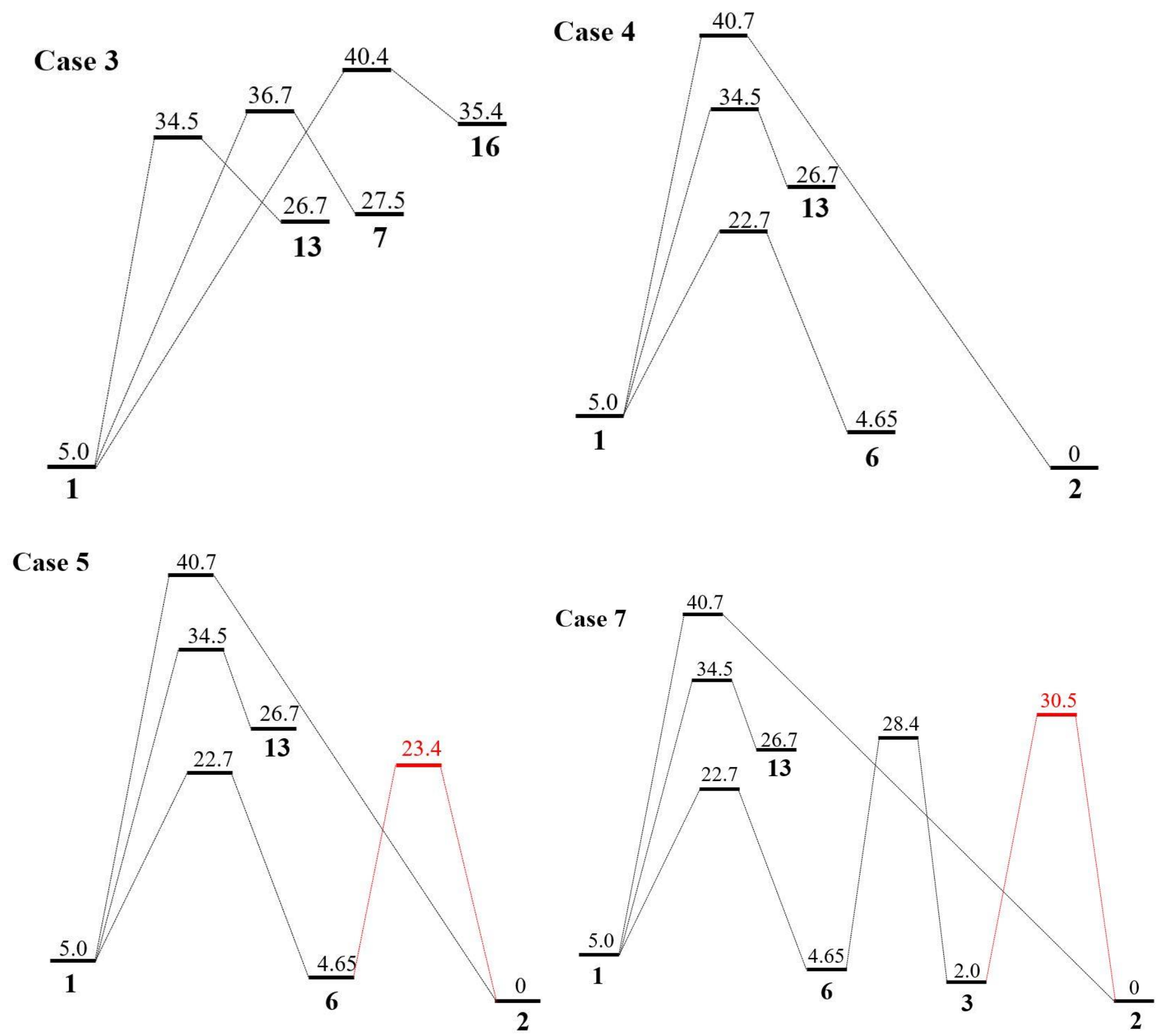

Figure S2. Enthalpy profile of stationary points for cases 3, 4, 5, and 7. Numbers above the line denote enthalpy or enthalpy of activation at $0 \mathrm{~K}$ relative to isomer 2 in units of $\mathrm{kcal} / \mathrm{mol}$. 


\section{Note on quadruple precision}

A numerical issue should be mentioned. When the threshold is high and the pressure is low, the range of magnitudes of the values of the distributions may vary to such a great extent that the calculation of the eigenvectors of the transition matrix requires quadruple precision. To illustrate this we define the summand in eq 5 as

$$
\delta k(E)=\hat{k}_{1 \rightarrow \mathrm{X}}(E) g(E)
$$

and Fig. S1 presents the calculated $\delta k(E)$ for case 3 as calculated in both double and quadruple precision. One can see that double precision is insufficient at high energy. We have checked all plots in this work by quadruple precision. We present this example to warn other researchers to be careful in applying the master equation at low pressure.
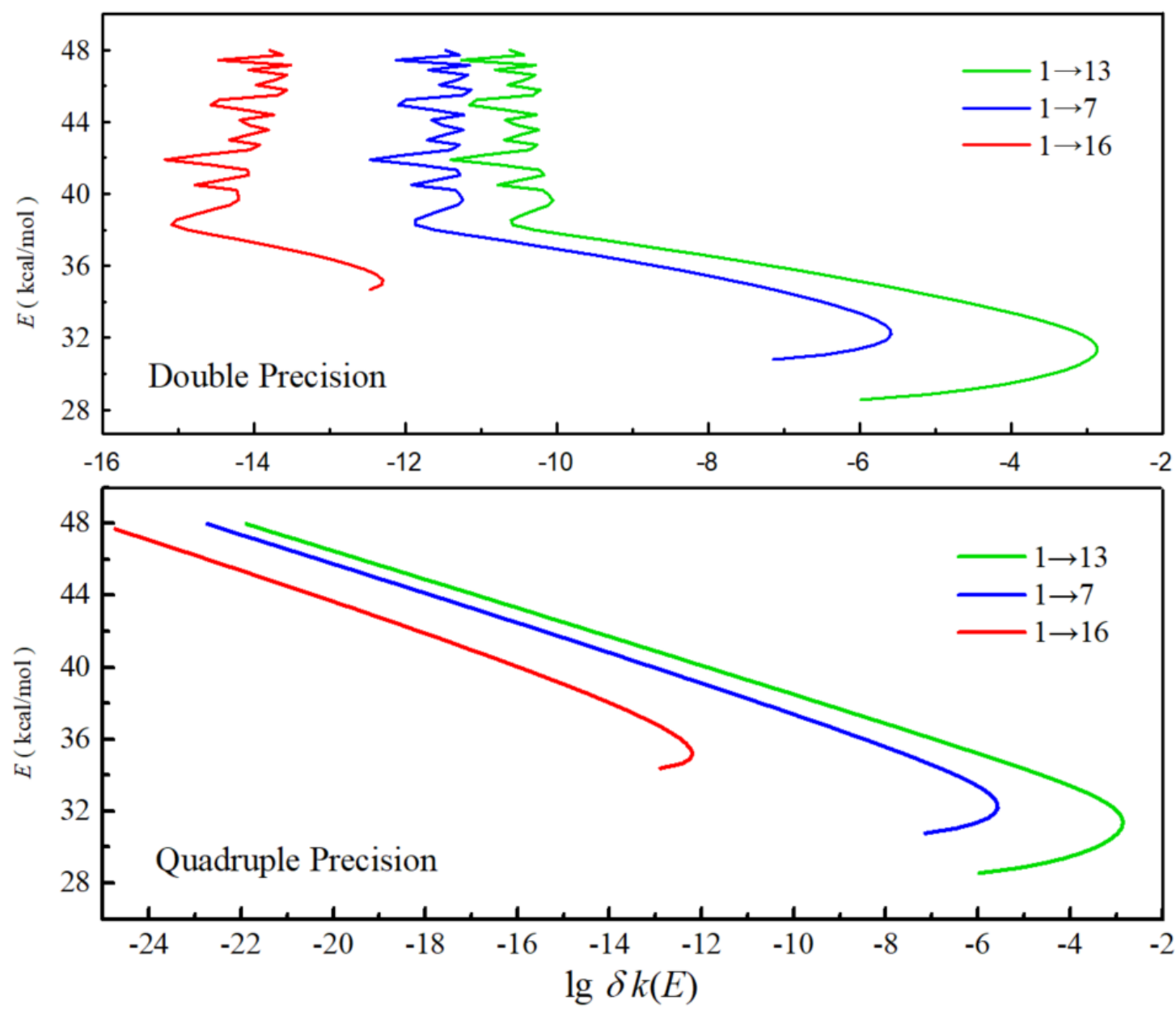

Figure S3. Comparison of double and quadruple precision for calculating $\delta k(E)$ for case 3 at a temperature of $700 \mathrm{~K}$ and a pressure of $1 \times 10^{-6}$ torr. 


\section{Input file for MESS for case 1 and case 2}

TemperatureList [K]

Pressurelist [torr]

EnergystepoverTemperature

ExcessEnergyOverTemperature

ModelEnergyLimit [ $\mathrm{kcal} / \mathrm{mol}]$

CalculationMethod

ChemicalEigenvalueMax

ChemicalEigenvalueMin

EigenvalueOutput

Model

EnergyRelaxation

Exponential

Factor $[1 / \mathrm{cm}]$

Power

End

CollisionFrequency

LennardJones Epsilons [1/cm] Sigmas [angstrom] End Masses [amu ]

$\begin{array}{ll}\text { Well } & 1 \\ & \text { Species } \\ & \text { RRHO }\end{array}$

400. 1000 .

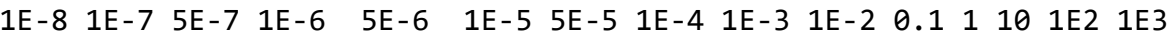

.2

30

400

direct

.2

eval.out

$1 \mathrm{E}-7$

130.

0

124.342197 .807

3.6557 .61

4099 ! Ar

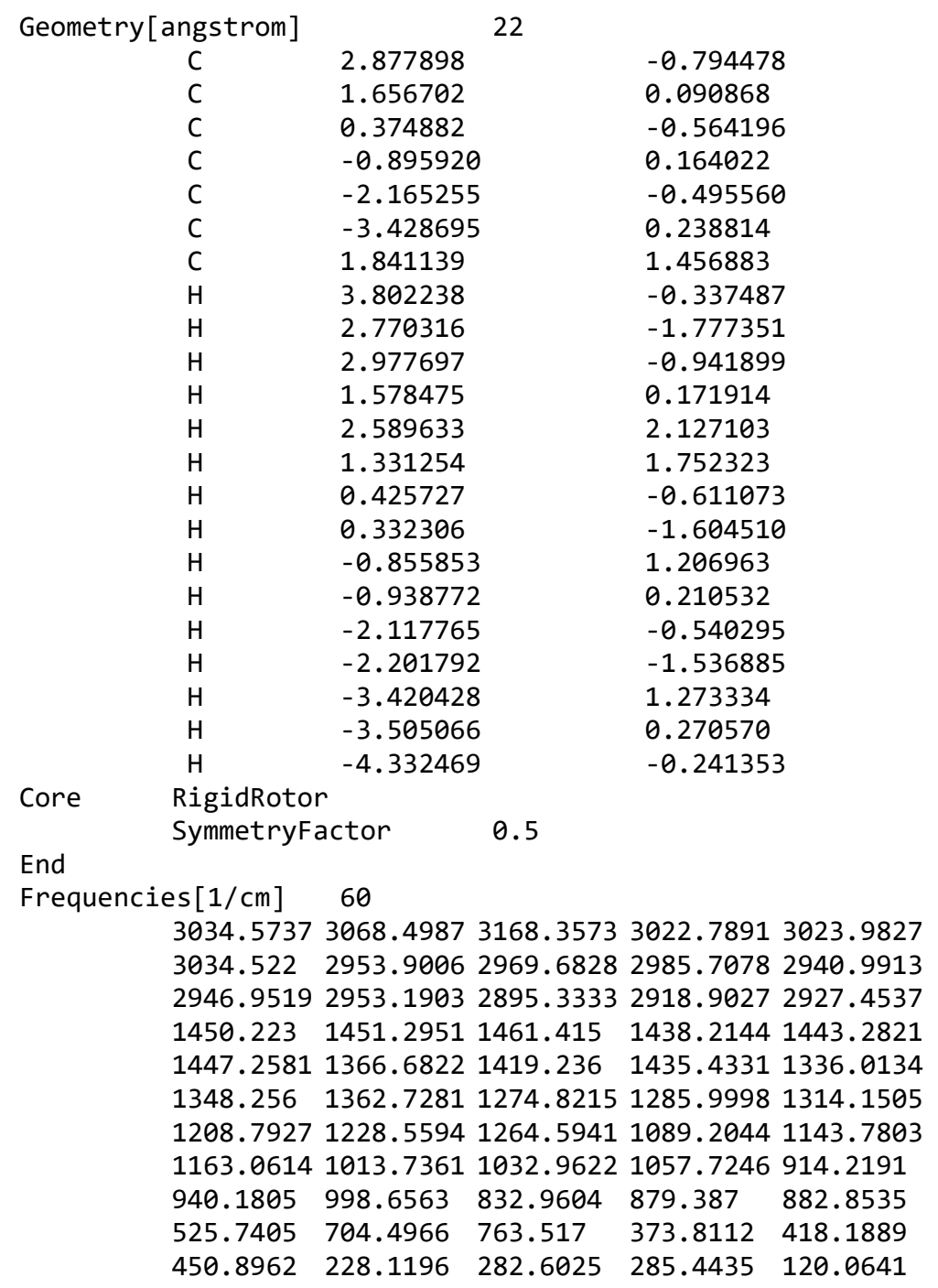

0.027662

0.333186

$-0.186344$

0.235953

$-0.292788$

0.141314

$-0.232058$

0.391760

0.498471

$-1.052897$

1.429911

0.172026

$-1.142041$

$-1.284847$

0.163433

$-0.107692$

1.333670

$-1.388538$

0.052687

$-0.217358$

1.233171

$-0.243309$ 
$\begin{array}{lllll}154.2477 & 223.7787 & 55.6091 & 75.2174 & 92.5654\end{array}$

End

ZeroEnergy [ $\mathrm{kcal} / \mathrm{mol}]$

$-19.92656417248466$

Well 2

Species

RRHO

Geometry[angstrom]

H 4.275307

C $\quad 3.289217$

C $\quad 2.165331$

$\mathrm{H} \quad 3.226186$

$\mathrm{H} \quad 3.227725$

C $\quad 0.783315$

$\mathrm{H} \quad 2.258573$

$\mathrm{H} \quad 2.255390$

C $\quad-0.356615$

$\mathrm{H} \quad 0.684922$

$\mathrm{H} \quad-0.241477$

$\mathrm{H} \quad 0.678855$

C $\quad-1.709530$

C $\quad-2.228424$

C $\quad-2.250976$

$\mathrm{H} \quad-0.237608$

$\mathrm{H} \quad-1.825598$

$\mathrm{H} \quad-1.953315$

H $\quad-3.319199$

$\mathrm{H} \quad-2.002046$

$\mathrm{H} \quad-1.841753$

$\mathrm{H} \quad-3.339863$

\section{Core RigidRotor}

SymmetryFactor $\quad 1.0$

End

Frequencies [1/cm ] 60

3018.47143022 .64163031 .63082969 .30312986 .0646 3017.12752952 .93522967 .98932969 .08112927 .9286 $\begin{array}{llllll}2941.0188 & 2951.669 & 2870.037 & 2873.8071 & 2916.3279\end{array}$ 1447.50511451 .48621459 .49261434 .82181436 .515 $1447.346 \quad 1418.96131421 .96821430 .22511358 .0463$ 1364.63241367 .79831304 .77471311 .52271342 .1663 $1248.332 \quad 1264.74611281 .72231092 .41361189 .1493$ 1217.90361020 .67231042 .71821045 .6843955 .8919

$\begin{array}{llllll}970.4496 & 988.521 & 876.4106 & 891.4236 & 910.4481\end{array}$ $\begin{array}{llllll}704.2294 & 761.8591 & 769.6757 & 373.2334 & 395.3717\end{array}$ $\begin{array}{lllll}455.5774 & 228.3355 & 238.22 & 315.7114 & 111.0644\end{array}$ $\begin{array}{lllll}119.9266 & 132.9308 & 34.5805 & 79.8316 & 103.8847\end{array}$

End ZeroEnergy [kcal/mol] $-24.902086997978437$

End

Well 3

Species RRHO

$-0.077987$

$-0.550075$

0.479556

$-1.220911$

$-1.168118$

$-0.163744$

1.159347

1.105759

0.870971

$-0.789977$

1.492884

$-0.839185$

0.241551

$-0.291512$

$-0.322016$

1.536912

$-1.295319$

0.353123

$-0.393225$

0.309023

$-1.325478$

$-0.435225$ 


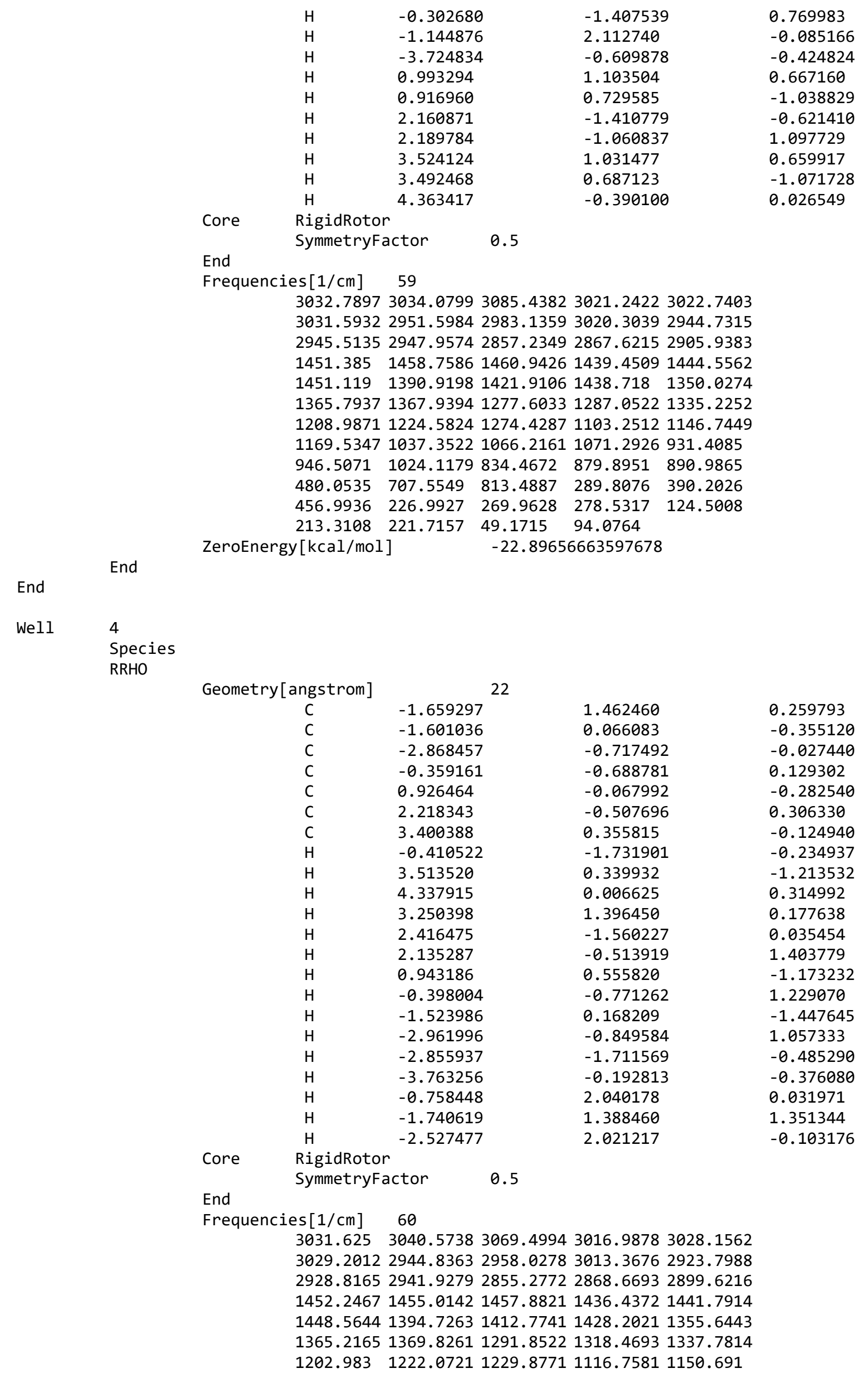


1163.30891027 .46971056 .45461084 .3282934 .2896

$\begin{array}{lllll}945.4581 & 1013.1063 & 840.2219 & 890.7771 & 895.5109\end{array}$

$\begin{array}{llllll}465.7549 & 738.8034 & 808.1897 & 361.144 & 389.4378\end{array}$

$\begin{array}{llllll}416.3728 & 241.6431 & 281.8537 & 298.3786 & 146.7782\end{array}$

$\begin{array}{lllll}215.3726 & 222.4213 & 33.1775 & 45.9328 & 104.594\end{array}$

ZeroEnergy [kcal/mol] $\quad-23.549176515969908$

End

End

Well 5

Species

RRHO

\begin{tabular}{clll} 
Geometry[angstrom] & \multicolumn{2}{c}{22} & 0.193133 \\
C & -2.221379 & 0.486787 & -0.158774 \\
C & -0.959782 & -0.215771 & 0.164704 \\
C & 0.295228 & 0.591357 & -0.076375 \\
C & -3.535370 & -0.149033 & -0.356531 \\
C & 1.596585 & -0.021332 & -0.028040 \\
C & 2.775036 & 0.892127 & 0.214624 \\
C & 1.835175 & -1.418129 & -1.232992 \\
H & -0.963578 & -0.473055 & 1.254933 \\
H & 0.374058 & 0.718031 & -0.253767 \\
H & 0.183786 & 1.600667 & 0.553776 \\
H & -3.695175 & -1.038760 & -1.115346 \\
H & -3.602048 & -0.500105 & 0.103957 \\
H & -4.370246 & 0.531751 & -1.450592 \\
H & 1.514812 & -0.104320 & 0.359512 \\
H & -0.922568 & -1.189671 & 0.763923 \\
H & -2.169550 & 1.406846 & 1.058640 \\
H & 2.892398 & 0.982710 & -0.432495 \\
H & 2.626364 & 1.898300 & -0.431871 \\
H & 3.712252 & 0.496983 & -0.097609 \\
H & 1.065536 & -2.129424 & 1.311378 \\
H & 1.837162 & -1.383367 & -0.107884 \\
H & 2.803838 & -1.812630 &
\end{tabular}

$\begin{array}{ll}\text { Core } & \text { RigidRotor } \\ & \text { SymmetryFactor } \quad 0.5\end{array}$

End

0.5

Frequencies $[1 / \mathrm{cm}] \quad 60$

3032.11483033 .13833109 .67033013 .66563016 .9981

3027.94642944 .40292949 .20832970 .74432917 .4729

$2926.86752941 .652 \quad 2871.42762890 .9372 \quad 2895.414$

1445.55311453 .91081462 .25231434 .51531440 .6427

1441.77881387 .42411420 .02921426 .19631356 .1836

$1363.22781371 .86781290 .09441324 .091 \quad 1338.8925$

$1189.763 \quad 1227.30881264 .618 \quad 1116.41281148 .9117$

1163.62911037 .56381062 .79091072 .6737937 .6449

$\begin{array}{lllll}947.743 & 962.6424 & 890.2454 & 894.747 & 902.6924\end{array}$

$\begin{array}{llllll}459.2119 & 724.1804 & 814.0155 & 303.0659 & 390.8973\end{array}$

$\begin{array}{lllll}422.5765 & 241.3106 & 280.3909 & 290.9602 & 101.2684\end{array}$

$\begin{array}{lllll}129.4542 & 216.1213 & 21.9736 & 73.8136 & 88.1011\end{array}$

ZeroEnergy [ $\mathrm{kcal} / \mathrm{mol}]$

$-23.43685231550381$

End

End

Well 6

Species

RRHO

Geometry[angstrom]
C
C
C
C
H
H
C
H

-1.570543
-0.263497
0.987746
-2.746698
-0.163361
-0.332205
2.274107
0.970629

22

0.024556

$-0.583827$

0.214357

$-0.884701$

$-1.601148$

$-0.696195$

$-0.515603$

1.187031

-0.357283
0.156491
-0.192748
-0.009564
-0.247578
1.249624
0.191244
0.315006 


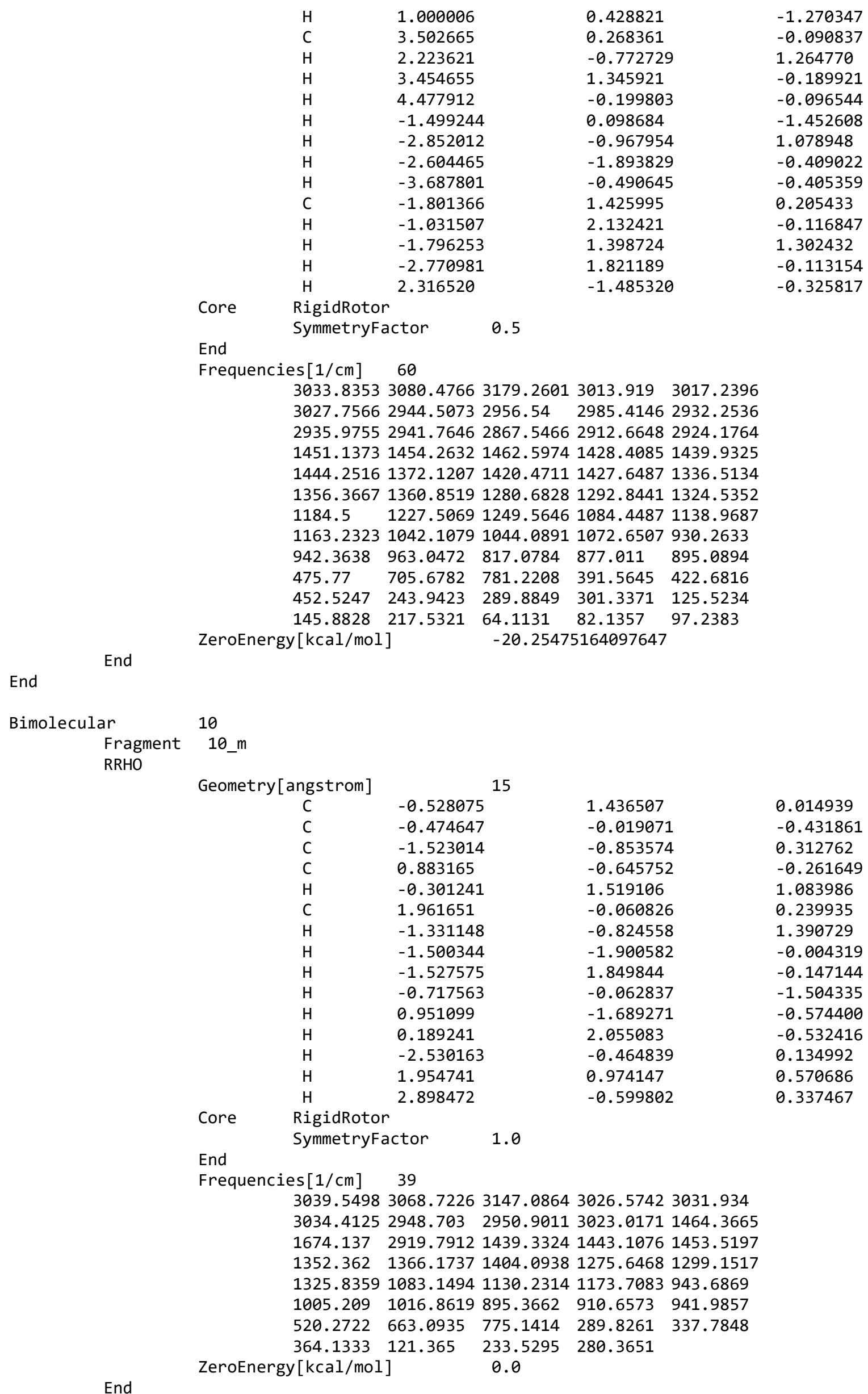


Fragment 10_r

RRHO

End

\begin{tabular}{|c|c|c|c|}
\hline Geometry[angstrom] & \multicolumn{2}{|r|}{7} & \\
\hline C & 0.009068 & & -0.691615 \\
\hline $\mathrm{H}$ & -1.013062 & & -1.101730 \\
\hline $\mathrm{H}$ & 0.506467 & & -1.097666 \\
\hline $\mathrm{H}$ & 0.506467 & & -1.097666 \\
\hline C & 0.009068 & & 0.792013 \\
\hline $\mathrm{H}$ & -0.054342 & & 1.347336 \\
\hline $\mathrm{H}$ & -0.054342 & & 1.347336 \\
\hline \multicolumn{3}{|l|}{ RigidRotor } & \\
\hline \multicolumn{2}{|c|}{ SymmetryFactor } & 1.0 & \\
\hline \multicolumn{4}{|l|}{ End } \\
\hline Frequencies $[1 / \mathrm{cm}]$ & 15 & & \\
\hline 3029.463 & 3088.5523 & 3185.6108 & 1438.82842900 .9511 \\
\hline 2984.4146 & 1358.1354 & 1423.0373 & 1438.2138950 .5832 \\
\hline $\begin{array}{r}1051.8124 \\
\text { ZeroEnergy[kcal/mol }\end{array}$ & 1158.077 & $\begin{array}{l}110.9322 \\
0.0\end{array}$ & 472.0731786 .3139 \\
\hline
\end{tabular}

End

GroundEnergy [ $\mathrm{kcal} / \mathrm{mol}] \quad 0.0$

Bimolecular 11

Fragment 11_m

RRHO

Geometry[angstrom]

C $\quad-0.000069$

12

C $\quad 0.000029 \quad 0.125243$

0.000000

0.000000

$-0.885808$

0.885808

0.000000

$-0.925820$

0.925820

\begin{tabular}{|c|c|c|c|c|c|}
\hline & Geometry[angstrom] & & 12 & & \\
\hline & C & -0.000069 & & 1.453035 & 0.000000 \\
\hline & $\mathrm{H}$ & 0.000345 & & 2.018204 & 0.927678 \\
\hline & C & 0.000029 & & 0.125243 & 0.000000 \\
\hline & C & -0.000069 & & -0.676503 & -1.269640 \\
\hline & C & -0.000069 & & -0.676503 & 1.269640 \\
\hline & $\mathrm{H}$ & 0.000345 & & 2.018204 & -0.927678 \\
\hline & $\mathrm{H}$ & 0.879224 & & -1.329796 & -1.313290 \\
\hline & $\mathrm{H}$ & 0.000357 & & -0.034663 & -2.153088 \\
\hline & $\mathrm{H}$ & -0.879388 & & -1.329559 & -1.313573 \\
\hline & $\mathrm{H}$ & 0.000357 & & -0.034663 & 2.153088 \\
\hline & $\mathrm{H}$ & 0.879224 & & -1.329796 & 1.313290 \\
\hline & $\mathrm{H}$ & -0.879388 & & -1.329559 & 1.313573 \\
\hline & RigidRotor & & & & \\
\hline & SymmetryF & actor & 2.0 & & \\
\hline & End & & & & \\
\hline & Frequencies [1/cm ] & 30 & & & \\
\hline & 3045.3897 & 3061.7783 & 3144.3665 & 3000.99743002 .1008 & \\
\hline & 3044.0586 & 1694.9501 & 2942.5339 & $2945.723 \quad 1433.1765$ & \\
\hline & 1439.8295 & 1449.4695 & 1369.119 & 1394.24561421 .7726 & \\
\hline & 1077.8208 & 1268.6951 & 1362.3041 & 989.588 & \\
\hline & 1050.6289 & 807.418 & 914.295 & $933.6194 \quad 420.8622$ & \\
\hline & 426.5021 & 689.1107 & 169.3417 & $208.7868 \quad 361.3454$ & \\
\hline & ZeroEnergy [kcal/mol & & 0.0 & & \\
\hline End & & & & & \\
\hline Fragment & 11_r & & & & \\
\hline & Geometry[angstrom] & & 10 & & \\
\hline & $\mathrm{C}$ & 1.216289 & & -0.241742 & -0.023403 \\
\hline & $\mathrm{H}$ & 1.277212 & & -0.817365 & -0.951700 \\
\hline & $\mathrm{H}$ & 2.094549 & & 0.406255 & 0.032261 \\
\hline & $\mathrm{H}$ & 1.266801 & & -0.949732 & 0.809815 \\
\hline & C & -0.081295 & & 0.560866 & 0.031130 \\
\hline & $\mathrm{H}$ & -0.091647 & & 1.171445 & 0.949681 \\
\hline & $\mathrm{H}$ & -0.106750 & & 1.291075 & -0.789694 \\
\hline & C & -1.291996 & & -0.297866 & -0.019525 \\
\hline & $\mathrm{H}$ & -1.223856 & & -1.357147 & 0.195978 \\
\hline & $\mathrm{H}$ & -2.274299 & & 0.127918 & -0.175559 \\
\hline & RigidRotor & & & & \\
\hline & SymmetryF & actor & 1.0 & & \\
\hline & $\begin{array}{l}\text { End } \\
\text { Frequencies }[1 / \mathrm{cm}]\end{array}$ & 24 & & & \\
\hline
\end{tabular}


End

ZeroEnergy $[\mathrm{kcal} / \mathrm{mol}] \quad 0.0$

3039.67863079 .31623178 .61612938 .74442957 .3185

3028.5391449 .25891456 .50352885 .61471364 .4066

1423.27161425 .06521139 .66671229 .72291321 .8726

$900.7013 \quad 1017.4751 \quad 1069.7662463 .7675 \quad 733.9277$

$\begin{array}{llll}866.5687 & 79.5984 & 237.2419 & 360.4634\end{array}$

GroundEnergy [kcal/mol]

$-2.5740439689930645$

End

12

Bimolecular

Fragment $12 \mathrm{~m}$

RRHO

Geometry[angstrom]

C $\quad 1.846946$

12

C $\quad 0.715098$

C $\quad-0.536468$

$-1.714370$

2.720807

$-1.904284$

$-2.629837$

$-1.505977$

$-0.791192$

$-0.353066$

C

0.658182

1.938130

0.015781

$-0.296376$

0.527831

$-0.248877$

$-0.625582$

$-1.163115$

0.348316

$-0.540920$

0.844468

1.440410

$-1.226837$

0.933111

1.0

End

RigidRotor

SymmetryFactor

Frequencies $[1 / \mathrm{cm}] \quad 30$

3040.60493055 .61053142 .57262989 .23763031 .43

$3039.37871681 .23042943 .731 \quad 2958.23591434 .3926$

1448.07781456 .56061303 .88921361 .37731406 .6301

1165.89331249 .79691277 .86561001 .96441016 .6186

$\begin{array}{lllll}1066.8964 & 842.867 & 941.5012 & 957.5511 & 421.3843\end{array}$

$\begin{array}{lllll}640.9766 & 766.257 & 103.827 & 217.9229 & 303.5823\end{array}$

ZeroEnergy [kcal/mol]

0.0

End

Fragment 12_r

RRHO

Geometry[angstrom]

C -1.285360

10

H $\quad-2.143926$

$\mathrm{H} \quad-1.296570$

$\mathrm{H}-1.451643$

C $\quad-0.000003$

C $\quad 1.285359$

$\mathrm{H} \quad 1.296587$

$\mathrm{H} \quad 2.143920$

$\mathrm{H} \quad 1.451645$

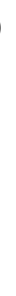

0.000010

RigidRotor

Core

SymmetryFactor

2.0

End

Frequencies $[1 / \mathrm{cm}] \quad 24$

3030.06963030 .81723122 .81072892 .75412953 .2417

2954.84491431 .53261445 .42862889 .93511377 .341

1420.24341430 .69731147 .19021325 .33471368 .7524

$\begin{array}{llllll}920.2626 & 993.5311 & 1125.5459 & 363.8137 & 879.0788\end{array}$

$\begin{array}{llll}905.8628 & 87.3278 & 93.9817 & 317.0982\end{array}$

ZeroEnergy [kcal/mol]

0.0

End

End

GroundEnergy [kcal/mol]

$-1.5606161264834995$

Bimolecular 13

Fragment 13_m 
RRHO

End

Geometry[angstrom]

$\begin{array}{ll}\mathrm{C} & 1.227078 \\ \mathrm{H} & 1.800272 \\ \mathrm{H} & 1.801086 \\ \mathrm{H} & 1.162752 \\ \mathrm{C} & -0.135226 \\ \mathrm{H} & -0.173468 \\ \mathrm{C} & -1.271722 \\ \mathrm{H} & -2.235213 \\ \mathrm{H} & -1.276207\end{array}$

9

$\begin{array}{ll}\text { Core } & \text { RigidRotor } \\ & \text { SymmetryFactor } \quad 1.0\end{array}$

End SymmetryFactor $\quad 1.0$

Frequencies [1/cm ] 21 $\begin{array}{lllll}3054.8273 & 3063.0255 & 3145.1153 & 2953.685 & 3014.79\end{array}$ 3038.46721432 .52831445 .10041680 .74971285 .0092 $1361.18761402 .9944998 .6095 \quad 1045.34991156 .6318$ $\begin{array}{lllll}909.3304 & 913.4013 & 937.4509 & 199.9229 & 410.9365\end{array}$ 576.2202

ZeroEnergy $[\mathrm{kcal} / \mathrm{mol}]$

0.0

Fragment 13_r

RRHO

Geometry[angstrom]

C $\quad 1.525216$

13

-0.161999
0.148233
0.150329
-1.253068
0.458027
1.547313
-0.222539
0.276517
-1.310257

0.000117

$-0.879810$

0.878859

0.001438

$-0.000447$

0.000845

$-0.000082$

0.000960

0.000182

\subsection{2}

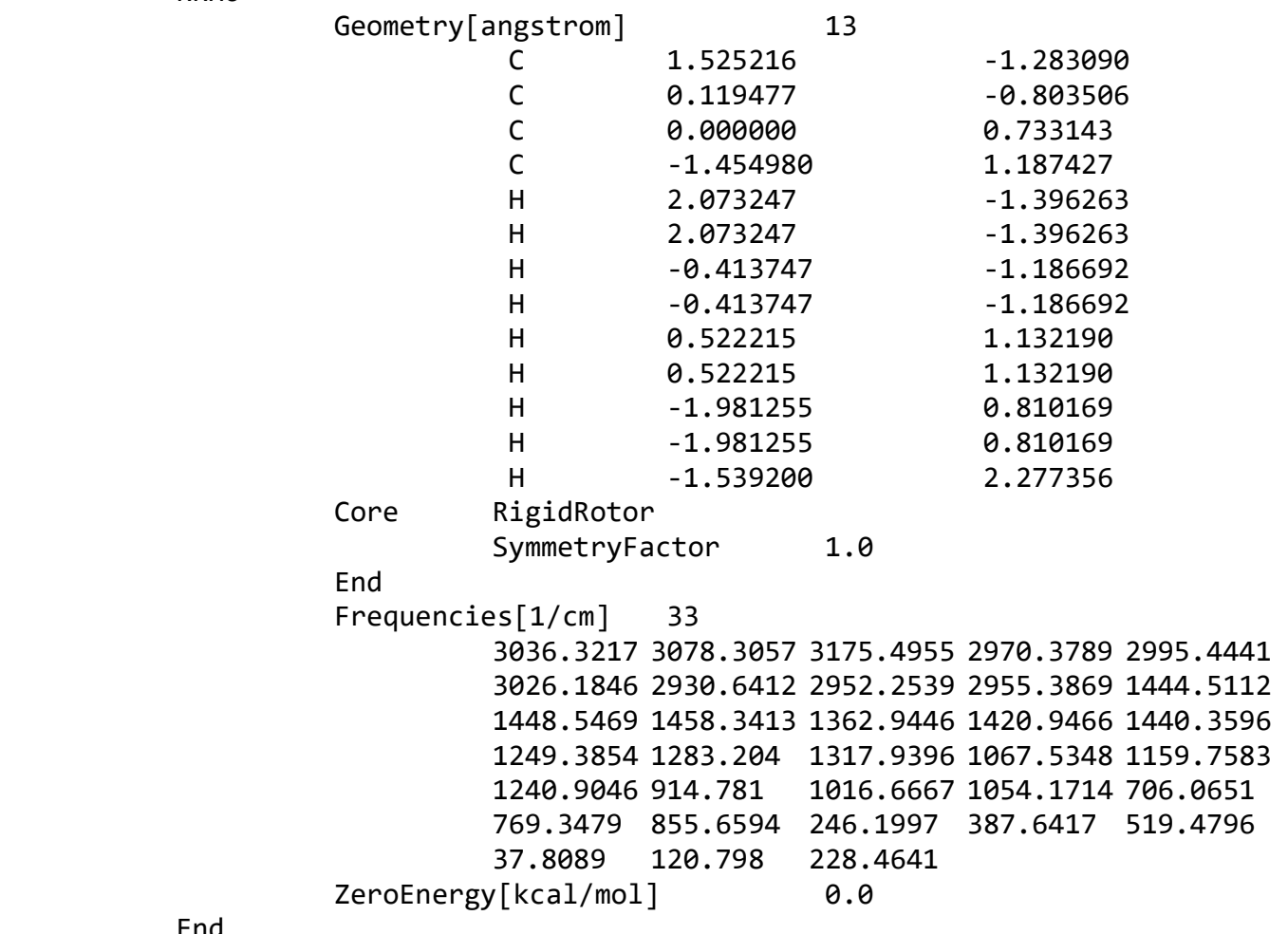

End

GroundEnergy [kcal/mol] $\quad 1.7676942614957243$

$\begin{array}{cc}\text { Bimolecular } & 14 \\ \text { Fragment } & 14 \_m\end{array}$
9

$\begin{array}{ll}\mathrm{C} & -1.227042 \\ \mathrm{H} & -1.800449 \\ \mathrm{H} & -1.162529 \\ \mathrm{H} & -1.800958 \\ \mathrm{C} & 0.135229 \\ \mathrm{H} & 0.173446 \\ \mathrm{C} & 1.271686 \\ \mathrm{H} & 2.235235 \\ \mathrm{H} & 1.276011\end{array}$

0.000000

0.000000

0.000000

0.000000

0.926438

$-0.926438$

0.880040

$-0.880040$

0.877378

$-0.877378$

0.882981

$-0.882981$

0.000000 


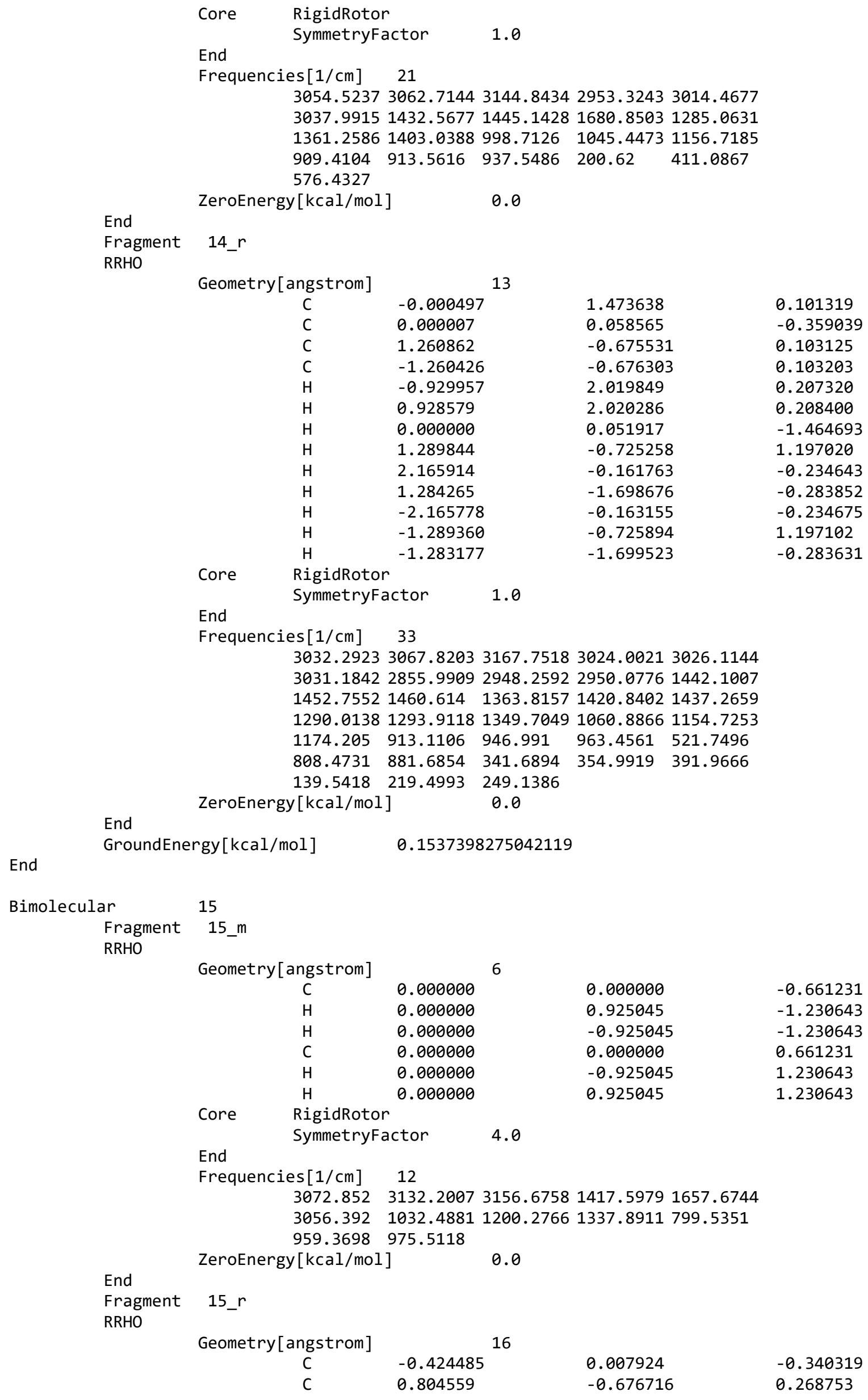




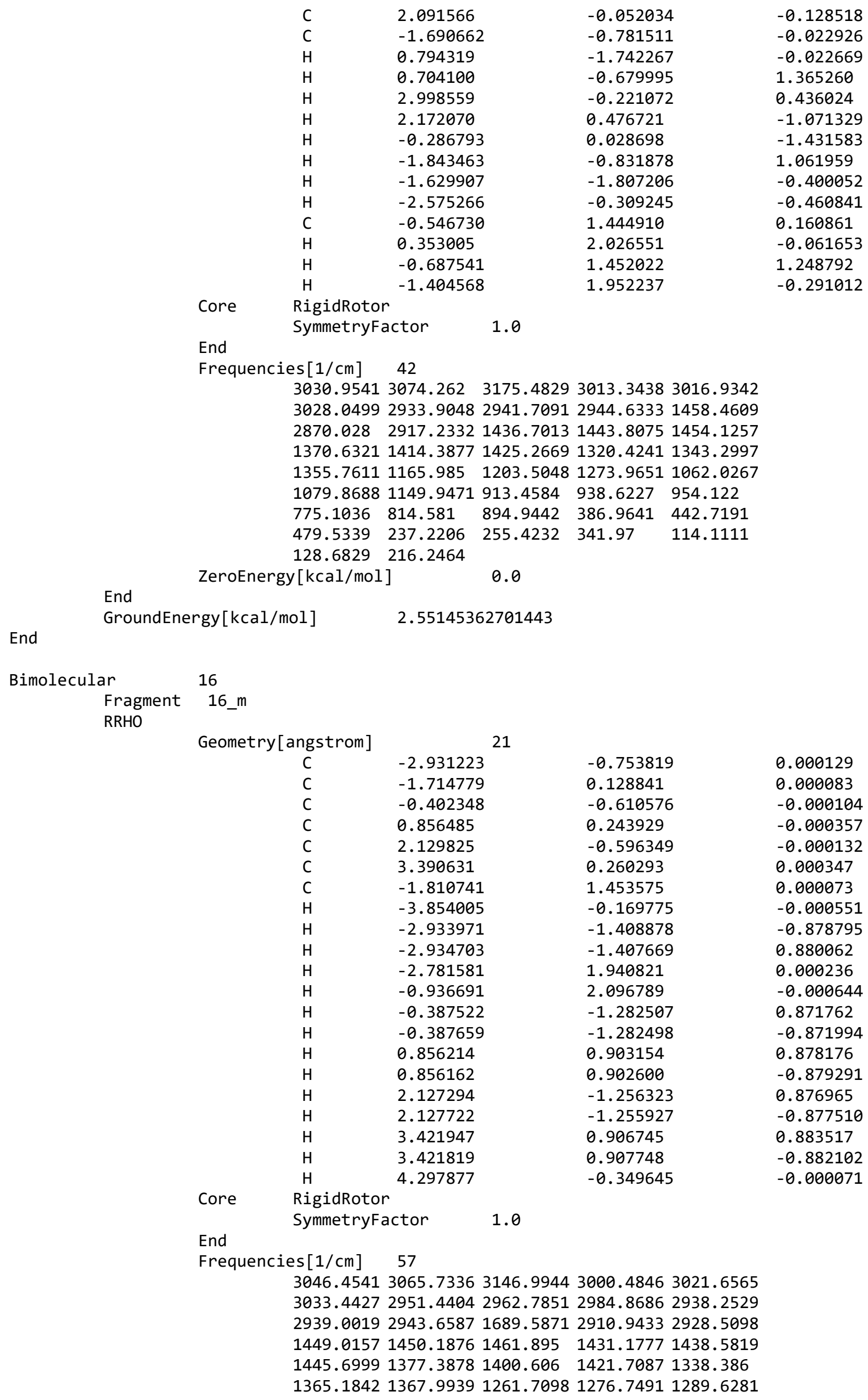


End

$1111.316 \quad 1207.08191209 .04641027 .51921059 .8144$

$\begin{array}{lllll}1106.1909 & 947.1942 & 984.494 & 1022.0829 & 890.1342\end{array}$

$\begin{array}{llllll}908.7654 & 929.0739 & 721.1203 & 782.004 & 836.25\end{array}$

$\begin{array}{llllll}440.6988 & 490.0332 & 675.0396 & 295.5487 & 316.6903\end{array}$

$\begin{array}{llllll}428.4111 & 140.044 & 182.4688 & 238.6118 & 71.7814\end{array}$

$107.7088 \quad 128.1495$

ZeroEnergy [ $\mathrm{kcal} / \mathrm{mol}]$

0.0

End

Fragment 16_r

Atom

End

GroundEnergy[kcal/mol] $\quad 10.537767033504355$

Mass[amu ] 1

$\begin{array}{cc}\text { Bimolecular } & 17 \\ \text { Fragment } & 17 \_m\end{array}$

RRHO

Geometry[angstrom]

C $\quad-1.755876$

21

C $\quad-1.558531$

$-2.804083$

$-2.496436$

0.927608

1.976577

3.350915

$-3.595210$

$-2.618823$

$-2.157555$

$-0.840446$

$-3.197849$

1.287570

0.853261

1.640692

2.032629

3.706362

3.315324

4.094173

$-0.405586$

$-0.409833$

Core

RigidRotor

SymmetryFactor $\quad 1.0$

End

Frequencies [1/cm ] 57

$3034.178 \quad 3043.04953064 .48413002 .4523 \quad 3023.3471$

3031.43452980 .64072994 .99322997 .05012946 .2755

$2947.234 \quad 2951.16821718 .13212929 .95012939 .4273$

1450.14691451 .54771461 .39531441 .05981442 .1009

1446.32941374 .67821422 .03441429 .14681347 .4947

1366.81471369 .05071254 .95791282 .23951327 .3147

1113.63171194 .85771231 .53671065 .48081071 .4461

$\begin{array}{lllll}1087.8142 & 982.2658 & 988.3382 & 1035.0759 & 864.1707\end{array}$

$\begin{array}{llllll}884.8527 & 931.738 & 714.4342 & 774.1376 & 837.491\end{array}$

$\begin{array}{llllll}403.0875 & 464.1563 & 527.5027 & 283.8602 & 308.5482\end{array}$

$\begin{array}{lllll}339.6874 & 124.1178 & 198.6981 & 229.4807 & 35.0551\end{array}$

84.196194 .2206

ZeroEnergy [ $\mathrm{kcal} / \mathrm{mol}]$

0.0

End

Fragment 17_r

Atom

Mass[amu ] 1

End

End

GroundEnergy [kcal/mol]

9.221879612009362

Bimolecular 18

Fragment 18_m 
RRHO

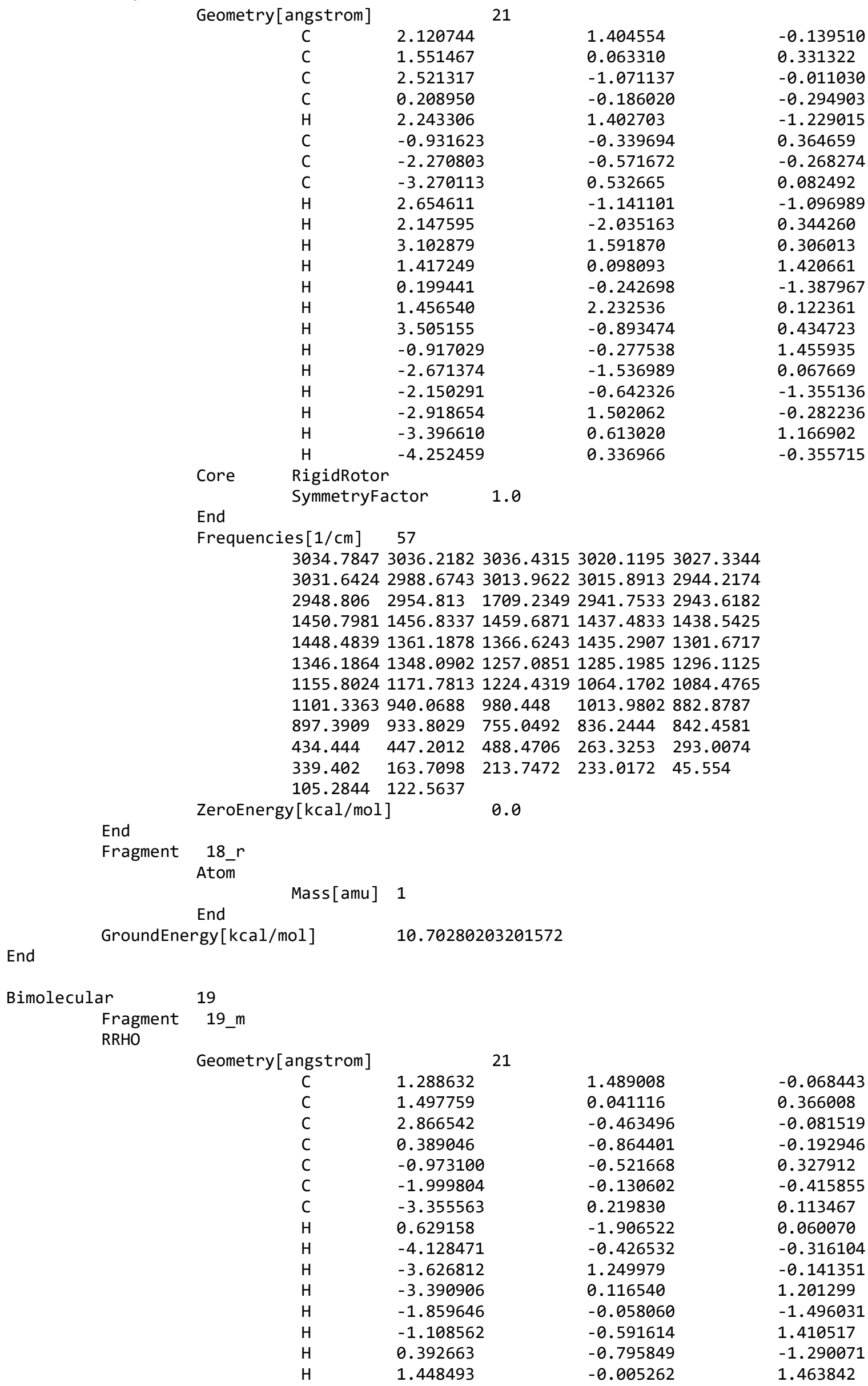




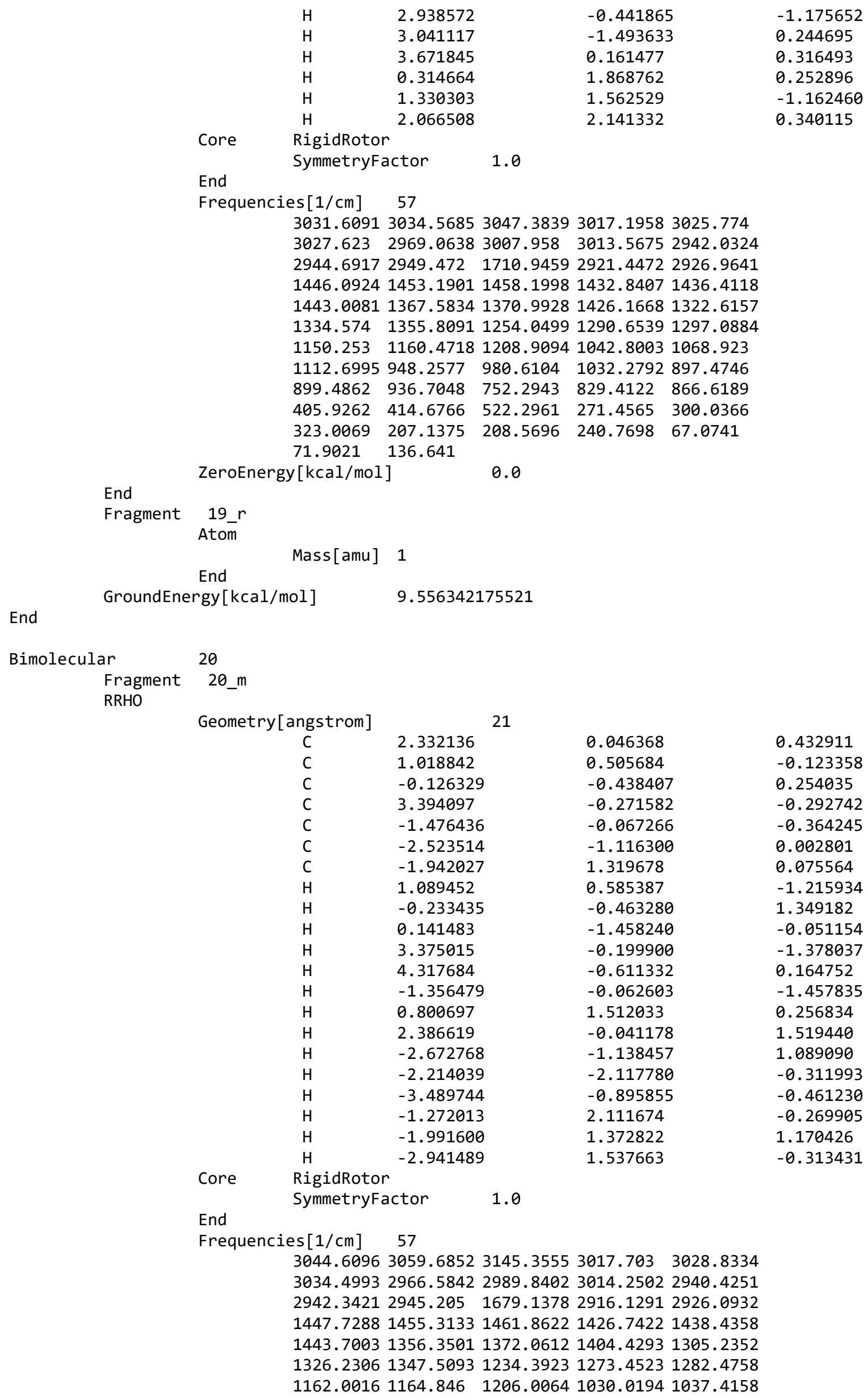


End

$\begin{array}{lll}1117.3691 & 940.3727 & 963.4587 \\ 924.2313 & 937.0689 & 740.4627 \\ 418.7868 & 452.3459 & 638.6896 \\ 388.7885 & 167.0917 & 224.9071 \\ 73.347 & 114.1725 & \\ y[\mathrm{kcal} / \mathrm{mol}] & 0.0\end{array}$

End

ZeroEnergy [ $\mathrm{kcal} / \mathrm{mol}]$

Fragment 20_r

Atom

End

GroundEnergy [kcal/mol]

Mass[amu ] 1

Bimolecular
Rragment $\quad 7$

Geometry [angstrom]

C

C

C

C

C

$\mathrm{H}$

$\mathrm{H}$

$\mathrm{H}$

$\mathrm{H}$

$\mathrm{H}$

$\mathrm{H}$

$\mathrm{H}$

$\mathrm{H}$

$\mathrm{H}$

$\mathrm{H}$

$\mathrm{H}$

Core

RigidRotor

End

SymmetryFactor

2.027445

0.768996

$-0.470667$

$-1.761414$

$-2.990109$

3.060028

2.064320

3.056574

3.943280

0.858888

0.633351

$-0.357146$

$-0.535108$

$-1.689406$

$-1.865512$

$-2.913465$

$-3.091356$

$-3.910096$

18

1008.9042898 .4081

$820.4723 \quad 894.1687$

$296.4298 \quad 340.6916$

$244.9754 \quad 54.0277$

0.0

Frequencies[1/cm] 48

$\begin{array}{lllll}3039.4159 & 3054.604 & 3140.7263 & 2990.706 & 3023.9214\end{array}$ 3033.07192953 .22712960 .38222975 .78542926 .1701 2932.39682941 .88391451 .77321459 .60621680 .4933 1429.71941436 .40281447 .49551353 .32111365 .3111 1404.42421278 .88751288 .14631309 .16231207 .0228 $1225.252 \quad 1268.28761050 .36211099 .11191162 .8666$ $1001.65871012 .30951022 .7635893 .0795 \quad 917.5899$ $\begin{array}{lllll}943.7369 & 705.2486 & 767.0259 & 883.0861 & 349.8544\end{array}$ $\begin{array}{lllll}442.6394 & 639.2018 & 169.0242 & 239.607 & 340.7328\end{array}$ $\begin{array}{lll}72.7499 & 90.7596 & 121.3154\end{array}$

ZeroEnergy [kcal/mol] 0.0

End

Fragment 7_r

RRHO
0.204874

$-0.589664$

0.298399

$-0.504401$

0.177163

0.861542

0.785520

$-1.234943$

$-1.254048$

0.965919

$-1.175240$

$-1.152334$

1.023221

1.046625

$-0.196871$
$-0.462644$
Geometry[angstrom]

$$
\text { C }
$$

$\mathrm{H}$

$\mathrm{H}$

$\mathrm{H}$

Core

RigidRotor

End SymmetryFactor

4

0.000000
0.000000
0.934781
-0.934781

6.0

Frequencies $[1 / \mathrm{cm}] \quad 6$ $3058.34563231 .11873231 .4024526 .3045 \quad 1368.0453$ 1368.0619

ZeroEnergy [kcal/mol]

End

nd


End

Bimolecular
Rragment
RRHO

Geometry[angstrom]

C

C

C

C

$\mathrm{H}$

$\mathrm{H}$

$\mathrm{H}$

$\mathrm{H}$

$\mathrm{H}$

$\mathrm{H}$

$\mathrm{H}$

$\mathrm{H}$

$\mathrm{H}$

$\mathrm{H}$

$\mathrm{H}$

Core

RigidRotor

End

SymmetryFactor

1.791061

3.167558

0.739170

$-0.643720$

$-1.670225$

$-3.079508$

3.194509

3.509690

1.657204

0.868318

3.893885

$-0.964555$

$-0.638290$

$-1.370230$

$-1.648027$

$-3.398752$

$-3.122049$

$-3.807717$

18

$-0.367796$

0.217551

0.097973

$-0.473730$

0.526993

$-0.052929$

1.088371

0.530440

$-1.244444$

0.977357

$-0.514259$

$-0.797200$

$-1.371263$

0.837102

1.433017

$-0.343601$

$-0.947794$

0.663903

1.0

Frequencies $[1 / \mathrm{cm}] \quad 48$

3029.70483034 .68273045 .93853009 .05333022 .5842

$\begin{array}{llllll}3028.236 & 2952.215 & 2967.8323 & 2989.7388 & 2925.8284\end{array}$

2946.09542949 .43871449 .26351459 .83091711 .6884

1432.76991444 .70721445 .91521365 .37911369 .321

1431.53721293 .81871300 .97491346 .55021212 .9046

1250.16651277 .61571071 .71971088 .45071148 .1536

$1011.717 \quad 1034.1921 \quad 1042.1997879 .2548 \quad 901.7974$

$\begin{array}{llllll}975.8123 & 719.0449 & 767.1406 & 848.9919 & 297.6656\end{array}$

$\begin{array}{lllll}379.4584 & 516.4705 & 203.3702 & 236.3147 & 278.7204\end{array}$

$\begin{array}{lll}73.5318 & 94.3721 & 136.6397\end{array}$

ZeroEnergy $[\mathrm{kcal} / \mathrm{mol}] \quad 0.0$

End

Fragment 8_r

RRHO

End

Geometry[angstrom]

C $\quad 0.000000$

4

$\begin{array}{lll}\mathrm{H} & 0.000000 & 1.079392 \\ \mathrm{H} & 0.934781 & -0.539696 \\ \mathrm{H} & -0.934781 & -0.539696\end{array}$

Core RigidRotor

SymmetryFactor

6.0

End

Frequencies [1/cm] 6

$3058.34563231 .11873231 .4024526 .3045 \quad 1368.0453$

1368.0619

ZeroEnergy $[\mathrm{kcal} / \mathrm{mol}]$

0.0

End

GroundEnergy [kcal/mol]

$-0.2610439519829835$

Bimolecular

9

Fragment 9_m

RRHO

Geometry [angstrom]

$\begin{array}{ll}C & -0.823453 \\ C & -0.928654 \\ C & -2.229580 \\ C & 0.274671\end{array}$

18

1.472318
0.000232
-0.603546
-0.794125

$-0.219884$

$-0.159105$

0.440894

0.369602

$-0.168536$

$-0.192102$

0.501340

$-1.151395$

$-0.856448$

1.076889

0.210518

1.370256

$-0.261994$

$-1.176454$

0.450157

0.814210

$-0.821720$

$-0.580577$
0.000062
-0.000124
-0.000124
-0.000124 


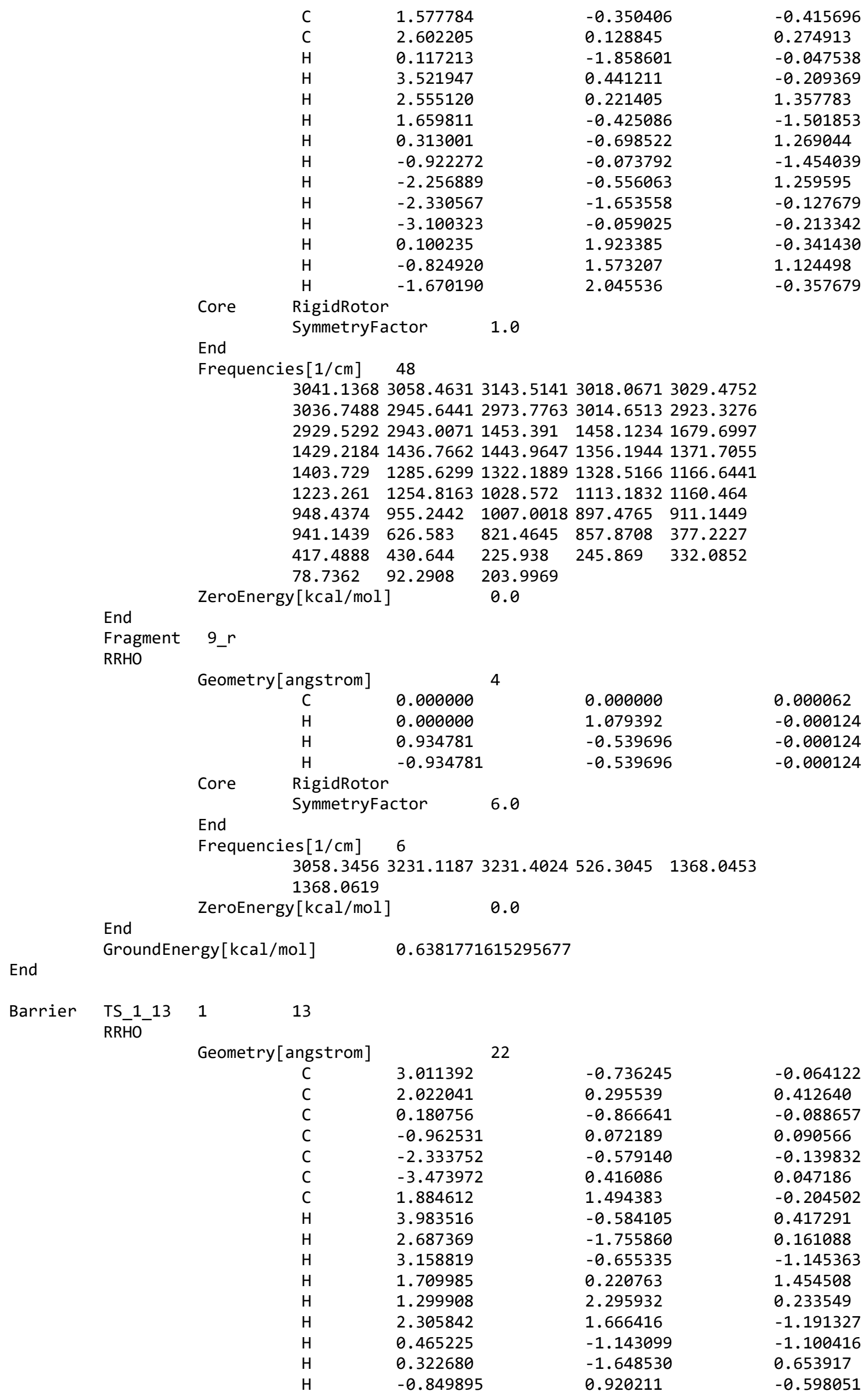




\begin{tabular}{|c|c|c|c|c|c|c|c|}
\hline & & $\mathrm{H}$ & -0.941431 & & 0.496126 & & 1.105369 \\
\hline & & $\mathrm{H}$ & -2.364613 & & -1.00212 & & -1.151158 \\
\hline & & H & -2.454037 & & -1.42321 & & 0.549969 \\
\hline & & $\mathrm{H}$ & -3.378280 & & 1.253196 & & -0.652016 \\
\hline & & $\mathrm{H}$ & -3.466517 & & 0.830990 & & 1.060484 \\
\hline & & $\mathrm{H}$ & -4.449856 & & -0.04839 & & -0.117514 \\
\hline & & RigidRoto & & & & & \\
\hline & & SymmetryF & actor & 0.5 & & & \\
\hline & & End & & & & & \\
\hline & & Frequencies $[1 / \mathrm{cm}]$ & 59 & & & & \\
\hline & & 3059.3245 & 3123.2275 & 3150.0456 & 3032.9531 & 3034.1098 & \\
\hline & & 3047.1033 & 3018.6368 & 3023.5309 & 3029.6985 & 2952.9595 & \\
\hline & & 2962.7938 & 2988.2556 & 2917.6557 & 2947.1145 & 2952.0536 & \\
\hline & & 1451.3436 & 1458.4464 & 1542.1316 & 1436.0915 & 1443.8032 & \\
\hline & & 1446.3221 & 1390.8138 & 1420.9397 & 1434.4886 & 1328.9358 & \\
\hline & & 1357.457 & 1364.2985 & 1248.1623 & 1257.6168 & 1284.7043 & \\
\hline & & 1146.4815 & 1168.3354 & 1242.3407 & 1022.2125 & 1062.4697 & \\
\hline & & 1085.1471 & 927.8195 & 975.293 & 1014.0825 & 868.822 & \\
\hline & & 895.8943 & 907.8554 & 777.5329 & 781.794 & 837.3792 & \\
\hline & & 540.8591 & 546.3868 & 707.3431 & 305.322 & 400.7278 & \\
\hline & & 410.9918 & 216.1383 & 235.9629 & 247.9283 & 91.2369 & \\
\hline & & 124.822 & 163.8785 & 46.7074 & 58.4824 & & \\
\hline & & ZeroEnergy [kcal/mol & & 9.6034053 & 388009186 & & \\
\hline & End & & & & & & \\
\hline Barrier & TS_1_16 & 16 & & & & & \\
\hline & RRHO & Geometry[angstrom] & & & & & \\
\hline & & $\begin{array}{c}\text { Geometry }[\text { angstrom }] \\
\text { C }\end{array}$ & 2.900225 & 22 & -0.77128 & & 0.037147 \\
\hline & & $\mathrm{C}$ & 1.682579 & & 0.119959 & & 0.026681 \\
\hline & & C & 0.369340 & & -0.60744 & & -0.167764 \\
\hline & & C & -0.883705 & & 0.238545 & & 0.003718 \\
\hline & & C & -2.161598 & & -0.59104 & & -0.071869 \\
\hline & & C & -3.415851 & & 0.260499 & & 0.086727 \\
\hline & & C & 1.802870 & & 1.454441 & & -0.123276 \\
\hline & & H & 3.809472 & & -0.20571 & & 0.252223 \\
\hline & & $\mathrm{H}$ & 2.799619 & & -1.56865 & & 0.779502 \\
\hline & & $\mathrm{H}$ & 3.014809 & & -1.24908 & & -0.943022 \\
\hline & & $\mathrm{H}$ & 1.473627 & & 0.129260 & & 1.849142 \\
\hline & & H & 2.777728 & & 1.930023 & & -0.080691 \\
\hline & & $\mathrm{H}$ & 0.938033 & & 2.100608 & & -0.226397 \\
\hline & & $\mathrm{H}$ & 0.381955 & & -1.05293 & & -1.174335 \\
\hline & & $\mathrm{H}$ & 0.335131 & & -1.45594 & & 0.529198 \\
\hline & & $\mathrm{H}$ & -0.918884 & & 1.020481 & & -0.766263 \\
\hline & & $\mathrm{H}$ & -0.836111 & & 0.757453 & & 0.971983 \\
\hline & & $\mathrm{H}$ & -2.191336 & & -1.12346 & & -1.031396 \\
\hline & & H & -2.134936 & & -1.36464 & & 0.706062 \\
\hline & & $\mathrm{H}$ & -3.471724 & & 1.022479 & & -0.697632 \\
\hline & & H & -3.414011 & & 0.779938 & & 1.050562 \\
\hline & & $\mathrm{H}$ & -4.326535 & & -0.34185 & & 0.032884 \\
\hline & & RigidRoto & & & & & \\
\hline & & SymmetryF & actor & 0.5 & & & \\
\hline & & End & & & & & \\
\hline & & Frequencies $[1 / \mathrm{cm}]$ & 59 & & & & \\
\hline & & 3049.0606 & 3071.5883 & 3160.0396 & 3015.9043 & 3023.1016 & \\
\hline & & 3034.0147 & 2952.9231 & 2966.2298 & 2986.3051 & 2941.1406 & \\
\hline & & 2950.3861 & 2952.7024 & 1601.3171 & 2917.1891 & 2927.6669 & \\
\hline & & 1447.607 & 1450.6626 & 1460.2107 & 1431.3785 & 1437.1292 & \\
\hline & & 1445.6028 & 1371.7444 & 1392.324 & 1423.1046 & 1334.6958 & \\
\hline & & 1362.1685 & 1365.9344 & 1258.5178 & 1270.9766 & 1286.2037 & \\
\hline & & 1105.4044 & 1202.525 & 1207.4201 & 1027.2025 & 1058.725 & \\
\hline & & 1100.1854 & 942.7646 & 981.6875 & 1016.8902 & 883.9475 & \\
\hline & & 889.005 & 909.4014 & 708.9657 & 765.174 & 833.8471 & \\
\hline & & 513.0244 & 550.3782 & 632.0377 & 427.9355 & 446.7109 & \\
\hline & & 479.2715 & 236.3191 & 290.8936 & 312.6137 & 122.1542 & \\
\hline & & 144.2305 & 173.3326 & 62.711 & 92.842 & & \\
\hline & & ZeroEnergy $[\mathrm{kcal} / \mathrm{mol}$ & & $15.50324 \mathrm{c}$ & 9707023482 & & \\
\hline
\end{tabular}


End

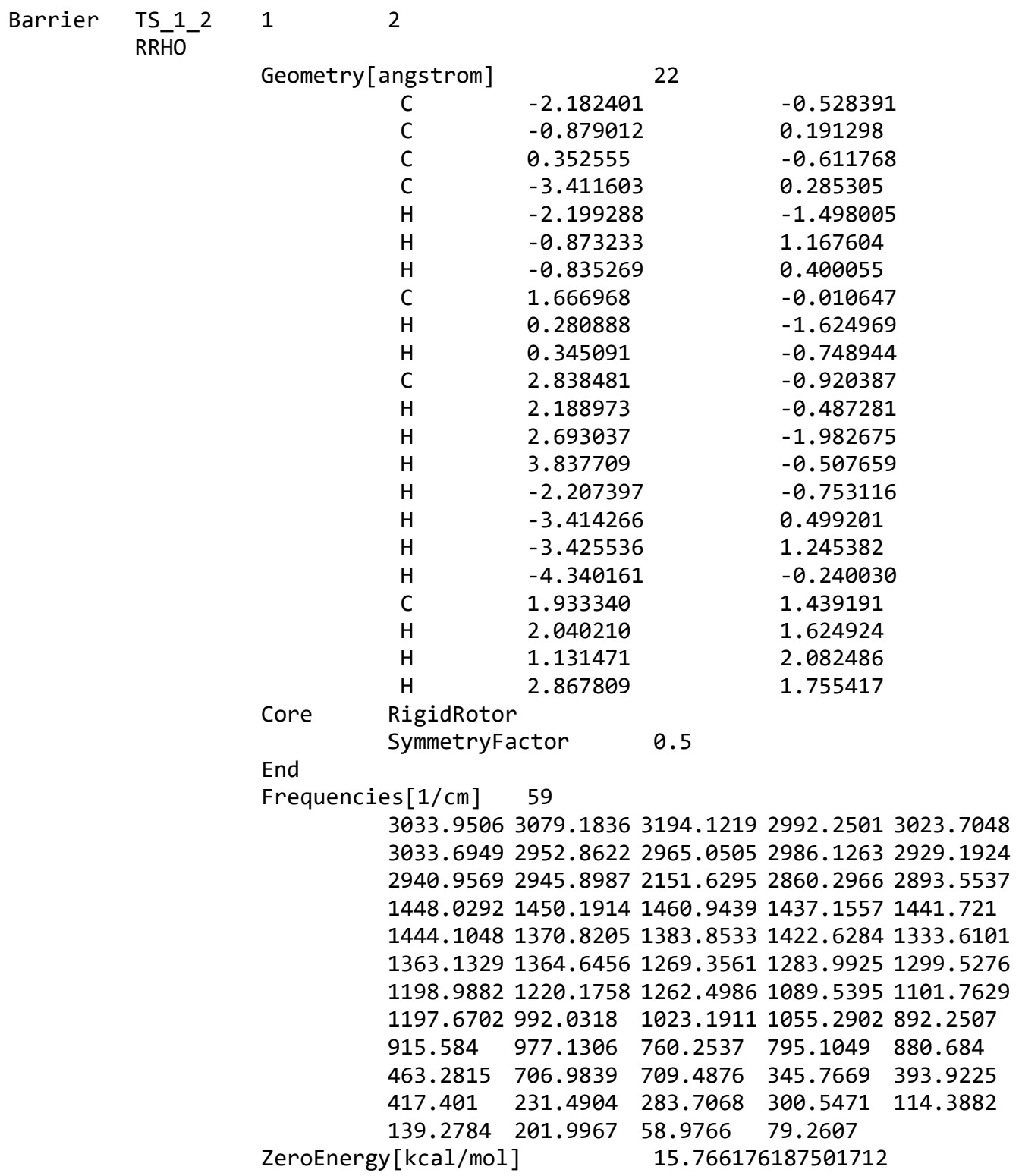

End

\begin{tabular}{|c|c|c|c|c|c|}
\hline \multirow[t]{20}{*}{ Barrier } & \multirow[t]{20}{*}{$\begin{array}{l}\mathrm{TS}_{\mathrm{R}}{ }^{1}{ }_{-} 3 \\
\mathrm{RHO}\end{array}$} & 1 & & & \\
\hline & & Geometry[angstrom] & & & \\
\hline & & $\mathrm{C}$ & -1.753205 & 1.418464 & 0.112888 \\
\hline & & C & -1.681122 & -0.008716 & -0.423822 \\
\hline & & C & -2.854205 & -0.872687 & 0.009085 \\
\hline & & C & -0.394411 & -0.287159 & 0.352892 \\
\hline & & $\mathrm{H}$ & -0.702926 & 0.946171 & 0.918412 \\
\hline & & C & 0.909501 & -0.142582 & -0.379011 \\
\hline & & C & 2.126721 & -0.117942 & 0.540365 \\
\hline & & C & 3.435514 & 0.003166 & -0.231220 \\
\hline & & $\mathrm{H}$ & -2.984950 & -0.810569 & 1.095961 \\
\hline & & $\mathrm{H}$ & -2.692386 & -1.923718 & -0.248615 \\
\hline & & $\mathrm{H}$ & -2.620047 & 1.694734 & 0.711338 \\
\hline & & $\mathrm{H}$ & -1.540541 & -0.064183 & -1.509363 \\
\hline & & $\mathrm{H}$ & -0.446925 & -1.074188 & 1.110498 \\
\hline & & $\mathrm{H}$ & -1.334864 & 2.211356 & -0.502501 \\
\hline & & $\mathrm{H}$ & -3.786815 & -0.543155 & -0.458337 \\
\hline & & $\mathrm{H}$ & 0.883550 & 0.780076 & -0.979103 \\
\hline & & $\mathrm{H}$ & 1.018710 & -0.963457 & -1.107387 \\
\hline & & $\mathrm{H}$ & 2.134103 & -1.030804 & 1.149475 \\
\hline
\end{tabular}




\begin{tabular}{|c|c|c|c|c|c|c|c|}
\hline & & $\mathrm{H}$ & 2.023558 & & 0.717491 & & 1.243539 \\
\hline & & $\mathrm{H}$ & 3.452263 & & 0.920400 & & -0.829139 \\
\hline & & $\mathrm{H}$ & 3.563835 & & -0.839595 & & -0.918469 \\
\hline & & $\mathrm{H}$ & 4.300674 & & 0.024178 & & 0.436623 \\
\hline & & RigidRoto & & & & & \\
\hline & & SymmetryF & actor & 0.25 & & & \\
\hline & & End & & & & & \\
\hline & & Frequencies $[1 / \mathrm{cm}]$ & 59 & & & & \\
\hline & & 3032.6198 & 3032.9199 & 3126.2945 & 3019.2793 & 3019.6278 & \\
\hline & & 3023.373 & 2968.0039 & 2983.4713 & 3004.8676 & 2945.23 & \\
\hline & & 2946.1108 & 2952.6021 & 1752.6927 & 2891.6153 & 2930.8184 & \\
\hline & & 1449.0722 & 1450.6081 & 1458.1555 & 1426.693 & 1443.2325 & \\
\hline & & 1446.647 & 1363.9219 & 1365.5186 & 1384.6533 & 1296.5028 & \\
\hline & & 1330.5577 & 1357.4884 & 1231.6497 & 1275.242 & 1283.6735 & \\
\hline & & 1156.5799 & 1206.2027 & 1215.9523 & 1071.6209 & 1101.4083 & \\
\hline & & 1119.8797 & 1023.561 & 1037.9882 & 1055.9277 & 894.1198 & \\
\hline & & 907.9488 & 917.3187 & 840.1821 & 875.3562 & 882.4287 & \\
\hline & & 628.1699 & 708.0747 & 765.9457 & 350.9296 & 413.0711 & \\
\hline & & 453.9248 & 247.5093 & 274.8057 & 308.438 & 113.3876 & \\
\hline & & 143.1899 & 236.6943 & 60.5908 & 89.5623 & & \\
\hline & & ZeroEnergy $[\mathrm{kcal} / \mathrm{mol}$ & & 18.78951 & 6958501846 & & \\
\hline & End & & & & & & \\
\hline Barrier & TS_1_4 & 1 & & & & & \\
\hline & & Geometry[angstrom] & & 22 & & & \\
\hline & & $\mathrm{C}$ & -0.91846 & & 1.379473 & & 0.316844 \\
\hline & & C & -1.35955 & & 0.090803 & & -0.354066 \\
\hline & & C & -0.565537 & & -1.007736 & & 0.388740 \\
\hline & & C & 0.800032 & & -0.405103 & & 0.662253 \\
\hline & & C & 1.786075 & & -0.415511 & & -0.479016 \\
\hline & & C & 3.045758 & & 0.385802 & & -0.168491 \\
\hline & & C & -2.86506 & & -0.144734 & & -0.329315 \\
\hline & & $\mathrm{H}$ & 0.313300 & & 0.855855 & & 0.703524 \\
\hline & & $\mathrm{H}$ & -1.02297 & & 0.111529 & & -1.399083 \\
\hline & & $\mathrm{H}$ & -1.39670 & & 1.583581 & & 1.277295 \\
\hline & & $\mathrm{H}$ & -0.79126 & & 2.270840 & & -0.294010 \\
\hline & & $\mathrm{H}$ & -3.40580 & & 0.632209 & & -0.877304 \\
\hline & & $\mathrm{H}$ & -3.230216 & & -0.14307 & & 0.704161 \\
\hline & & $\mathrm{H}$ & -3.12066 & & -1.11283 & & -0.773690 \\
\hline & & $\mathrm{H}$ & -1.07612 & & -1.23216 & & 1.334241 \\
\hline & & $\mathrm{H}$ & -0.51737 & & -1.94248 & & -0.184607 \\
\hline & & $\mathrm{H}$ & 1.248676 & & $-0.63142 \varepsilon$ & & 1.631757 \\
\hline & & $\mathrm{H}$ & 2.053393 & & $-1.45621 S$ & & -0.718922 \\
\hline & & $\mathrm{H}$ & 1.303130 & & -0.01639 & & -1.381350 \\
\hline & & $\mathrm{H}$ & 3.552487 & & -0.01579 & & 0.715043 \\
\hline & & $\mathrm{H}$ & 2.795416 & & 1.430639 & & 0.041152 \\
\hline & & $\mathrm{H}$ & 3.755180 & & 0.367737 & & -0.999901 \\
\hline & & RigidRoto & & & & & \\
\hline & & SymmetryF & actor & 0.25 & & & \\
\hline & & End & & & & & \\
\hline & & Frequencies $[1 / \mathrm{cm}]$ & 59 & & & & \\
\hline & & 3025.4529 & 3035.3011 & 3097.9308 & 3015.4262 & 3021.8472 & \\
\hline & & 3024.9427 & 2957.6219 & 2984.2921 & 3002.7907 & 2941.4063 & \\
\hline & & 2944.7413 & 2953.2411 & 1603.7917 & 2909.7439 & 2937.4507 & \\
\hline & & 1448.3926 & 1448.9552 & 1454.912 & 1431.9185 & 1435.7141 & \\
\hline & & 1445.9991 & 1364.2751 & 1366.3423 & 1407.8138 & 1318.1595 & \\
\hline & & 1328.1181 & 1360.7135 & 1256.9404 & 1269.1856 & 1302.6313 & \\
\hline & & 1148.451 & 1206.037 & 1235.4551 & 1087.9779 & 1103.6223 & \\
\hline & & 1138.3394 & 1002.4248 & 1016.6266 & 1037.8212 & 910.306 & \\
\hline & & 923.8782 & 966.5516 & 820.7028 & 856.0731 & 891.7528 & \\
\hline & & 598.1653 & 738.9633 & 795.3936 & 402.8799 & 476.4717 & \\
\hline & & 565.7429 & 247.0948 & 283.2174 & 366.0935 & 123.0228 & \\
\hline & & 229.5677 & 237.1321 & 71.7937 & 97.5249 & & \\
\hline & & ZeroEnergy $[\mathrm{kcal} / \mathrm{mol}$ & & 2.897211 & 3614975505 & & \\
\hline
\end{tabular}




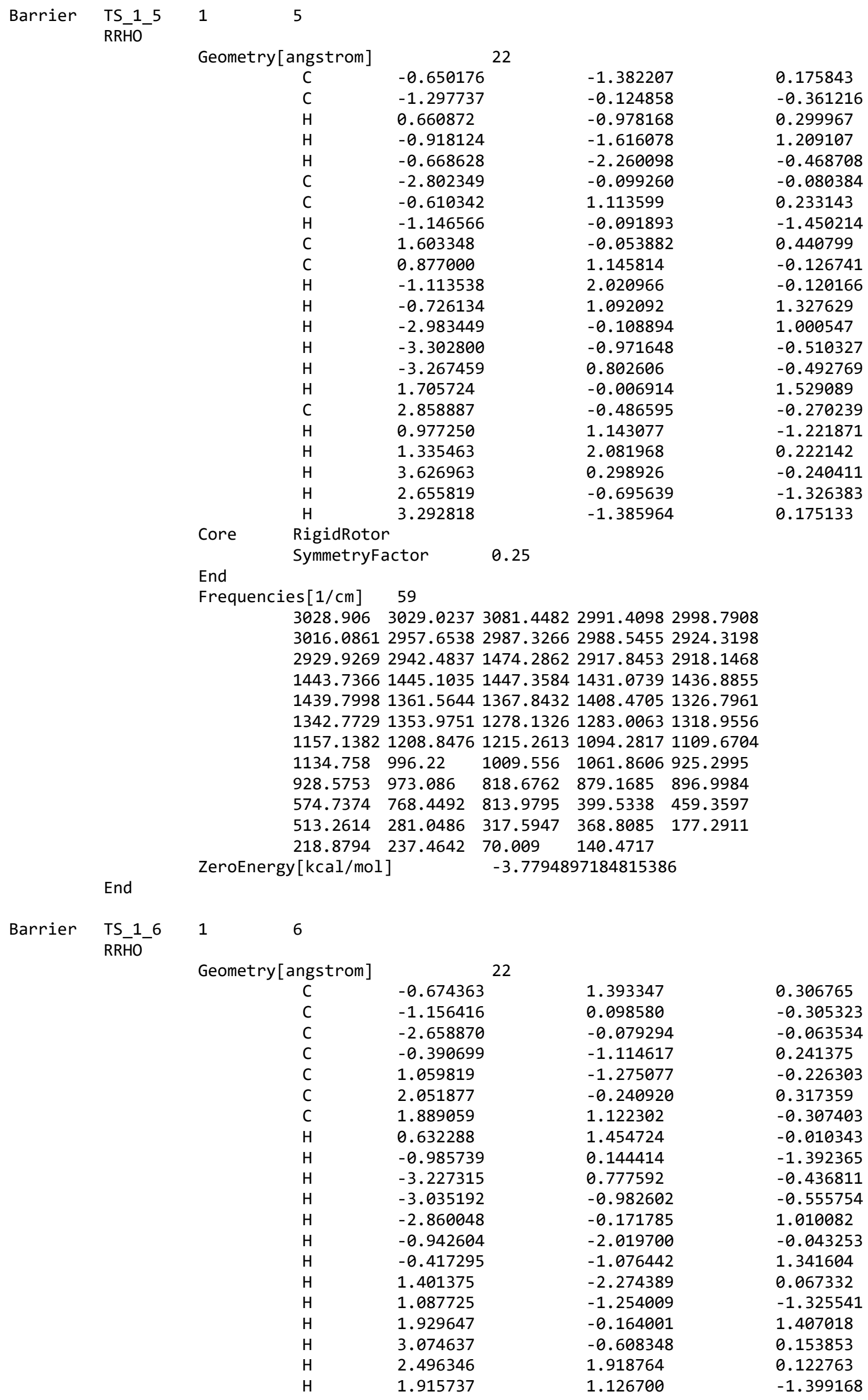




\begin{tabular}{|c|c|c|c|c|c|c|c|}
\hline & & $\mathrm{H}$ & -0.685421 & & 1.402142 & & 1.400101 \\
\hline & & $\mathrm{H}$ & -1.106585 & & 2.301013 & & -0.117139 \\
\hline & & RigidRoto & & & & & \\
\hline & & SymmetryF & actor & 0.5 & & & \\
\hline & & End & & & & & \\
\hline & & Frequencies $[1 / \mathrm{cm}]$ & 59 & & & & \\
\hline & & 3028.2736 & 3068.8049 & 3079.2459 & 2984.2075 & 2996.5115 & \\
\hline & & 3015.8065 & 2958.3278 & 2969.1721 & 2983.2641 & 2921.8007 & \\
\hline & & 2928.6793 & 2942.5355 & 1449.5313 & 2908.5797 & 2919.5489 & \\
\hline & & 1436.905 & 1441.6063 & 1445.2784 & 1405.987 & 1406.2295 & \\
\hline & & 1430.97 & 1356.4104 & 1361.4466 & 1390.9468 & 1328.8205 & \\
\hline & & 1341.4985 & 1343.9814 & 1254.536 & 1282.2723 & 1326.2596 & \\
\hline & & 1158.8229 & 1205.5973 & 1226.4985 & 1080.5829 & 1129.0238 & \\
\hline & & 1140.0406 & 982.6994 & 1010.7092 & 1056.7928 & 919.2656 & \\
\hline & & 935.053 & 973.2528 & 823.936 & 874.0447 & 906.5198 & \\
\hline & & 591.8952 & 750.9402 & 791.1583 & 434.264 & 499.4998 & \\
\hline & & 560.6933 & 312.2887 & 352.734 & 398.0278 & 226.0988 & \\
\hline & & 256.3682 & 287.9882 & 83.1911 & 120.7361 & & \\
\hline & & ZeroEnergy $[\mathrm{kcal} / \mathrm{mol}$ & & $-2.17996 \varepsilon$ & 8002989257 & & \\
\hline & End & & & & & & \\
\hline Barrier & TS_1_7 & 1 & & & & & \\
\hline & RRHO & & & & & & \\
\hline & & Geometry[angstrom] & & 22 & & & \\
\hline & & $\mathrm{C}$ & 3.054425 & & -1.200467 & & 0.036079 \\
\hline & & C & 1.518110 & & 0.413245 & & 0.366554 \\
\hline & & $\mathrm{C}$ & 0.345793 & & -0.344714 & & -0.205813 \\
\hline & & C & -0.990776 & & 0.257547 & & 0.234307 \\
\hline & & $\mathrm{C}$ & -2.190488 & & $-0.51762 \varepsilon$ & & -0.299517 \\
\hline & & C & -3.517681 & & 0.093195 & & 0.135897 \\
\hline & & C & 2.017242 & & 1.514704 & & -0.242751 \\
\hline & & $\mathrm{H}$ & 3.981999 & & -0.695767 & & 0.272194 \\
\hline & & $\mathrm{H}$ & 2.692243 & & -1.930684 & & 0.750667 \\
\hline & & $\mathrm{H}$ & 2.850253 & & -1.391277 & & -1.010251 \\
\hline & & $\mathrm{H}$ & 1.690662 & & 0.293349 & & 1.436256 \\
\hline & & $\mathrm{H}$ & 2.735109 & & 2.163548 & & 0.246452 \\
\hline & & $\mathrm{H}$ & 1.758159 & & 1.745527 & & -1.272970 \\
\hline & & $\mathrm{H}$ & 0.408437 & & -0.329836 & & -1.302267 \\
\hline & & $\mathrm{H}$ & 0.380315 & & -1.398305 & & 0.096729 \\
\hline & & $\mathrm{H}$ & -1.041091 & & 1.301721 & & -0.101033 \\
\hline & & $\mathrm{H}$ & -1.033516 & & 0.288633 & & 1.332060 \\
\hline & & $\mathrm{H}$ & -2.137605 & & -0.552887 & & -1.395379 \\
\hline & & $\mathrm{H}$ & -2.128969 & & -1.558991 & & 0.042079 \\
\hline & & $\mathrm{H}$ & -3.606576 & & 1.125029 & & -0.219234 \\
\hline & & $\mathrm{H}$ & -3.597581 & & 0.113538 & & 1.227729 \\
\hline & & $\mathrm{H}$ & -4.371594 & & -0.468892 & & -0.251566 \\
\hline & & RigidRoto & & & & & \\
\hline & & SymmetryF & actor & 0.5 & & & \\
\hline & & End & & & & & \\
\hline & & Frequencies $[1 / \mathrm{cm}]$ & 59 & & & & \\
\hline & & 3149.0644 & 3178.802 & 3189.5936 & 3033.7845 & 3047.4809 & \\
\hline & & 3056.5679 & 2995.2724 & 3019.8712 & 3024.6455 & 2953.1681 & \\
\hline & & 2962.2168 & 2979.1746 & 2928.8461 & 2938.3711 & 2944.6121 & \\
\hline & & 1450.762 & 1461.9189 & 1539.1176 & 1433.7232 & 1439.3468 & \\
\hline & & 1450.4975 & 1372.1673 & 1378.6363 & 1390.3248 & 1309.0112 & \\
\hline & & 1351.7692 & 1366.3893 & 1242.8859 & 1273.4857 & 1283.8897 & \\
\hline & & 1156.896 & 1207.4189 & 1226.4531 & 1017.3461 & 1050.1851 & \\
\hline & & 1096.6441 & 916.8528 & 973.2793 & 999.5575 & 855.9028 & \\
\hline & & 882.0461 & 890.1401 & 708.1453 & 766.4173 & 810.585 & \\
\hline & & 496.455 & 525.768 & 560.1723 & 347.4222 & 388.4484 & \\
\hline & & 422.2689 & 185.1506 & 234.9272 & 248.9802 & 103.6609 & \\
\hline & & 124.6661 & 133.0985 & 49.1063 & 82.7068 & & \\
\hline & & ZeroEnergy $[\mathrm{kcal} / \mathrm{mol}$ & & 11.78902 & 0976519854 & & \\
\hline & End & & & & & & \\
\hline Barrier & $\begin{array}{l}\text { TS_2_11 } \\
\text { RRHO }\end{array}$ & 11 & & & & & \\
\hline
\end{tabular}




Geometry [an

gstrom ]

4.456713

C $\quad 3.629305$

C $\quad 2.271684$

$\mathrm{H} \quad 3.731674$

$\begin{array}{ll}\mathrm{H} & 3.731725\end{array}$

C $\quad 1.114908$

H 2.215020

$\mathrm{H} \quad 2.213347$

C $\quad-0.636871$

H $\quad 0.903944$

$\mathrm{H} \quad-0.249973$

H $\quad 0.890797$

C $\quad-1.802531$

C $\quad-2.450805$

C $\quad-2.440882$

H $\quad-0.256963$

$\mathrm{H} \quad-2.447522$

$\mathrm{H} \quad-1.941009$

$\begin{array}{ll}H & -3.502787\end{array}$

$\mathrm{H} \quad-1.923862$

$\mathrm{H} \quad-2.437590$

$\mathrm{H} \quad-3.492356$

Core RigidRotor

End

SymmetryFactor

Frequencies $[1 / \mathrm{cm}] \quad 59$

3043.69883127 .64573135 .14383036 .24443037 .7356 3042.56912979 .34793027 .58493033 .20682951 .8892 $\begin{array}{lllll}2972.2631 & 2978.3771 & 2924.9912 & 2927.798 & 2933.0664\end{array}$ 1447.50031455 .25361565 .80951433 .56111434 .8768 1447.36821417 .55911422 .60331433 .18241354 .5463 $1365.2297 \quad 1375.438 \quad 1277.05551295 .3323 \quad 1354.0943$ $1082.927 \quad 1170.16441271 .67281023 .47861036 .8684$ $\begin{array}{llllll}1056.389 & 932.8632 & 958.8123 & 997.7121 & 876.6716\end{array}$ $\begin{array}{llllll}882.2559 & 917.8176 & 736.4026 & 803.1495 & 804.8235\end{array}$ $\begin{array}{llllll}424.5831 & 517.1245 & 718.8171 & 337.1745 & 362.8441\end{array}$ $\begin{array}{lllll}416.3811 & 141.7881 & 174.3601 & 246.3302 & 78.3651\end{array}$ $\begin{array}{llll}107.0505 & 139.4707 & 35.698 & 48.7152\end{array}$

End ZeroEnergy $[\mathrm{kcal} / \mathrm{mol}]$

5.046431399028768
Barrier TS_2_16 2

RRHO
Geometry [angstrom]

$\mathrm{H}$

C

4. 282283

2. 160837

3.298287

3.230534

0.798309

2.203957

2.267030

$-0.359072$

0.673897

$-0.268000$

0.757649

$-1.704207$

$-2.490742$

$-2.072513$

$-0.268929$

$-1.850202$

$-2.236478$

$-3.472371$

$-1.969650$

$-1.405923$

$-3.097741$
22

$\begin{array}{ll}0.102997 & -0.054757 \\ -0.159232 & -0.480197 \\ 0.262758 & 0.428192 \\ -1.241339 & -0.646890 \\ 0.325341 & -1.458730 \\ -0.093953 & -0.155899 \\ 1.344756 & 0.606612 \\ -0.213461 & 1.411585 \\ 0.324697 & 0.759198 \\ 0.392211 & -1.133422 \\ -0.214484 & 1.713470 \\ -1.175442 & -0.339995 \\ 0.050926 & 0.148393 \\ 1.049703 & -0.281554 \\ -1.387115 & -0.059080 \\ 1.393719 & 0.985465 \\ 1.363228 & -2.111790 \\ 2.087242 & -0.084282 \\ 0.852668 & -0.702876 \\ -1.954486 & 0.873282 \\ -1.858745 & -0.791463 \\ -1.490909 & -0.420533\end{array}$

0.415903

$-0.300421$

0.416002

$-0.960474$

$-0.915053$

$-0.528722$

1.095052

1.048800

0.896864

$-1.053784$

1.320572

$-1.088698$

0.207501

$-0.248677$

$-0.257126$

1. 326859

$-1.345274$

0.150194

0.060167

0.135493

$-1.354183$

0.051899 


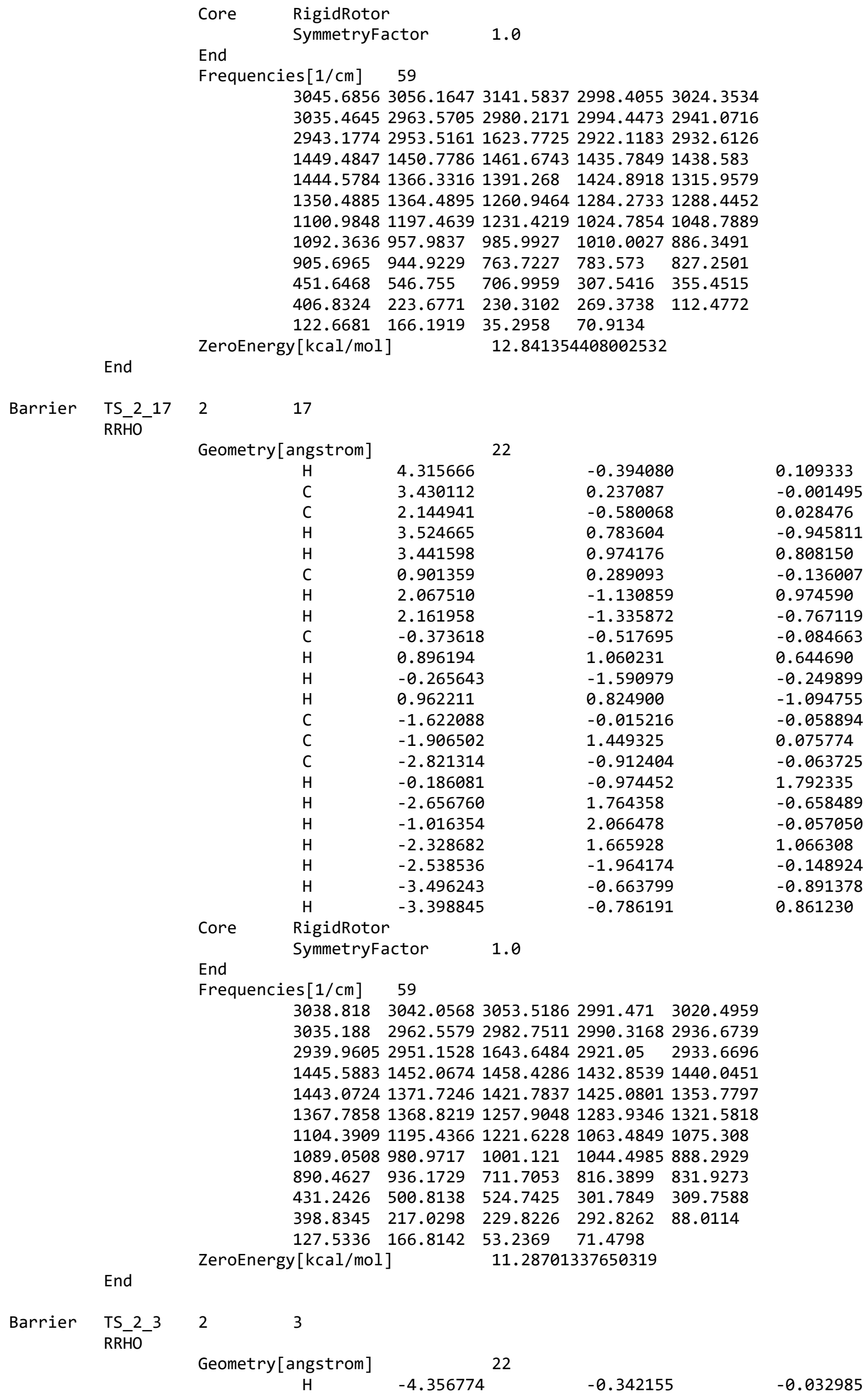




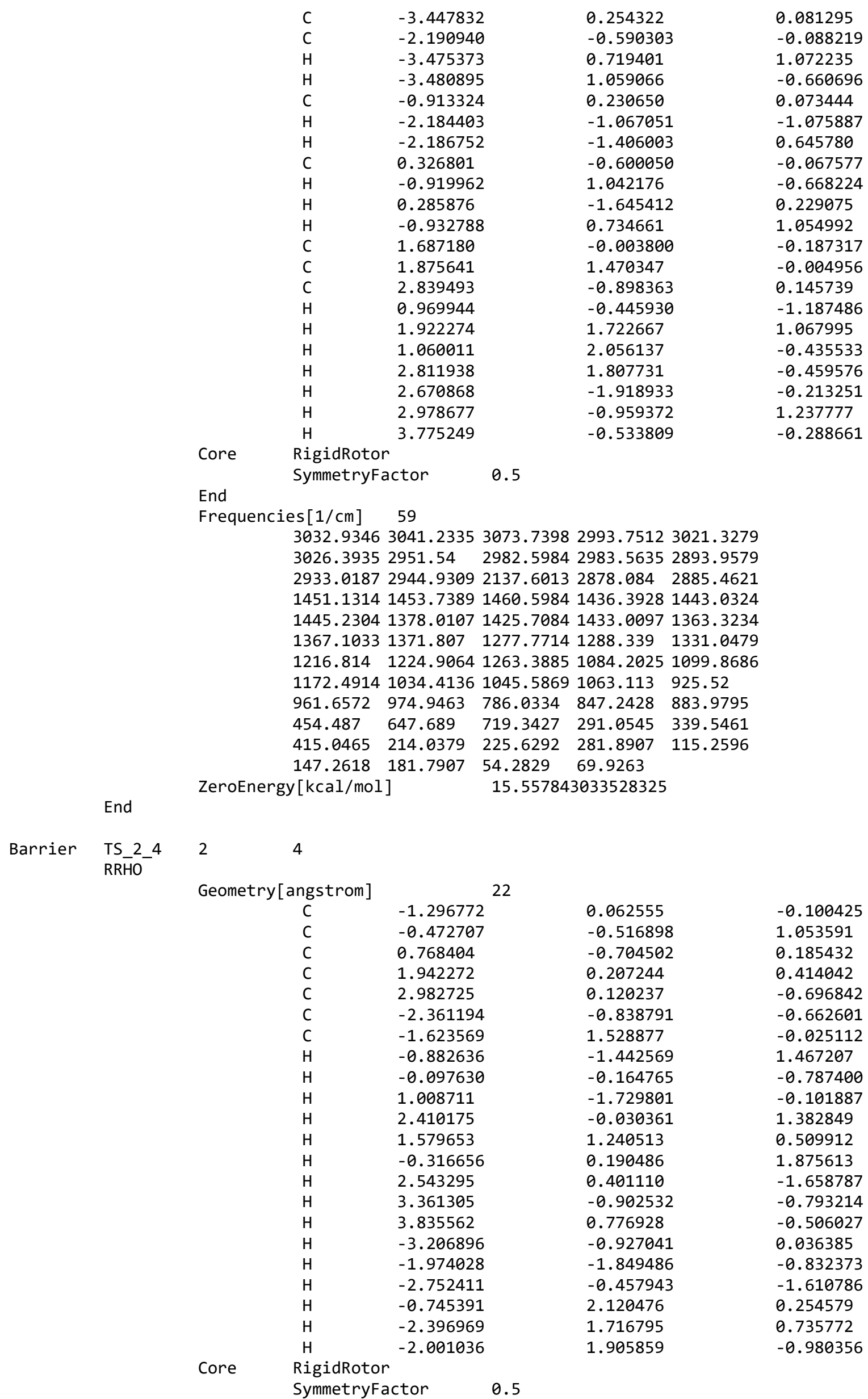


End

End

Frequencies $[1 / \mathrm{cm}] \quad 59$

3026.78063031 .60733035 .20083016 .51573023 .9159

$3026.41712964 .41632984 .41 \quad 2987.7662 \quad 2910.4014$ 2944.97272954 .82421737 .50712900 .88152907 .1583

$1446.28061446 .8968 \quad 1454.736 \quad 1434.03891434 .8435$

1444.23371365 .39781426 .09041429 .71311345 .1124

1358.52481364 .02871249 .08151264 .70161315 .6608

$1198.68151222 .55261243 .844 \quad 1072.54431088 .6911$

$1151.2708990 .8528 \quad 1015.62171055 .5042922 .0164$

$\begin{array}{lllll}951.3124 & 960.5814 & 788.2682 & 847.6509 & 898.8298\end{array}$

$\begin{array}{lllll}565.222 & 705.6029 & 773.0816 & 336.3399 & 352.8381\end{array}$

$\begin{array}{lllll}423.745 & 216.7427 & 235.0533 & 294.5018 & 110.8613\end{array}$

$\begin{array}{llll}202.2522 & 206.5136 & 59.3623 & 80.8474\end{array}$

ZeroEnergy [kcal/mol]

15.628751607027647

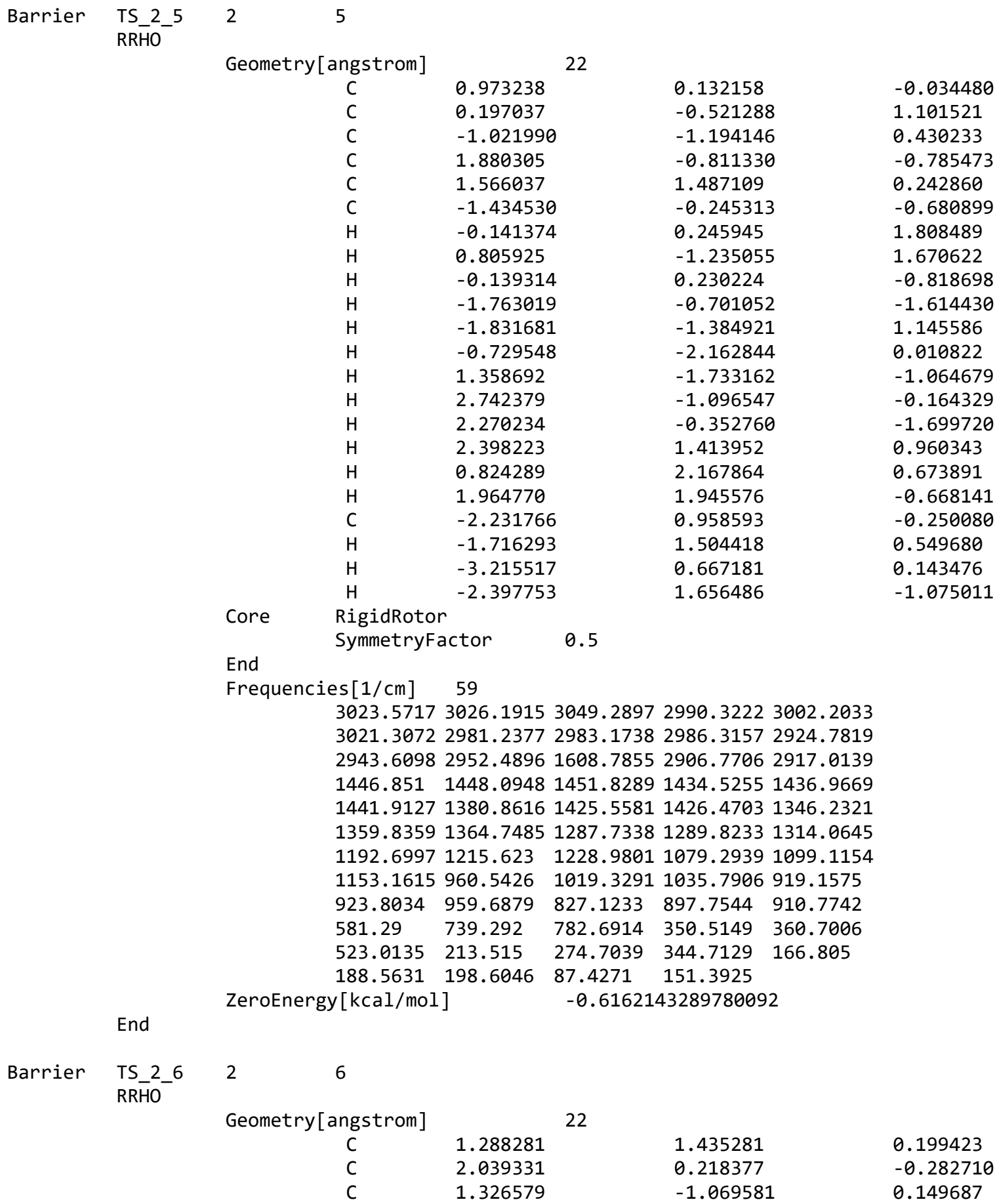




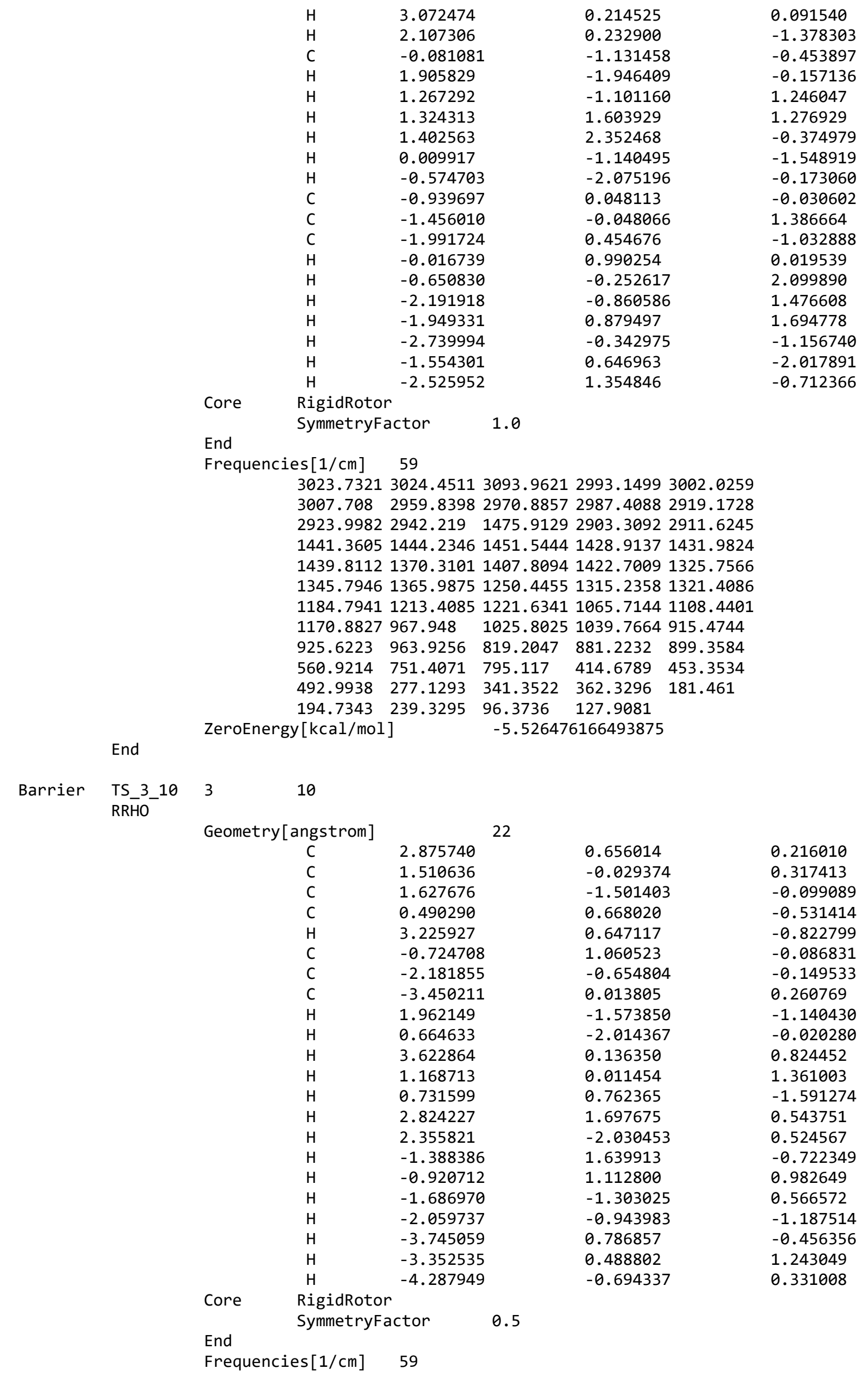


$3053.98923126 .39853147 .17193036 .259 \quad 3037.0626$ 3043.35543016 .39663019 .55433030 .25012946 .7359 $2981.937 \quad 3015.53352920 .861 \quad 2936.0105 \quad 2943.0269$ 1449.38541458 .53521548 .80081436 .99871437 .3791 $1441.07451394 .29451424 .90141435 .211 \quad 1346.3505$ 1358.78651364 .16511244 .17311278 .17571294 .7863 1151.89821175 .78731183 .45441005 .69461047 .9143 $\begin{array}{llllll}1094.4935 & 935.7468 & 941.1709 & 995.9044 & 882.2636\end{array}$ $\begin{array}{lllll}891.0009 & 914.9679 & 750.0771 & 789.2601 & 799.9855\end{array}$ $\begin{array}{llllll}487.3765 & 535.8711 & 723.4148 & 311.7361 & 335.6736\end{array}$ $\begin{array}{lllll}379.8952 & 203.9521 & 207.0116 & 234.8888 & 72.9609\end{array}$ $\begin{array}{llll}150.1968 & 169.3672 & 20.2433 & 57.0063\end{array}$

End ZeroEnergy [ $\mathrm{kcal} / \mathrm{mol}]$

7.448537765027897

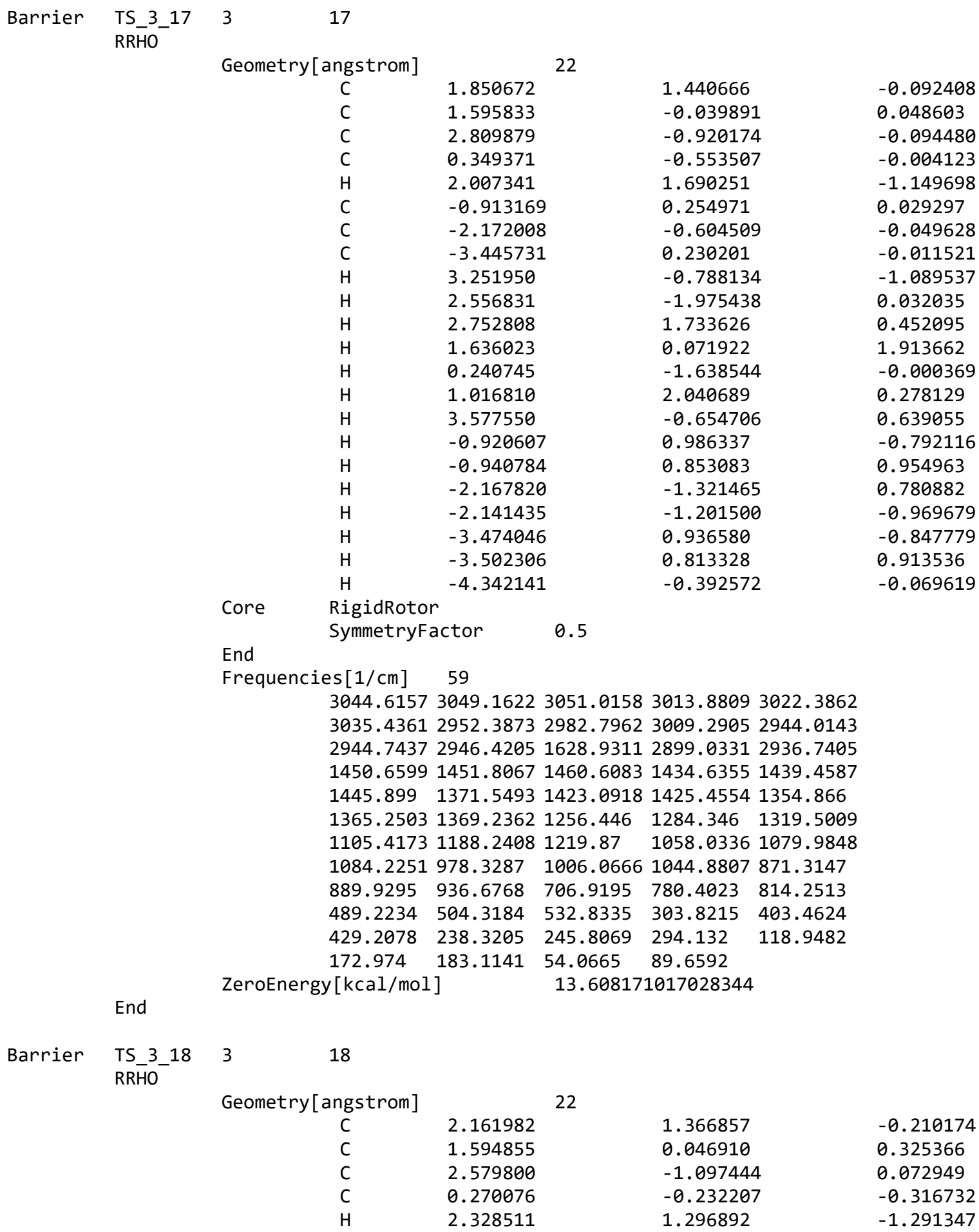




\begin{tabular}{|c|c|c|c|c|c|c|}
\hline & & $\mathrm{C}$ & -0.911159 & & -0.200803 & 0.323283 \\
\hline & & C & -2.229514 & & -0.553293 & -0.311589 \\
\hline & & C & -3.384315 & & 0.354936 & 0.105643 \\
\hline & & $\mathrm{H}$ & 2.738644 & & -1.229825 & -1.003558 \\
\hline & & $\mathrm{H}$ & 2.207385 & & -2.042691 & 0.476883 \\
\hline & & $\mathrm{H}$ & 3.121227 & & 1.600976 & 0.262117 \\
\hline & & $\mathrm{H}$ & 1.433800 & & 0.148024 & 1.407025 \\
\hline & & $\mathrm{H}$ & 0.281190 & & -0.389023 & -1.398445 \\
\hline & & $\mathrm{H}$ & 1.470998 & & 2.194736 & -0.026947 \\
\hline & & $\mathrm{H}$ & 3.551280 & & -0.883609 & 0.529499 \\
\hline & & $\mathrm{H}$ & -1.095414 & & 1.695374 & 0.474369 \\
\hline & & $\mathrm{H}$ & -0.905696 & & -0.128895 & 1.413496 \\
\hline & & $\mathrm{H}$ & -2.472313 & & -1.585862 & -0.026586 \\
\hline & & $\mathrm{H}$ & -2.116426 & & -0.552683 & -1.401421 \\
\hline & & $\mathrm{H}$ & -3.239486 & & 1.371604 & -0.270001 \\
\hline & & $\mathrm{H}$ & -3.455660 & & 0.415383 & 1.196649 \\
\hline & & $\mathrm{H}$ & -4.338385 & & -0.020141 & -0.274204 \\
\hline & & RigidRoto & & & & \\
\hline & & SymmetryF & actor & 0.5 & & \\
\hline & & End & & & & \\
\hline & & Frequencies $[1 / \mathrm{cm}]$ & 59 & & & \\
\hline & & 3033.11 & 3035.8304 & 3038.0099 & 3024.47483029 .5581 & \\
\hline & & 3031.647 & 2987.7418 & 3017.7386 & 3021.36142944 .9362 & \\
\hline & & 2948.6465 & 2955.9495 & 1627.1841 & 2937.57492942 .9936 & \\
\hline & & 1451.0187 & 1453.6571 & 1458.7185 & $1436.775 \quad 1438.7938$ & \\
\hline & & 1446.2623 & 1364.2405 & 1366.7002 & 1430.03751300 .4165 & \\
\hline & & 1347.4925 & 1350.3953 & 1251.9124 & 1272.28061293 .7694 & \\
\hline & & 1154.7939 & 1172.1839 & 1221.485 & 1066.17781082 .2949 & \\
\hline & & 1096.0009 & 942.1625 & 993.1535 & $1023.038 \quad 884.7589$ & \\
\hline & & 899.5069 & 933.2246 & 746.4456 & $835.3881 \quad 848.5454$ & \\
\hline & & 444.5534 & 479.1747 & 521.2407 & $323.0064 \quad 416.1079$ & \\
\hline & & 427.5696 & 239.3625 & 262.1095 & $285.2342 \quad 110.4697$ & \\
\hline & & 156.7321 & 218.1563 & 47.6102 & 99.5253 & \\
\hline & & ZeroEnergy $[\mathrm{kcal} / \mathrm{mol}$ & & 14.31600 & 1733024732 & \\
\hline & End & & & & & \\
\hline Barrier & TS_3_4 & 3 & & & & \\
\hline & $\mathrm{RR} \overline{\mathrm{H}} \mathrm{O}$ & & & & & \\
\hline & & Geometry[angstrom] & & 22 & & \\
\hline & & $\mathrm{C}$ & -1.898149 & & 1.425799 & 0.145041 \\
\hline & & $C$ & -1.625216 & & -0.002526 & -0.346966 \\
\hline & & C & -2.795892 & & -0.922380 & -0.009706 \\
\hline & & C & -0.335851 & & -0.514392 & 0.227181 \\
\hline & & C & 0.961229 & & -0.027900 & -0.303293 \\
\hline & & C & 2.194280 & & -0.045654 & 0.543492 \\
\hline & & C & 3.482329 & & 0.019355 & -0.271470 \\
\hline & & $\mathrm{H}$ & 0.435442 & & -1.185050 & -0.577257 \\
\hline & & $\mathrm{H}$ & 3.563750 & & -0.844932 & -0.937091 \\
\hline & & $\mathrm{H}$ & 4.365284 & & 0.040503 & 0.372669 \\
\hline & & $\mathrm{H}$ & 3.500956 & & 0.919324 & -0.894484 \\
\hline & & $\mathrm{H}$ & 2.189331 & & -0.951024 & 1.165411 \\
\hline & & $\mathrm{H}$ & 2.162214 & & 0.796042 & 1.255233 \\
\hline & & $\mathrm{H}$ & 0.915697 & & 0.637331 & -1.162568 \\
\hline & & $\mathrm{H}$ & -0.331369 & & -0.935618 & 1.230072 \\
\hline & & $\mathrm{H}$ & -1.513789 & & 0.037720 & -1.441575 \\
\hline & & $\mathrm{H}$ & -2.918195 & & -0.992564 & 1.077539 \\
\hline & & $\mathrm{H}$ & -2.631670 & & -1.932654 & -0.394714 \\
\hline & & $\mathrm{H}$ & -3.733062 & & -0.539944 & -0.425348 \\
\hline & & $\mathrm{H}$ & -1.047858 & & 2.081966 & -0.065775 \\
\hline & & $\mathrm{H}$ & -2.066215 & & 1.426833 & 1.227740 \\
\hline & & $\mathrm{H}$ & -2.786899 & & 1.848261 & -0.335519 \\
\hline & & RigidRoto & r & & & \\
\hline & & SymmetryF & actor & 0.25 & & \\
\hline & & End & & & & \\
\hline & & Frequencies [1/cm ] & 59 & & & \\
\hline & & 3036.8251 & 3067.9553 & 3076.7642 & 3023.74663027 .5298 & \\
\hline & & 3035.0899 & 2956.0143 & 3017.0501 & 3020.92782941 .7004 & \\
\hline
\end{tabular}


End

\begin{tabular}{|c|c|c|c|c|c|c|c|}
\hline \multirow[t]{41}{*}{ Barrier } & $\begin{array}{l}\text { TS_3_5 } \\
\text { RRHO }\end{array}$ & 3 & 5 & & & & \\
\hline & & Geome & angstrom] & & 22 & & \\
\hline & & & $\mathrm{C}$ & -2.016598 & & 0.043210 & \\
\hline & & & C & -1.012810 & & -1.098968 & \\
\hline & & & C & 0.158573 & & -0.23021 & \\
\hline & & & C & -2.579708 & & 0.653045 & \\
\hline & & & C & 1.182374 & & 0.184510 & \\
\hline & & & C & 2.043321 & & 1.335920 & \\
\hline & & & C & 2.059196 & & -1.00940 & \\
\hline & & & $\mathrm{H}$ & -0.914056 & & -1.44425 & \\
\hline & & & $\mathrm{H}$ & 0.550806 & & -0.42985 & \\
\hline & & & $\mathrm{H}$ & -0.869666 & & 0.717979 & \\
\hline & & & $\mathrm{H}$ & -3.272668 & & -0.03610 & \\
\hline & & & $\mathrm{H}$ & -1.780025 & & 0.877673 & \\
\hline & & & $\mathrm{H}$ & -3.120305 & & 1.580740 & \\
\hline & & & $\mathrm{H}$ & 0.641127 & & 0.519641 & \\
\hline & & & $\mathrm{H}$ & -1.210990 & & -1.96596 & \\
\hline & & & $\mathrm{H}$ & -2.688662 & & -0.02601 & \\
\hline & & & $\mathrm{H}$ & 2.580089 & & 1.031495 & \\
\hline & & & $\mathrm{H}$ & 1.431538 & & 2.207371 & \\
\hline & & & $\mathrm{H}$ & 2.788397 & & 1.636495 & \\
\hline & & & $\mathrm{H}$ & 1.455623 & & -1.84754 & \\
\hline & & & $\mathrm{H}$ & 2.636168 & & -1.35735 & \\
\hline & & & $\mathrm{H}$ & 2.766541 & & -0.73291 & \\
\hline & & Core & RigidRoto & & & & \\
\hline & & & SymmetryF & actor & 0.25 & & \\
\hline & & End & & & & & \\
\hline & & Frequ & $\mathrm{s}[1 / \mathrm{cm}]$ & 59 & & & \\
\hline & & & 3032.9595 & 3033.0375 & 3049.7647 & 3017.6153 & 3026.1815 \\
\hline & & & 3028.9687 & 2985.4341 & 3010.3154 & 3014.1076 & 2941.4984 \\
\hline & & & 2944.6079 & 2962.8319 & 1746.2531 & 2912.4692 & 2920.9855 \\
\hline & & & 1443.2659 & 1451.257 & 1458.5167 & 1435.9774 & 1439.1502 \\
\hline & & & 1440.0662 & 1365.4156 & 1368.7213 & 1432.2724 & 1309.5577 \\
\hline & & & 1342.9091 & 1351.3817 & 1243.472 & 1282.1994 & 1297.0709 \\
\hline & & & 1157.8572 & 1182.2012 & 1219.7112 & 1087.0236 & 1108.5268 \\
\hline & & & 1140.8193 & 978.1574 & 1026.6161 & 1041.3749 & 895.8619 \\
\hline & & & 913.3526 & 944.2602 & 836.2728 & 864.2489 & 886.06 \\
\hline & & & 557.2728 & 718.0917 & 792.5588 & 349.6736 & 390.9039 \\
\hline & & & 446.6989 & 223.6447 & 261.2039 & 316.4932 & 146.0624 \\
\hline & & & 209.9016 & 214.2121 & 69.8994 & 74.2492 & \\
\hline & & Zerol & [ $[\mathrm{kcal} / \mathrm{mo}]$ & & 17.35001 & 016549793 & \\
\hline
\end{tabular}

End

2947.38472948 .39992157 .43112888 .10162912 .507

$1450.11621454 .582 \quad 1459.85281436 .74881439 .5901$

1447.04611366 .43421397 .19041435 .00361339 .5894

1348.21871364 .66851248 .06211285 .45351298 .0338

1163.75321192 .73231230 .07691093 .55311147 .4638

1156.80681016 .12661048 .88041068 .9562893 .4457

$\begin{array}{llllll}928.1651 & 941.0874 & 767.871 & 826.7069 & 883.2804\end{array}$

$\begin{array}{lllll}469.0331 & 652.6996 & 701.3864 & 311.9737 & 377.5123\end{array}$

$\begin{array}{lllll}403.4527 & 231.2806 & 249.0769 & 286.659 & 108.5699\end{array}$

$\begin{array}{llll}149.987 & 220.2891 & 60.5971 & 105.7097\end{array}$

ZeroEnergy [ $\mathrm{kcal} / \mathrm{mol}]$

15.992707117000494

Barrier

TS_3_6

3

6

Geometry[angstrom]

C $\quad-2.080559$

C $\quad-2.043491$

22

C $\quad-1.106042$

C $\quad-0.015873$

C $\quad 1.098087$

C $\quad 1.861331$

C $\quad 2.055953$

1.080021
-0.340500
-1.099649
-0.100095
0.091676
1.383933
-1.104688

0.480431

0.330081

0.782211

$-0.772675$

$-0.245131$

0.266354

$-0.643079$

$-0.705872$

1.782619

0.914898

$-1.275439$

$-1.488094$

$-0.569304$

$-1.143775$

0.963253

1.335686

1.172677

0.516362

$-0.476708$

$-1.004303$

0.221246

$-1.432397$ 


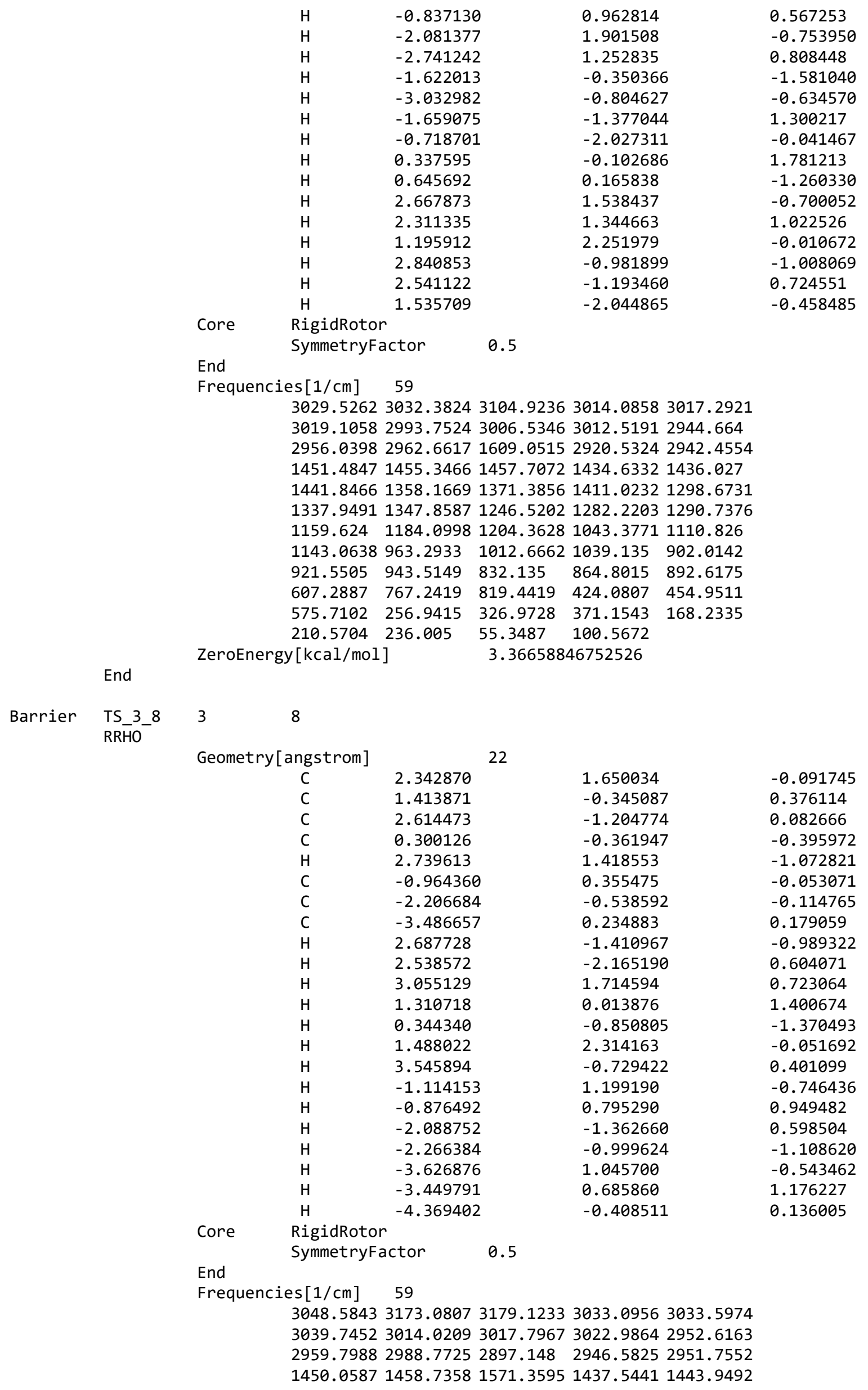


End

$1446.47791374 .4221 \quad 1377.591 \quad 1425.98731340 .6355$

1365.35181367 .84431254 .19951280 .35461293 .7099

1143.80221207 .26591239 .23911031 .56941069 .0503

$1082.0706957 .2619 \quad 1007.3847 \quad 1017.0162846 .6854$

$\begin{array}{lllll}877.1094 & 899.9893 & 711.445 & 735.9095 & 795.4748\end{array}$

$\begin{array}{llllll}486.5259 & 500.4185 & 533.4456 & 272.7824 & 300.2843\end{array}$

$\begin{array}{llllll}383.2291 & 185.5103 & 231.5826 & 263.3979 & 82.5718\end{array}$

$\begin{array}{llll}140.6098 & 165.2695 & 45.8536 & 73.043\end{array}$

ZeroEnergy [ $\mathrm{kcal} / \mathrm{mol}]$

8.780112924018953

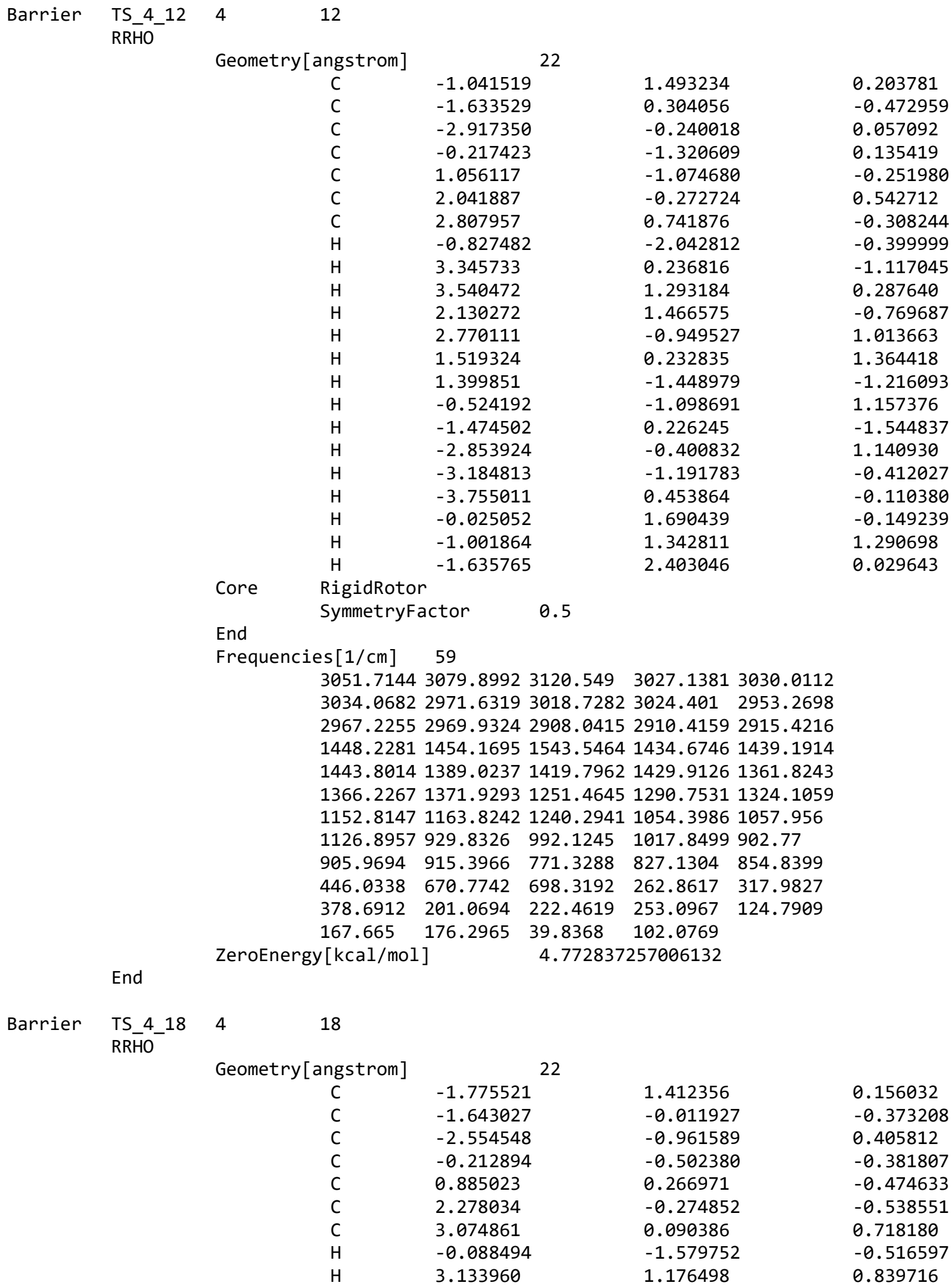




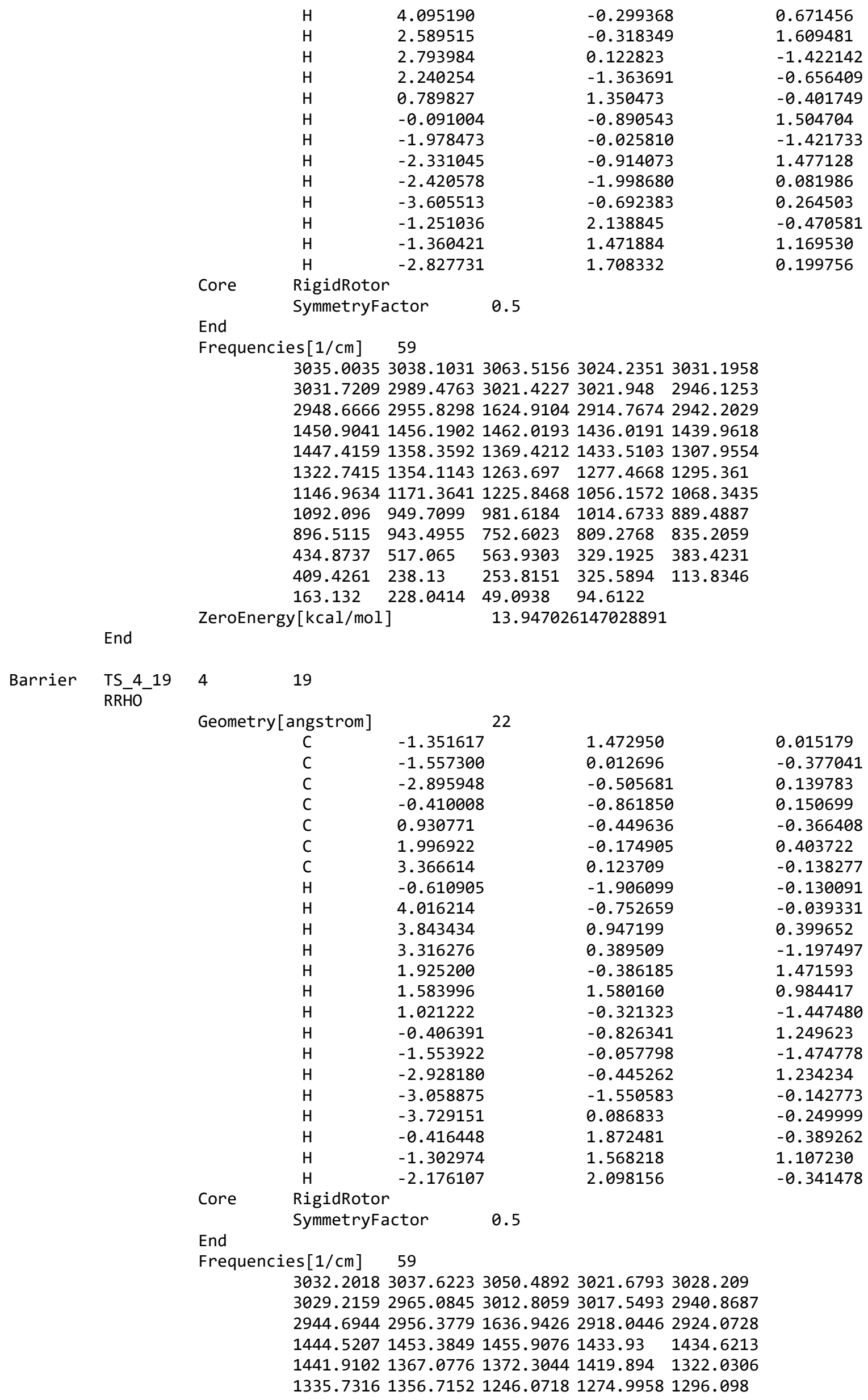


End

1149.59361161 .15391208 .16421042 .05961067 .512

$\begin{array}{lllll}1110.0187 & 950.2202 & 987.1313 & 1035.196 & 897.4835\end{array}$

$\begin{array}{llllll}900.0952 & 938.0046 & 762.0073 & 827.2233 & 866.8008\end{array}$

$\begin{array}{llllll}426.8172 & 495.0668 & 521.4205 & 318.1994 & 389.4205\end{array}$

$\begin{array}{lllll}407.3433 & 244.8662 & 259.9823 & 295.4373 & 119.1631\end{array}$

$\begin{array}{llll}171.1155 & 221.6117 & 74.8386 & 105.7045\end{array}$

ZeroEnergy $[\mathrm{kcal} / \mathrm{mol}] \quad 12.9273232094995$

Barrier

TS_4

4

$\mathrm{RRHO}$

Geometry[angstrom]

C 3.514883

2.201512

C $\quad 2.201512$

C $\quad-0.324561$

C $\quad-1.605131$

C $\quad-2.819720$

$\mathrm{H} \quad 1.445804$

$\mathrm{H} \quad 3.758890$

H $\quad 3.490330$

$\mathrm{H} \quad 4.334695$

$\mathrm{H} \quad 1.030324$

$\mathrm{H} \quad-0.305047$

$\mathrm{H} \quad-0.366843$

$\mathrm{H} \quad-1.524015$

$\mathrm{H} \quad-3.743897$

$\mathrm{H} \quad-2.730887$

$\mathrm{H} \quad-2.919460$

C $\quad-1.767390$

H 2.141527

$\mathrm{H} \quad-2.676250$

H $\quad-1.843052$

H $\quad-0.914327$

Core RigidRotor

End

$$
\text { SymmetryFactor } \quad 0.25
$$

Frequencies [1/cm ] 59

3033.70843073 .00123098 .17733017 .56133030 .5183 $3033.363 \quad 2945.34792976 .50243013 .98112915 .5764$ 2932.69442942 .25992162 .05822877 .42812908 .8709 1447.16751451 .71781458 .30371433 .15921436 .5646 $1441.80691370 .83271390 .96 \quad 1424.16111336 .8859$ $1354.461 \quad 1365.75261238 .60361286 .97971321 .1471$ $1170.20581193 .48171229 .704 \quad 1096.07221136 .5361$ $\begin{array}{llllll}1162.763 & 998.7962 & 1036.8239 & 1053.5666 & 896.7513\end{array}$ $\begin{array}{lllll}934.7074 & 944.6224 & 813.3178 & 862.1808 & 889.6244\end{array}$ $\begin{array}{llllll}452.0796 & 620.9356 & 701.8962 & 297.5308 & 384.6346\end{array}$ $\begin{array}{lllll}415.2508 & 212.1276 & 237.8932 & 291.1818 & 111.0704\end{array}$ $\begin{array}{llll}178.2643 & 197.4871 & 65.4273 & 75.8903\end{array}$

End ZeroEnergy [kcal/mol]

\subsection{5}

0.039262

$-0.637832$

0.020444

$-0.836167$

0.878514

0.944748

0.148257

0.393984

$-1.694538$

$-0.665489$

$-0.391158$

$-1.845229$

$-0.929114$

1.430880

1.906551

1.394512

2.065600
-0.206174
0.473592
-0.275415
0.154732
-0.368823
-0.022751
0.576260
-0.879233
-0.825316
0.507774
-1.299231
-0.163807
1.255490
-1.463850
-0.403824
-0.437262
1.065603
0.191240
1.358593
-0.190055
1.285354
-0.064903 $\begin{array}{llll}\text { Barrier } & \text { TS_4_6 } & 4 & 6 \\ \text { RRHO } & & \end{array}$

Geometry [angstrom]

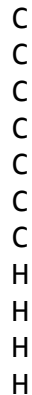

$$
\begin{aligned}
& -2.798734 \\
& -2.405674 \\
& -1.029889 \\
& 0.139090 \\
& 1.400897 \\
& 2.474742 \\
& 1.934485 \\
& -1.547043 \\
& -2.754377 \\
& -3.578906 \\
& -2.365430
\end{aligned}
$$

22

$$
\begin{aligned}
& -0.407866 \\
& 0.101310 \\
& 0.549463 \\
& -0.330261 \\
& -0.093268 \\
& -1.111291 \\
& 1.326856 \\
& 0.138706 \\
& -1.480394 \\
& 0.120460 \\
& -0.670214
\end{aligned}
$$

-0.749319
0.632073
0.142981
0.489101
-0.344996
0.030821
-0.167668
-1.083079
-0.922690
-1.291195
1.405907 
End

$\begin{array}{llll}\mathrm{H} & -0.856730 & 1.625215 & 0.109713 \\ \mathrm{H} & -0.172137 & -1.380396 & 0.380932 \\ \mathrm{H} & 0.394329 & -0.205055 & 1.556565 \\ \mathrm{H} & 1.133175 & -0.236015 & -1.401670 \\ \mathrm{H} & 2.117516 & -2.137585 & -0.100502 \\ \mathrm{H} & 3.375444 & -0.983752 & -0.577322 \\ \mathrm{H} & 2.762742 & -0.989086 & 1.082011 \\ \mathrm{H} & 2.144175 & 1.522950 & 0.891299 \\ \mathrm{H} & 2.867350 & 1.465829 & -0.722837 \\ \mathrm{H} & 1.224888 & 2.081940 & -0.516368 \\ \mathrm{H} & -3.034488 & 0.917738 & 0.991279\end{array}$

$$
\begin{array}{ll}
\text { Core } & \begin{array}{l}
\text { RigidRotor } \\
\text { SymmetryFactor }
\end{array} \\
\text { End } & 0.5
\end{array}
$$$$
\text { Frequencies }[1 / \mathrm{cm}] \quad 59
$$$$
3037.34843051 .71573134 .50173028 .12713028 .386
$$$$
3032.10362984 .87463012 .81423016 .33432937 .7199
$$$$
2941.66892945 .13781751 .36892876 .83342926 .9272
$$$$
1444.08071453 .61181460 .66891419 .61261437 .8034
$$$$
1443.39851356 .79071371 .39951382 .98411303 .807
$$$$
1323.29151351 .02181223 .1932 \quad 1242.813 \quad 1295.2755
$$$$
1162.28321184 .087 \quad 1211.997 \quad 1059.91321116 .0967
$$$$
\begin{array}{lllll}
1140.4361 & 959.3954 & 992.1358 & 1040.333 & 898.901
\end{array}
$$$$
\begin{array}{llllll}
914.6719 & 943.7672 & 816.5761 & 885.3365 & 894.3305
\end{array}
$$$$
\begin{array}{lllll}
626.2678 & 717.8349 & 779.6894 & 368.7553 & 414.6985
\end{array}
$$$$
\begin{array}{lllll}
441.5809 & 243.6042 & 302.4699 & 348.0433 & 114.3819
\end{array}
$$$$
\begin{array}{llll}
172.2385 & 220.1876 & 45.1609 & 66.1253
\end{array}
$$$$
\text { ZeroEnergy [kcal/mol] }
$$$$
19.04993340102208
$$

\begin{tabular}{|c|c|c|c|c|}
\hline Geometry[angstrom] & & 22 & & \\
\hline $\mathrm{C}$ & -0.854152 & & 1.424332 & \\
\hline C & -1.443793 & & 0.017792 & \\
\hline C & -2.775121 & & -0.036702 & \\
\hline C & -0.461818 & & -1.007407 & \\
\hline C & 0.839366 & & -1.077427 & \\
\hline C & 2.053738 & & -0.985504 & \\
\hline C & 2.678058 & & 1.183553 & \\
\hline $\mathrm{H}$ & -0.947629 & & -1.994806 & \\
\hline $\mathrm{H}$ & 2.636045 & & 1.494840 & \\
\hline $\mathrm{H}$ & 3.660608 & & 1.032086 & \\
\hline $\mathrm{H}$ & 1.900198 & & 1.554287 & \\
\hline $\mathrm{H}$ & 2.957676 & & -1.182910 & \\
\hline $\mathrm{H}$ & 2.138450 & & -1.053725 & \\
\hline $\mathrm{H}$ & 0.780800 & & -1.158675 & \\
\hline $\mathrm{H}$ & -0.274868 & & -0.764343 & \\
\hline $\mathrm{H}$ & -1.617777 & & -0.251183 & \\
\hline $\mathrm{H}$ & -2.631246 & & 0.225699 & \\
\hline $\mathrm{H}$ & $-3.21541 e$ & & -1.038065 & \\
\hline $\mathrm{H}$ & -3.496626 & & 0.669312 & \\
\hline $\mathrm{H}$ & 0.087528 & & 1.483537 & \\
\hline $\mathrm{H}$ & -0.648143 & & 1.711734 & \\
\hline $\mathrm{H}$ & -1.547279 & & 2.160382 & \\
\hline \multicolumn{5}{|c|}{ RigidRotor } \\
\hline \multicolumn{2}{|c|}{ SymmetryFactor } & 0.5 & & \\
\hline \multicolumn{5}{|l|}{ End } \\
\hline Frequencies $[1 / \mathrm{cm}]$ & 59 & & & \\
\hline 3140.2737 & 3181.1382 & 3188.7447 & 3033.0457 & 3049.9069 \\
\hline 3053.5985 & 3016.1228 & 3021.8863 & 3027.578 & 2943.7483 \\
\hline 2956.6274 & 3011.6092 & 2910.1698 & 2923.2864 & 2939.3741 \\
\hline 1452.0793 & 1457.4585 & 1546.7781 & 1423.3228 & 1436.781 \\
\hline 1441.3688 & 1373.8527 & 1378.6989 & 1392.882 & 1325.8038 \\
\hline 1354.6348 & 1367.9379 & 1248.1427 & 1255.9817 & 1322.4643 \\
\hline 1159.6075 & 1166.7087 & 1217.5648 & 984.5286 & 1033.1158 \\
\hline 1107.6845 & 929.6459 & 942.051 & 946.6325 & 857.1309 \\
\hline
\end{tabular}

Barrier $\quad$ TS_4_9

-0.304199
-0.351293
0.391692
0.234636
-0.500276
0.087718
0.273546
0.223707
-0.762553
0.704317
0.928899
-0.479386
1.169649
-1.586052
1.291089
-1.403597
1.446756
0.353581
-0.030716
-0.858491
0.734983
-0.723128 
End

\begin{tabular}{lllll}
893.1716 & 900.5727 & 674.8855 & 758.3499 & 815.257 \\
425.274 & 489.8994 & 492.3375 & 329.7491 & 378.9109 \\
422.9686 & 222.2221 & 269.5411 & 280.4976 & 128.0716 \\
161.5847 & 180.7459 & 58.8398 & 94.4617 & \\
$y[\mathrm{kcal} / \mathrm{mol}]$ & \multicolumn{3}{c}{8.384781939018314} &
\end{tabular}

ZeroEnergy [kcal/mol] $\quad 8.384781939018314$

$\begin{array}{llll}\text { Barrier } & \text { TS_5_14 } & 5 & 14 \\ \text { RRHO } & & \end{array}$

Geometry[an

22

$-1.303248$

$-0.388199$

$-0.342071$

0.041490

$-0.692180$

0.406226

0.278605

0.007431

$-0.163544$

0.225053

1.046913

$-1.686064$

$-0.659351$

0.616810

1.358322

$-0.169523$

1.293995

$-0.477699$

$-1.285599$

$-0.987634$

0.047585

0.743359

0.492372

$-0.788372$

0.907566

Core RigidRotor

SymmetryFactor $\quad 0.5$

End

Frequencies [1/cm] 59

3073.38053128 .00523133 .66493034 .74973035 .1402

$\begin{array}{llllll}3036.6857 & 3020.117 & 3026.1997 & 3031.8578 & 2947.5155\end{array}$

2989.50613015 .20872912 .64072934 .66232941 .6551

1452.52271459 .76131551 .01181437 .58721439 .3754

$1442.33431388 .04321415 .38 \quad 1428.20541348 .4443$

$1354.61761364 .544 \quad 1249.64891289 .22791319 .9746$

$\begin{array}{lllll}1154.5618 & 1161.4221 & 1170.812 & 979.2729 & 1017.8156\end{array}$

$1095.4239930 .5391 \quad 944.1096 \quad 959.4008 \quad 894.1613$

$\begin{array}{lllll}899.1147 & 906.1196 & 756.767 & 807.2943 & 889.8024\end{array}$

$\begin{array}{lllll}415.7416 & 539.3123 & 657.0535 & 339.2427 & 366.2523\end{array}$

$\begin{array}{lllll}409.9801 & 220.5034 & 251.1934 & 267.7964 & 103.4215\end{array}$

$\begin{array}{llll}131.7343 & 153.6991 & 41.755 & 61.0169\end{array}$

ZeroEnergy [ $\mathrm{kcal} / \mathrm{mol}]$

7.712719264502959

End

Barrier

TS_5_19 5

RRHO

19

Geometry [angstrom]

$\mathrm{C}$
$\mathrm{C}$
$\mathrm{C}$
$\mathrm{C}$
$\mathrm{C}$
$\mathrm{C}$
$\mathrm{C}$
$\mathrm{H}$
$\mathrm{H}$
$\mathrm{H}$
$\mathrm{H}$
$\mathrm{H}$
$\mathrm{H}$
22

-2.154035
-0.988506
0.321077
-3.474766
1.523586
2.766779
1.762952
-0.723996
0.568745
0.193833
-4.202959
-3.379611
-3.898461

$-0.510752$

0.102376

$-0.623813$

0.182354

0.004387

$-0.847901$

1.439104

0.969095

$-0.676229$

$-1.659240$

$-0.296499$

1.231155

0.150155
0.040582

$-0.228437$

$-0.371272$

0.087566

0.345361

0.104657

$-0.117322$

1.459992

$-1.443586$

$-0.031675$

$-0.575908$

$-0.205945$

1.098491 


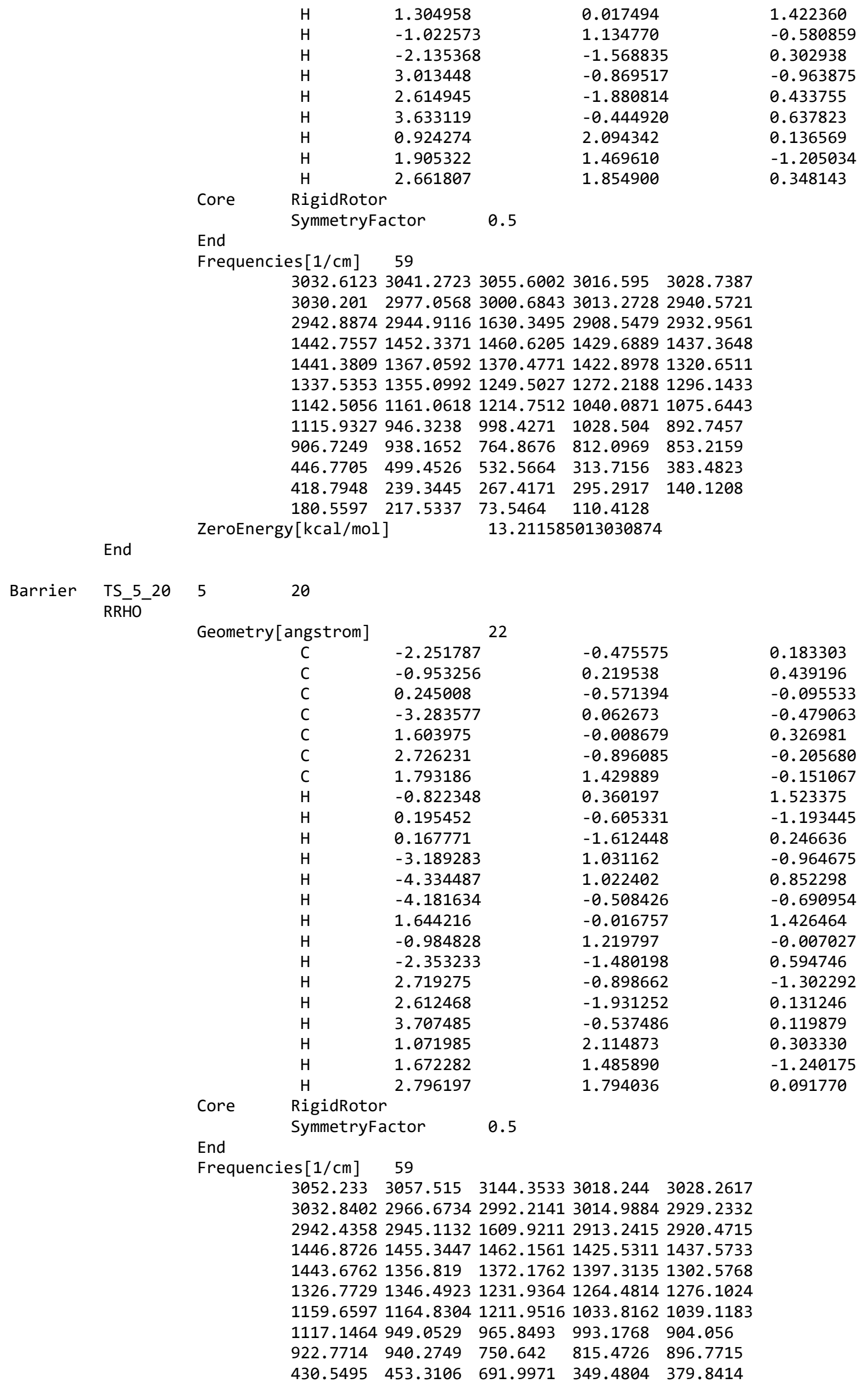


End

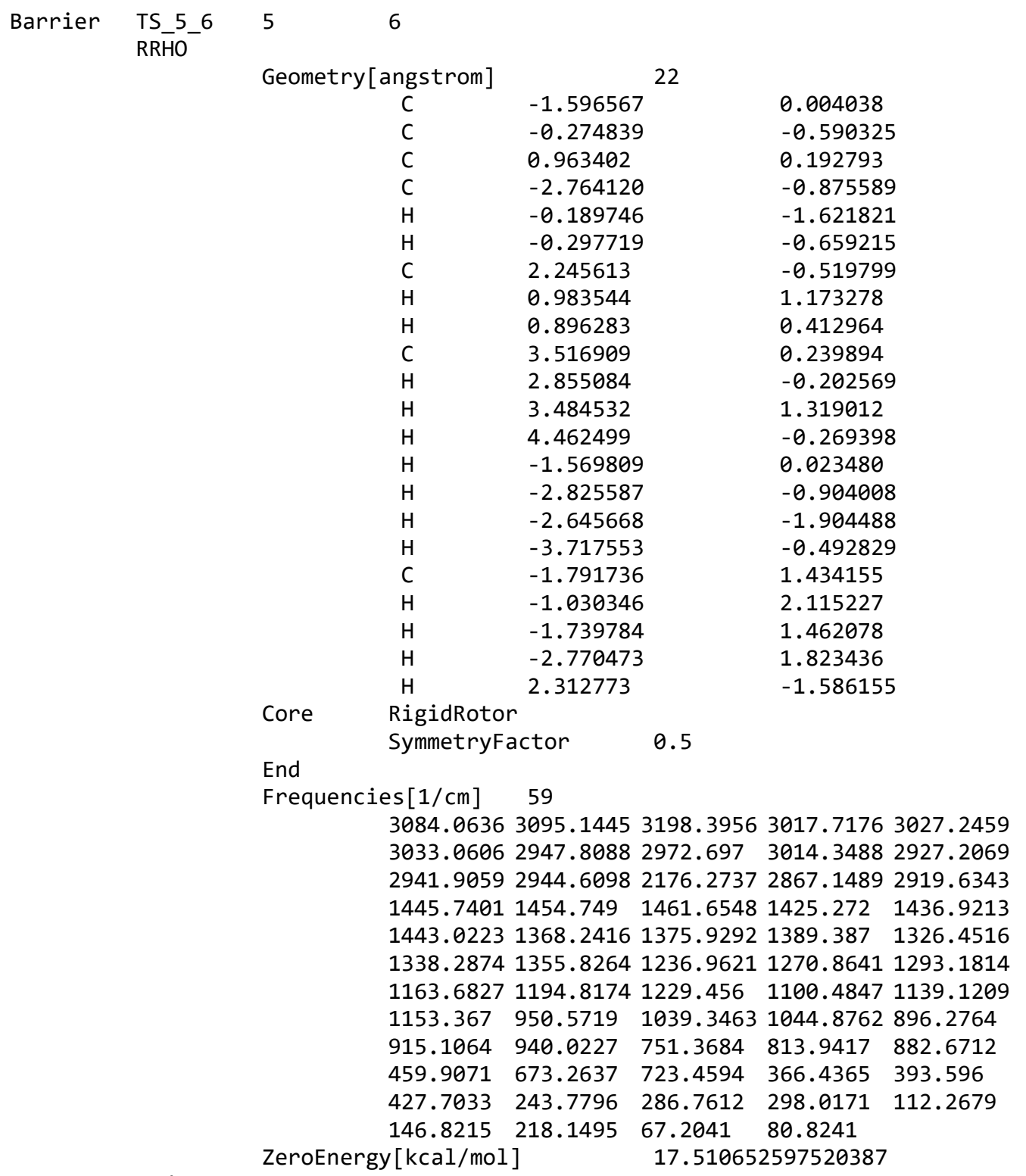

Barrier TS_6_15 $6 \quad 15$

RRHO

Geometry[angstrom]

C

C

$\mathrm{H}$

$\mathrm{H}$

$\mathrm{H}$

C

$\mathrm{C}$

H

$\mathrm{H}$

$\mathrm{H}$

$\mathrm{H}$
$409.1094 \quad 243.6271$

$50.5278 \quad 220.7796$

248.9009

64.018
105.9457

0.342500

0.148162

$-0.282780$

0.097593

$-0.220620$

1.245598

0.003602

0.212126

$-1.363912$

0.077986

1.102442

$-0.000441$

$-0.045498$

$-1.442218$

1.192172

$-0.256628$

$-0.279326$

0.158147

$-0.232408$

1.253881

$-0.138984$

$-0.187436$ 


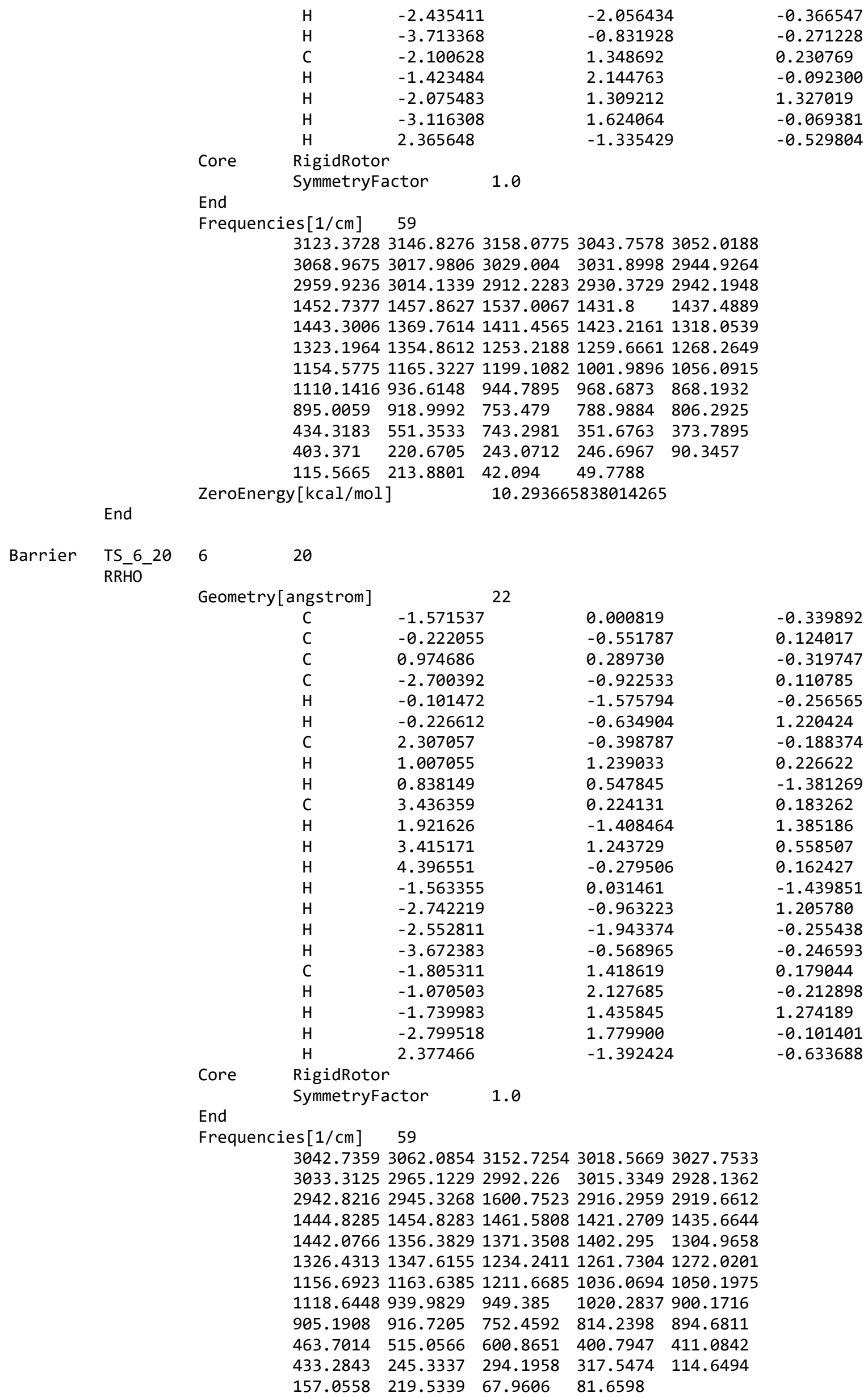




\section{Input file for $M E S S$ for case 3}

TemperatureList [K]

PressureList [torr]

$0.1110 \quad 1 \mathrm{E} 2 \quad 1 \mathrm{E} 4$

EnergyStepOverTemperature

ExcessEnergyOverTemperature

ModelEnergyLimit [ $\mathrm{kcal} / \mathrm{mol}]$

CalculationMethod

ChemicalEigenvalueMax

ChemicalEigenvalueMin

EigenvalueOutput

Model

EnergyRelaxation

Exponential

Factor $[1 / \mathrm{cm}]$

Power

End

CollisionFrequency

LennardJones Epsilons [1/cm] Sigmas [angstrom] End Masses [amu ]

$\begin{array}{ll}\text { Well } & 1 \\ & \text { Species } \\ & \text { RRHO }\end{array}$

400. 700

1E-7 $\quad 3 \mathrm{E}-7 \quad 5 \mathrm{E}-7 \quad 7 \mathrm{E}-7 \quad 1 \mathrm{E}-6 \quad 3 \mathrm{E}-6 \quad 5 \mathrm{E}-6 \quad 7 \mathrm{E}-6 \quad 1 \mathrm{E}-5 \quad 3 \mathrm{E}-5 \quad 5 \mathrm{E}-5 \quad 1 \mathrm{E}-4 \quad 1 \mathrm{E}-3 \quad 1 \mathrm{E}-2$

.2

30

400

direct

$$
\begin{aligned}
& .4 \\
& 1 E-7 \\
& \quad \text { eval .out }
\end{aligned}
$$

130.

0

$$
\begin{array}{cc}
124.342 & 197.807 \\
3.655 & 7.61 \\
40 & 99 \quad ! \mathrm{Ar}
\end{array}
$$

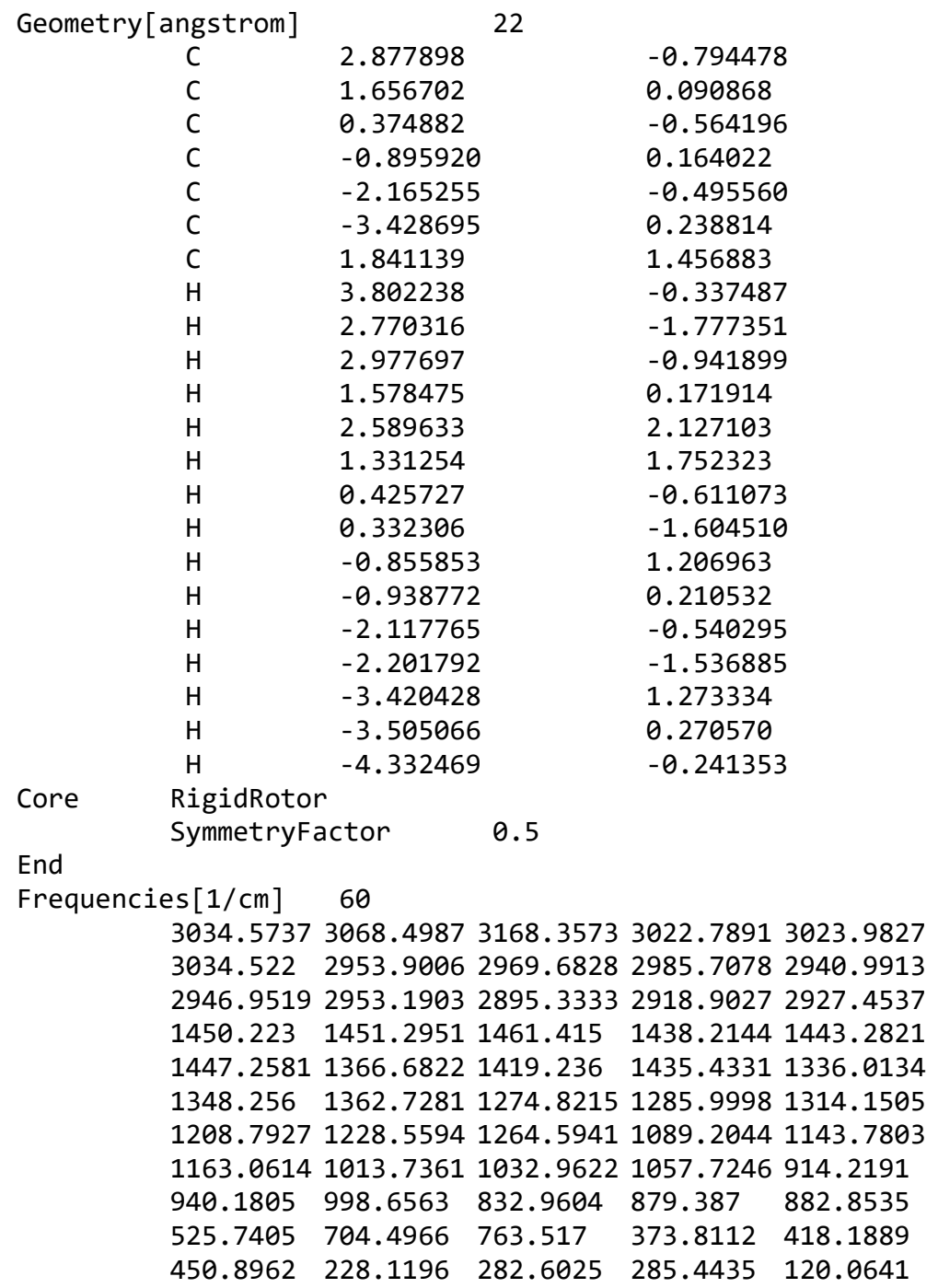

0.027662

0.333186

$-0.186344$

0.235953

$-0.292788$

0.141314

$-0.232058$

0.391760

0.498471

$-1.052897$

1.429911

0.172026

$-1.142041$

$-1.284847$

0.163433

$-0.107692$

1.333670

$-1.388538$

0.052687

$-0.217358$

1.233171

$-0.243309$ 
End

$\begin{array}{lllll}154.2477 & 223.7787 & 55.6091 & 75.2174 & 92.5654\end{array}$

End

ZeroEnergy [kcal/mol]

$-19.92656417248466$

$\begin{array}{cc}\text { Bimolecular } & 13 \\ \text { Fragment } & 13 \_m \\ \text { RRHO } & \end{array}$

Geometry[angstrom]

$\begin{array}{llll}C & 1.227078 & -0.161999 & 0.000117\end{array}$

9

$\begin{array}{llll}\mathrm{H} & 1.800272 & 0.148233 & -0.879810\end{array}$

$\begin{array}{llll}\mathrm{H} & 1.801086 & 0.150329 & 0.878859\end{array}$

$\begin{array}{llll}\mathrm{H} & 1.162752 & -1.253068 & 0.001438\end{array}$

$\begin{array}{llll}C & -0.135226 & 0.458027 & -0.000447\end{array}$

$\begin{array}{llll}\mathrm{H} & -0.173468 & 1.547313 & 0.000845\end{array}$

$\begin{array}{llll}\text { C } & -1.271722 & -0.222539 & -0.000082\end{array}$

$\begin{array}{llll}\mathrm{H} & -2.235213 & 0.276517 & 0.000960\end{array}$

H $\quad-1.276207 \quad-1.310257 \quad 0.000182$

Core RigidRotor

End

SymmetryFactor $\quad 1.0$

Frequencies [1/cm ] 21

$\begin{array}{llllll}3054.8273 & 3063.0255 & 3145.1153 & 2953.685 & 3014.79\end{array}$

3038.46721432 .52831445 .10041680 .74971285 .0092

$1361.18761402 .9944998 .6095 \quad 1045.34991156 .6318$

$\begin{array}{lllll}909.3304 & 913.4013 & 937.4509 & 199.9229 & 410.9365\end{array}$

576.2202

ZeroEnergy $[\mathrm{kcal} / \mathrm{mol}] \quad 0.0$

End

Fragment 13_r

RRHO

End

Geometry[angstrom]

C $\quad 1.525216$

C $\quad 0.119477$

C 0.000000

C $\quad-1.454980$

$\mathrm{H} \quad 2.073247$

$\mathrm{H} \quad 2.073247$

$\mathrm{H} \quad-0.413747$

$\mathrm{H} \quad-0.413747$

H $\quad 0.522215$

$\mathrm{H} \quad 0.522215$

$\mathrm{H} \quad-1.981255$

$\mathrm{H} \quad-1.981255$

$\mathrm{H} \quad-1.539200$

Core RigidRotor

End

SymmetryFactor

1.0
13

-1.283090
-0.803506
0.733143
1.187427
-1.396263
-1.396263
-1.186692
-1.186692
1.132190
1.132190
0.810169
0.810169
2.277356

0.000000

0.000000

0.000000

0.000000

0.926438

$-0.926438$

0.880040

$-0.880040$

0.877378

$-0.877378$

0.882981

$-0.882981$

0.000000
End

Frequencies [1/cm ] 33

3036.32173078 .30573175 .49552970 .37892995 .4441

3026.18462930 .64122952 .25392955 .38691444 .5112

1448.54691458 .34131362 .94461420 .94661440 .3596

$1249.38541283 .204 \quad 1317.9396 \quad 1067.5348 \quad 1159.7583$

$1240.9046914 .781 \quad 1016.66671054 .1714706 .0651$

$\begin{array}{llllll}769.3479 & 855.6594 & 246.1997 & 387.6417 & 519.4796\end{array}$

$\begin{array}{lll}37.8089 & 120.798 \quad 228.4641\end{array}$

ZeroEnergy [kcal/mol]

GroundEnergy [kcal/mol]

1.7676942614957243

Bimolecular

16

Fragment 16_m

RRHO

Geometry[angstrom]

21

C $\quad-2.931223$

$-0.753819$

0.000129 


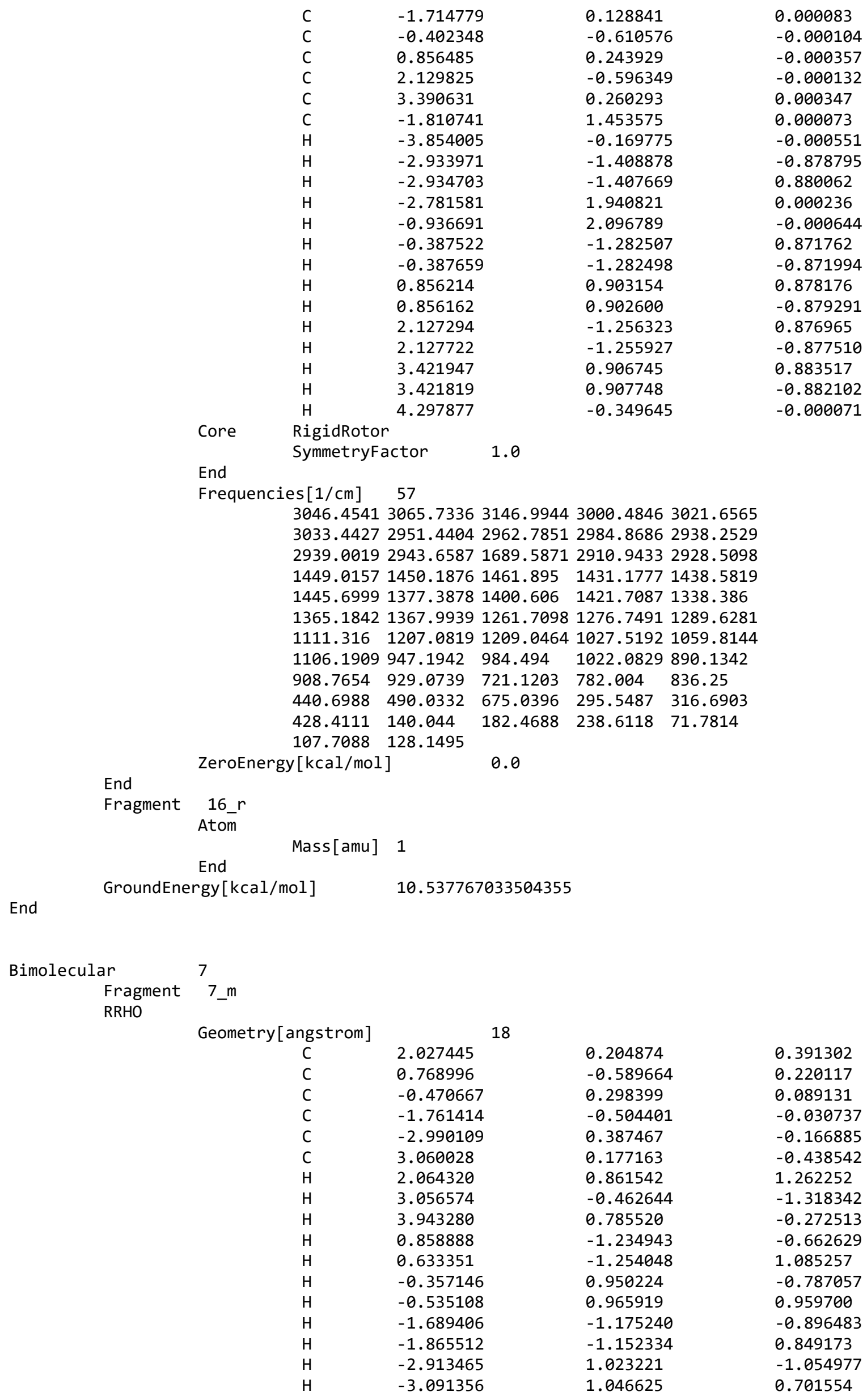




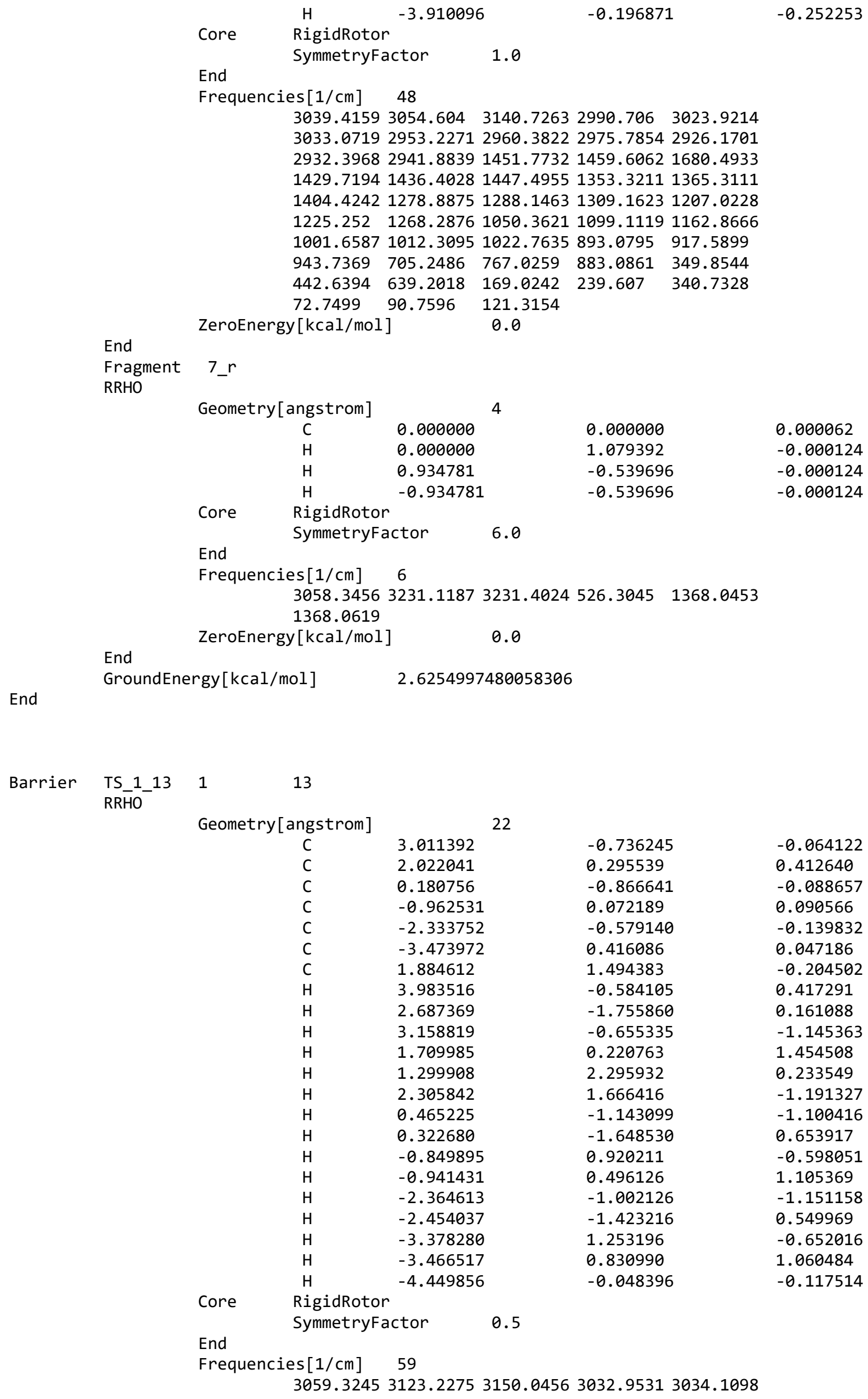


End

3047.10333018 .63683023 .53093029 .69852952 .9595 2962.79382988 .25562917 .65572947 .11452952 .0536 1451.34361458 .44641542 .13161436 .09151443 .8032 1446.32211390 .81381420 .93971434 .48861328 .9358 $1357.457 \quad 1364.29851248 .16231257 .61681284 .7043$

1146.48151168 .33541242 .34071022 .21251062 .4697

$\begin{array}{lllll}1085.1471 & 927.8195 & 975.293 & 1014.0825 & 868.822\end{array}$

$\begin{array}{lllll}895.8943 & 907.8554 & 777.5329 & 781.794 & 837.3792\end{array}$

$\begin{array}{lllll}540.8591 & 546.3868 & 707.3431 & 305.322 & 400.7278\end{array}$

$\begin{array}{llllll}410.9918 & 216.1383 & 235.9629 & 247.9283 & 91.2369\end{array}$

$\begin{array}{llll}124.822 & 163.8785 & 46.7074 & 58.4824\end{array}$

ZeroEnergy [ $\mathrm{kcal} / \mathrm{mol}] \quad 9.603405388009186$

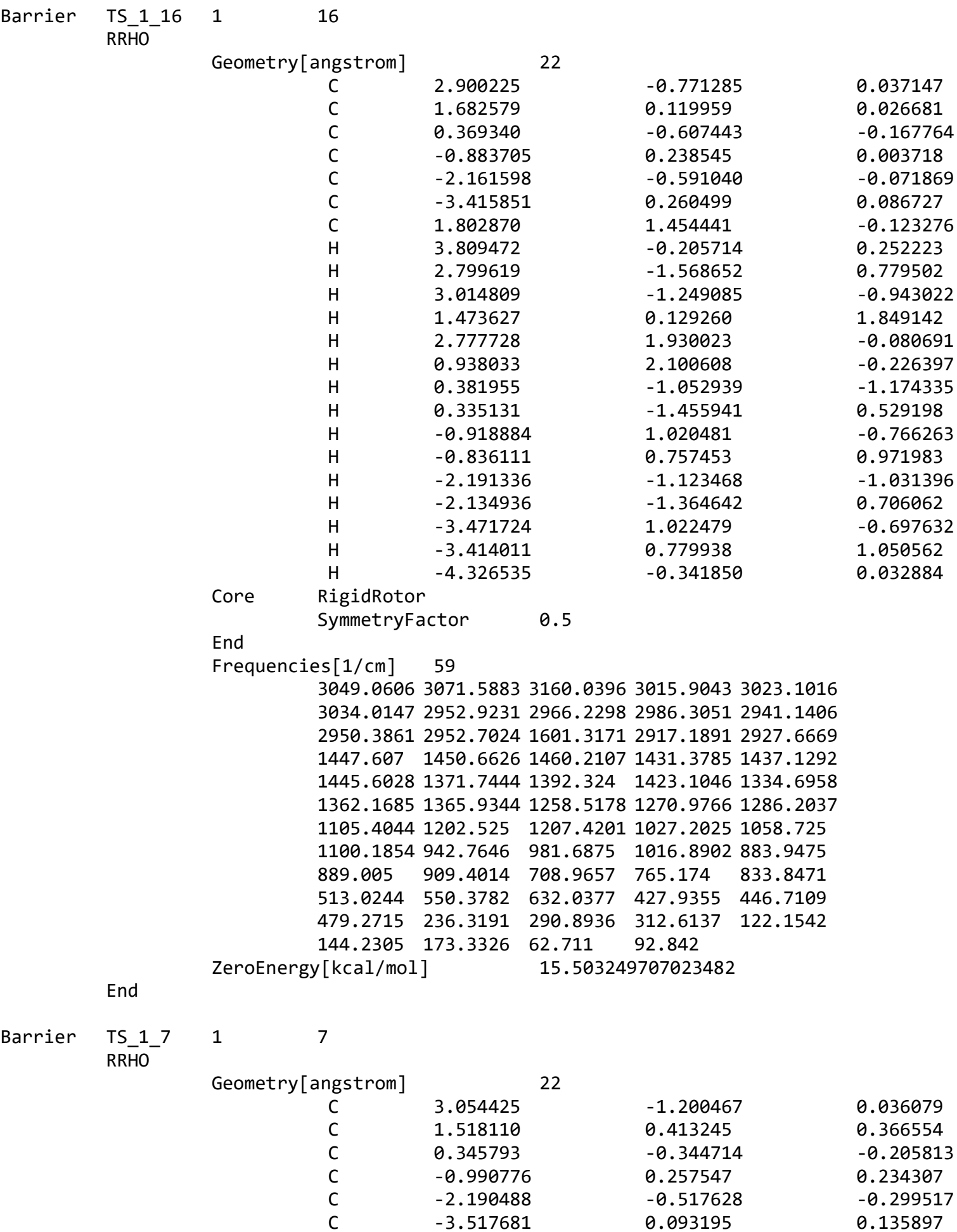




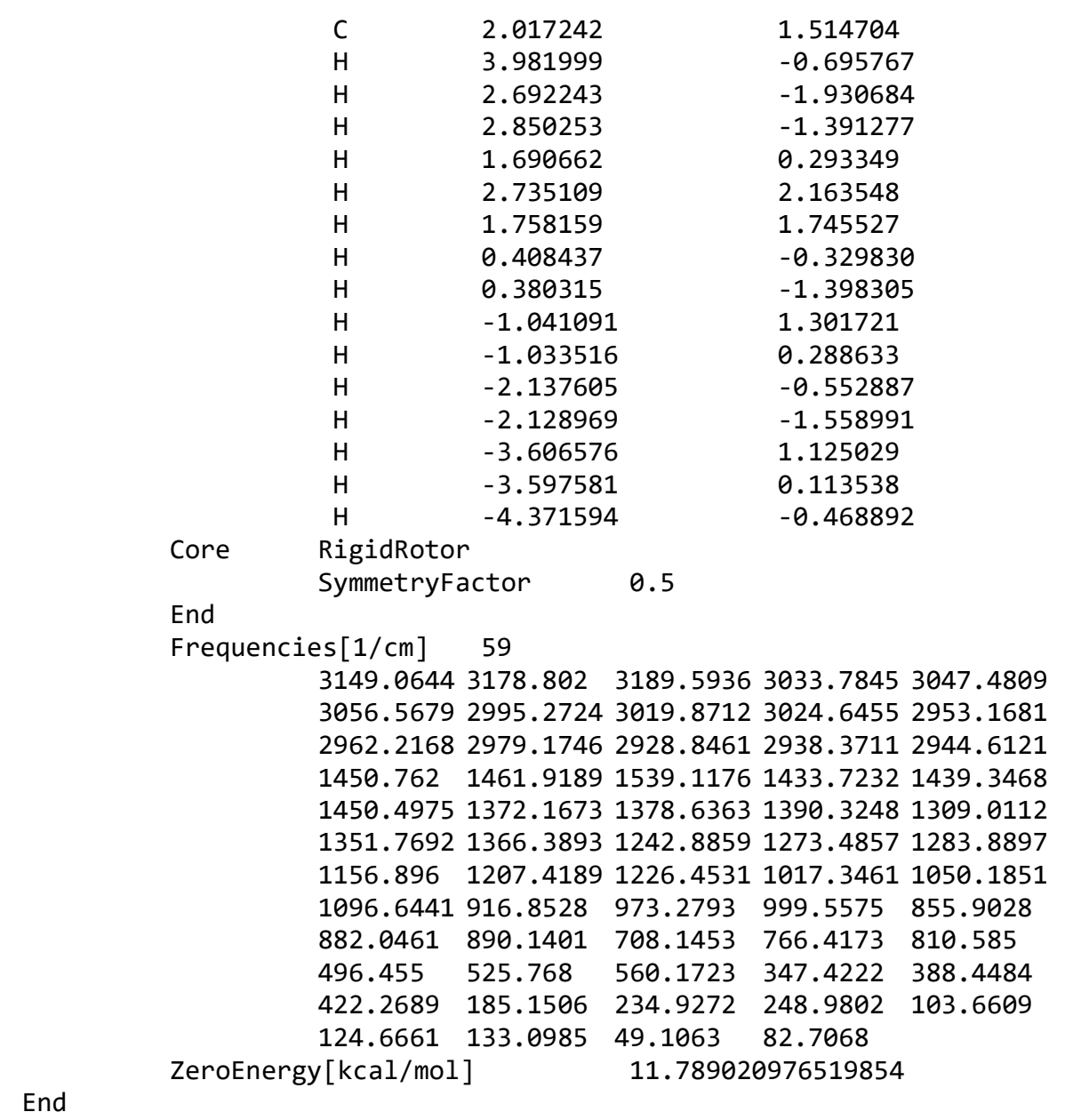

$-0.242751$

0.272194

0.750667

$-1.010251$

1.436256

0.246452

$-1.272970$

$-1.302267$

0.096729

$-0.101033$

1.332060

$-1.395379$

0.042079

$-0.219234$

1.227729

$-0.251566$ 


\section{Input file for MESS for case 4}

TemperatureList [K]

PressureList[torr]

1E6 1E9 1E10

EnergyStepOverTemperature

ExcessEnergyOverTemperature

ModelEnergyLimit [ $\mathrm{kcal} / \mathrm{mol}]$

CalculationMethod

ChemicalEigenvalueMax

ChemicalEigenvalueMin

EigenvalueOutput

Model

EnergyRelaxation

Exponential

Factor $[1 / \mathrm{cm}]$

Power

End

CollisionFrequency

LennardJones

Epsilons [1/cm]

Sigmas [angstrom]

End

Masses [amu ]

$\begin{array}{ll}\text { Well } & 1 \\ & \text { Species } \\ & \text { RRHO }\end{array}$

400.

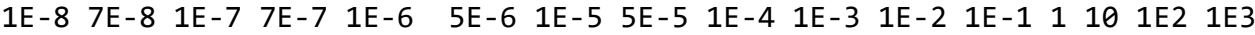

.2

30

400

direct

.2

$1 \mathrm{E}-7$

eval.out

130.

0

$124.342 \quad 197.807$

3.6557 .61

$4099 ! \mathrm{Ar}$

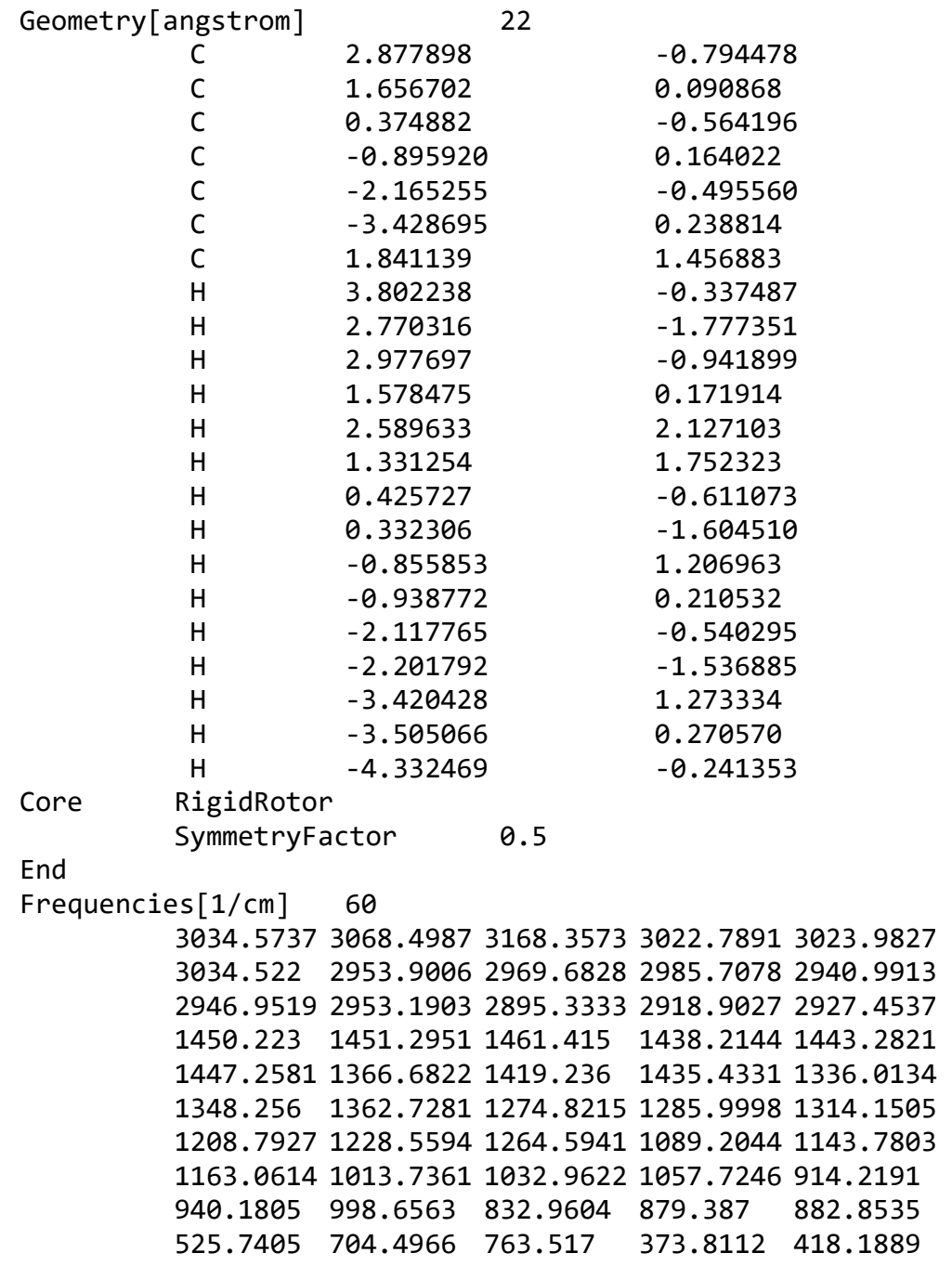

0.027662

0.333186

$-0.186344$

0.235953

$-0.292788$

0.141314

$-0.232058$

0.391760

0.498471

$-1.052897$

1.429911

0.172026

$-1.142041$

$-1.284847$

0.163433

$-0.107692$

1.333670

$-1.388538$

0.052687

$-0.217358$

1.233171

$-0.243309$ 
End

$\begin{array}{lllll}450.8962 & 228.1196 & 282.6025 & 285.4435 & 120.0641\end{array}$

$\begin{array}{lllll}154.2477 & 223.7787 & 55.6091 & 75.2174 & 92.5654\end{array}$

ZeroEnergy [kcal/mol] $\quad-19.92656417248466$

End

Well 2

Species

RRHO

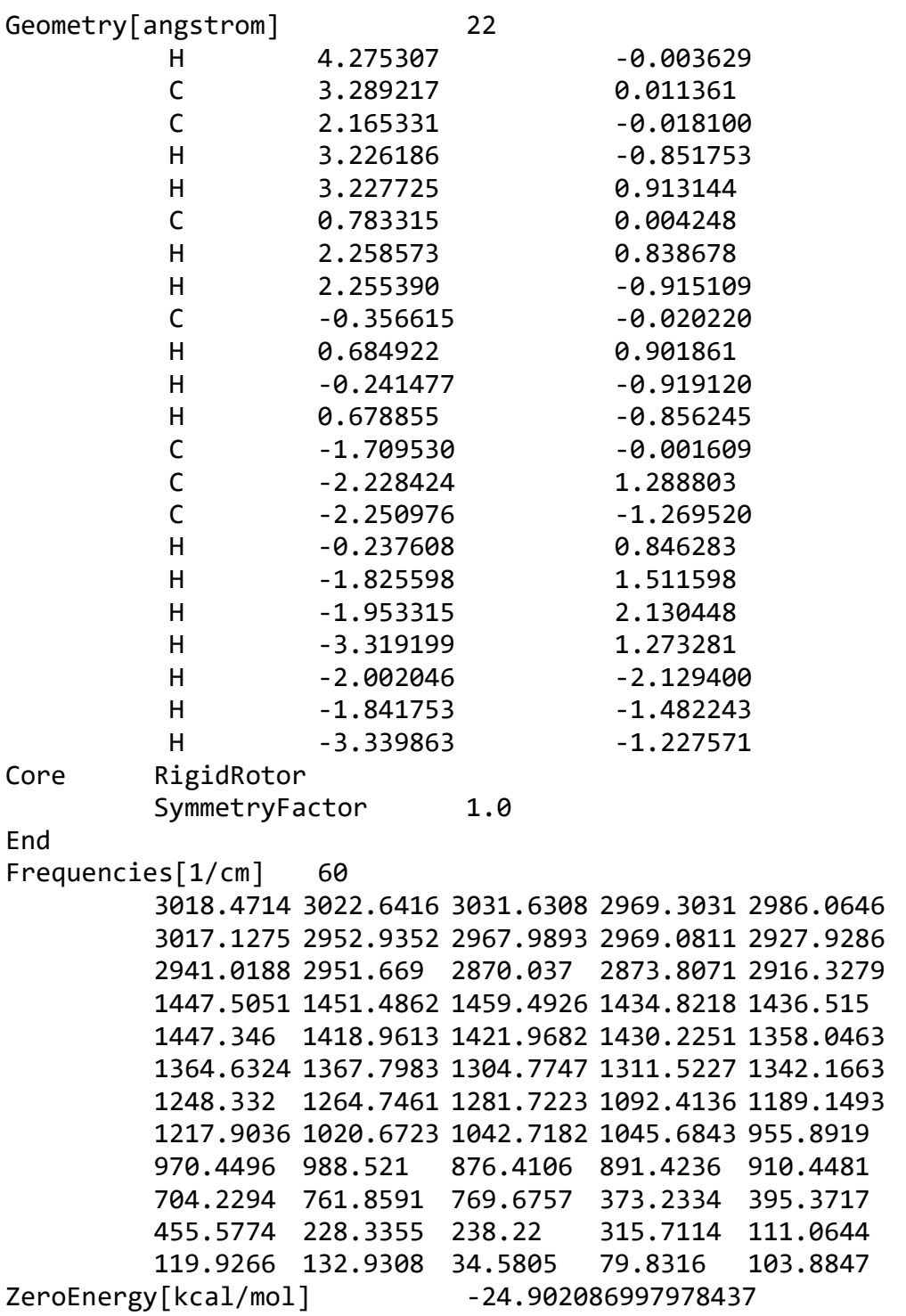

End

End .902086997978437

Well 6

Species RRHO

$\begin{array}{cl}\text { Geometry[angstrom] } & \\ \text { C } & -1.570543 \\ \text { C } & -0.263497 \\ \text { C } & 0.987746 \\ \text { C } & -2.746698 \\ \text { H } & -0.163361 \\ \text { H } & -0.332205 \\ \text { C } & 2.274107 \\ \text { H } & 0.970629 \\ \text { H } & 1.000006 \\ \text { C } & 3.502665 \\ \text { H } & 2.223621\end{array}$

22

$\begin{array}{ll}0.024556 & -0.357283 \\ -0.583827 & 0.156491 \\ 0.214357 & -0.192748 \\ -0.884701 & -0.009564 \\ -1.601148 & -0.247578 \\ -0.696195 & 1.249624 \\ -0.515603 & 0.191244 \\ 1.187031 & 0.315006 \\ 0.428821 & -1.270347 \\ 0.268361 & -0.090837 \\ -0.772729 & 1.264770\end{array}$

$-0.077987$

$-0.550075$

0.479556

$-1.220911$

$-1.168118$

$-0.163744$

1.159347

1.105759

0.870971

$-0.789977$

1.492884

$-0.839185$

0.241551

$-0.291512$

$-0.322016$

1.536912

$-1.295319$

0.353123

$-0.393225$

0.309023

$-1.325478$

$-0.435225$ 


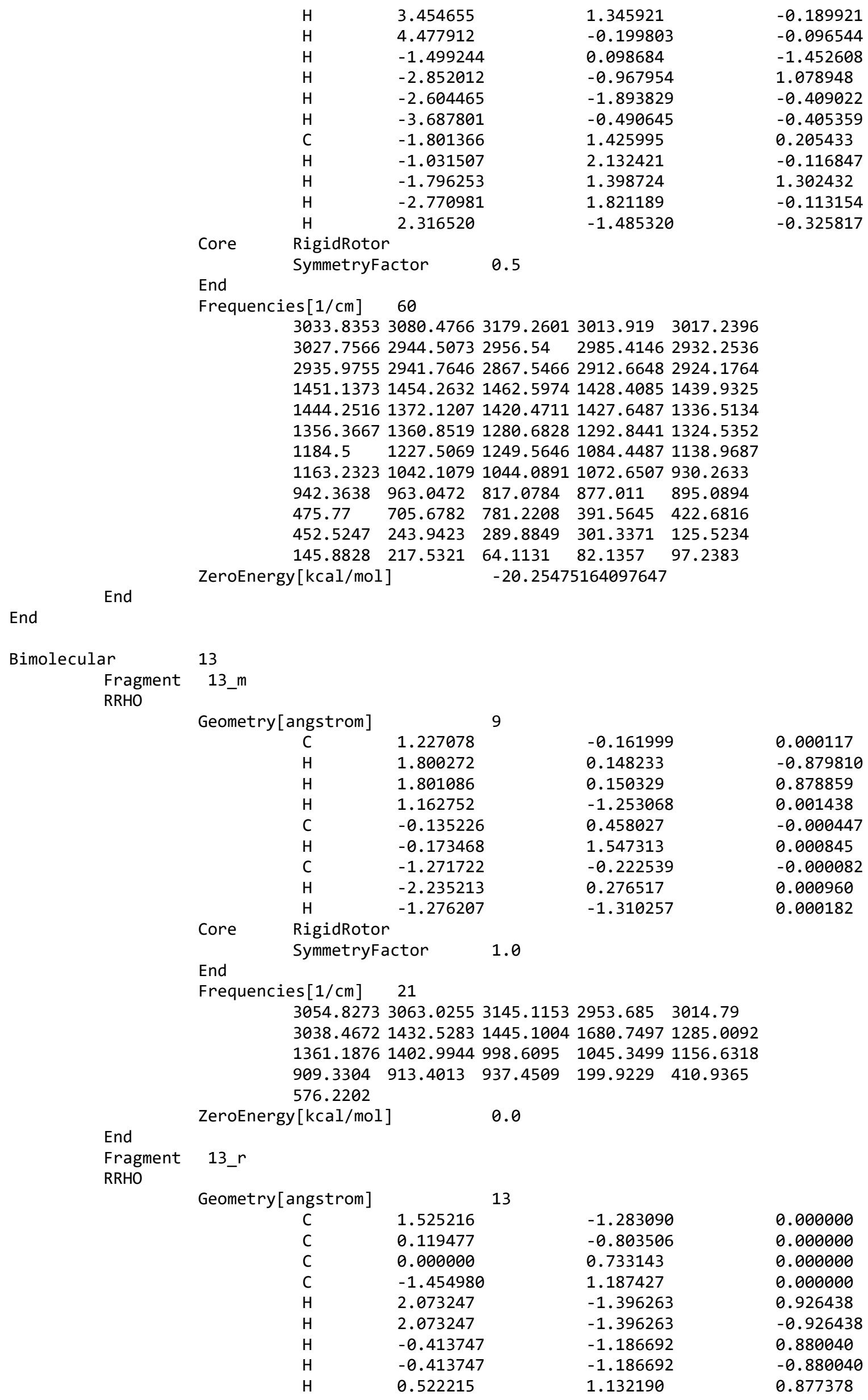




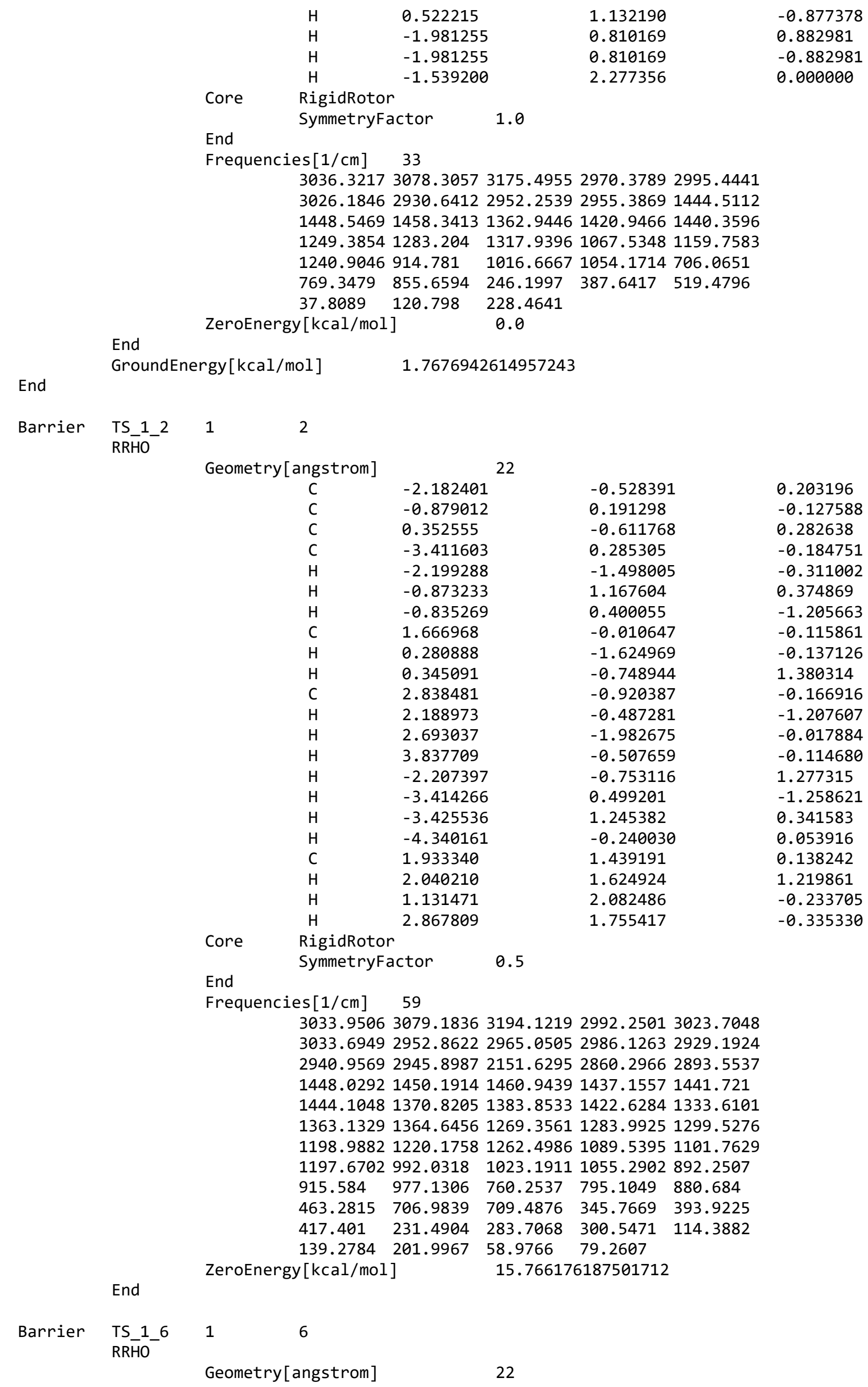




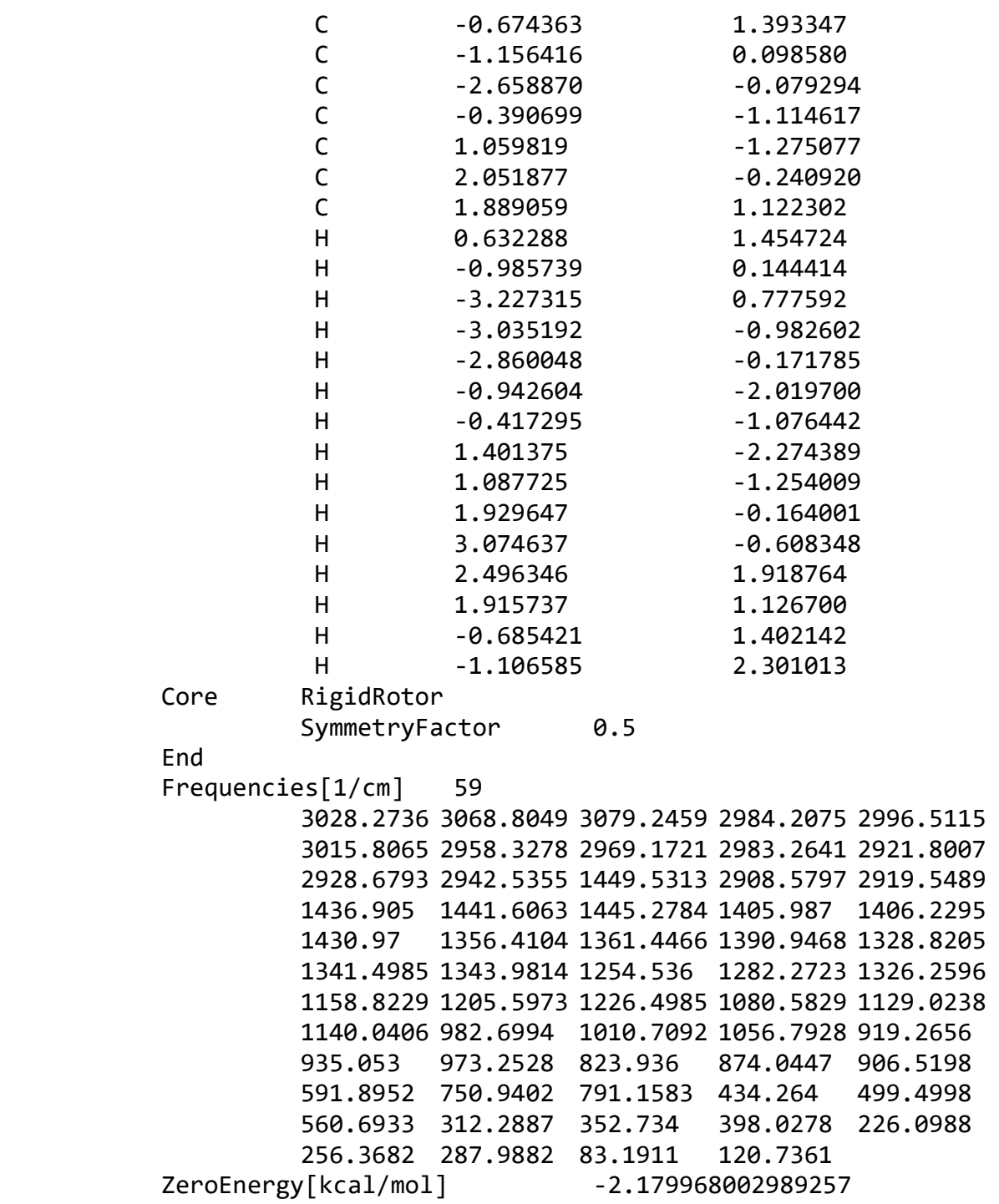

Barrier

1

13

RRHO

Geometry[angstrom]

Core

\begin{tabular}{|c|c|c|c|}
\hline try & & & \\
\hline $\mathrm{C}$ & 3.011392 & -0.736245 & -0.064122 \\
\hline C & 2.022041 & 0.295539 & 0.412640 \\
\hline C & 0.180756 & -0.866641 & -0.088657 \\
\hline C & -0.962531 & 0.072189 & 0.090566 \\
\hline C & -2.333752 & -0.579140 & -0.139832 \\
\hline C & -3.473972 & 0.416086 & 0.047186 \\
\hline C & 1.884612 & 1.494383 & -0.204502 \\
\hline $\mathrm{H}$ & 3.983516 & -0.584105 & 0.417291 \\
\hline $\mathrm{H}$ & 2.687369 & -1.755860 & 0.161088 \\
\hline $\mathrm{H}$ & 3.158819 & -0.655335 & -1.145363 \\
\hline $\mathrm{H}$ & 1.709985 & 0.220763 & 1.454508 \\
\hline $\mathrm{H}$ & 1.299908 & 2.295932 & 0.233549 \\
\hline $\mathrm{H}$ & 2.305842 & 1.666416 & -1.191327 \\
\hline $\mathrm{H}$ & 0.465225 & -1.143099 & -1.100416 \\
\hline $\mathrm{H}$ & 0.322680 & -1.648530 & 0.653917 \\
\hline $\mathrm{H}$ & -0.849895 & 0.920211 & -0.598051 \\
\hline $\mathrm{H}$ & -0.941431 & 0.496126 & 1.105369 \\
\hline $\mathrm{H}$ & -2.364613 & -1.002126 & -1.151158 \\
\hline $\mathrm{H}$ & -2.454037 & -1.423216 & 0.549969 \\
\hline $\mathrm{H}$ & -3.378280 & 1.253196 & -0.652016 \\
\hline $\mathrm{H}$ & -3.466517 & 0.830990 & 1.060484 \\
\hline $\mathrm{H}$ & -4.449856 & -0.048396 & -0.117514 \\
\hline RigidRoto & & & \\
\hline
\end{tabular}

0.306765

$-0.305323$

$-0.063534$

0.241375

$-0.226303$

0.317359

$-0.307403$

$-0.010343$

$-1.392365$

$-0.436811$

$-0.555754$

1.010082

$-0.043253$

1.341604

0.067332

$-1.325541$

1.407018

0.153853

0.122763

$-1.399168$

1.400101

$-0.117139$ 
End

SymmetryFactor $\quad 0.5$

Frequencies $[1 / \mathrm{cm}] \quad 59$

3059.32453123 .22753150 .04563032 .95313034 .1098 3047.10333018 .63683023 .53093029 .69852952 .9595

2962.79382988 .25562917 .65572947 .11452952 .0536

1451.34361458 .44641542 .13161436 .09151443 .8032

1446.32211390 .81381420 .93971434 .48861328 .9358

$1357.457 \quad 1364.29851248 .16231257 .61681284 .7043$

1146.48151168 .33541242 .34071022 .21251062 .4697

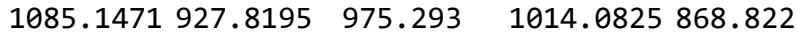

$\begin{array}{lllll}895.8943 & 907.8554 & 777.5329 & 781.794 & 837.3792\end{array}$

$\begin{array}{llllll}540.8591 & 546.3868 & 707.3431 & 305.322 & 400.7278\end{array}$

$\begin{array}{lllll}410.9918 & 216.1383 & 235.9629 & 247.9283 & 91.2369\end{array}$

$\begin{array}{llll}124.822 & 163.8785 & 46.7074 & 58.4824\end{array}$

End

ZeroEnergy $[\mathrm{kcal} / \mathrm{mol}] \quad 9.603405388009186$

End 


\section{Input file for $M E S S$ for case 5}

TemperatureList [K]

PressureList[torr]

1E6 1E9

EnergyStepoverTemperature

ExcessEnergyOverTemperature

ModelEnergyLimit [ $\mathrm{kcal} / \mathrm{mol}]$

CalculationMethod

ChemicalEigenvalueMax

ChemicalEigenvalueMin

EigenvalueOutput

Model

EnergyRelaxation

Exponential

Factor $[1 / \mathrm{cm}]$

Power

End

CollisionFrequency

LennardJones

Epsilons [1/cm]

Sigmas [angstrom]

End

Masses [amu ]

$\begin{array}{ll}\text { Well } & 1 \\ & \text { Species } \\ & \text { RRHO }\end{array}$

400.

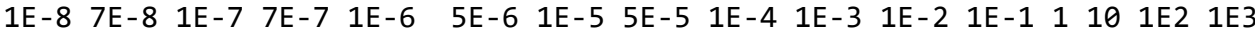

.2

30

400

direct

.2

$1 \mathrm{E}-7$

eval.out

130.

0

$124.342 \quad 197.807$

3.6557 .61

$4099 ! \mathrm{Ar}$

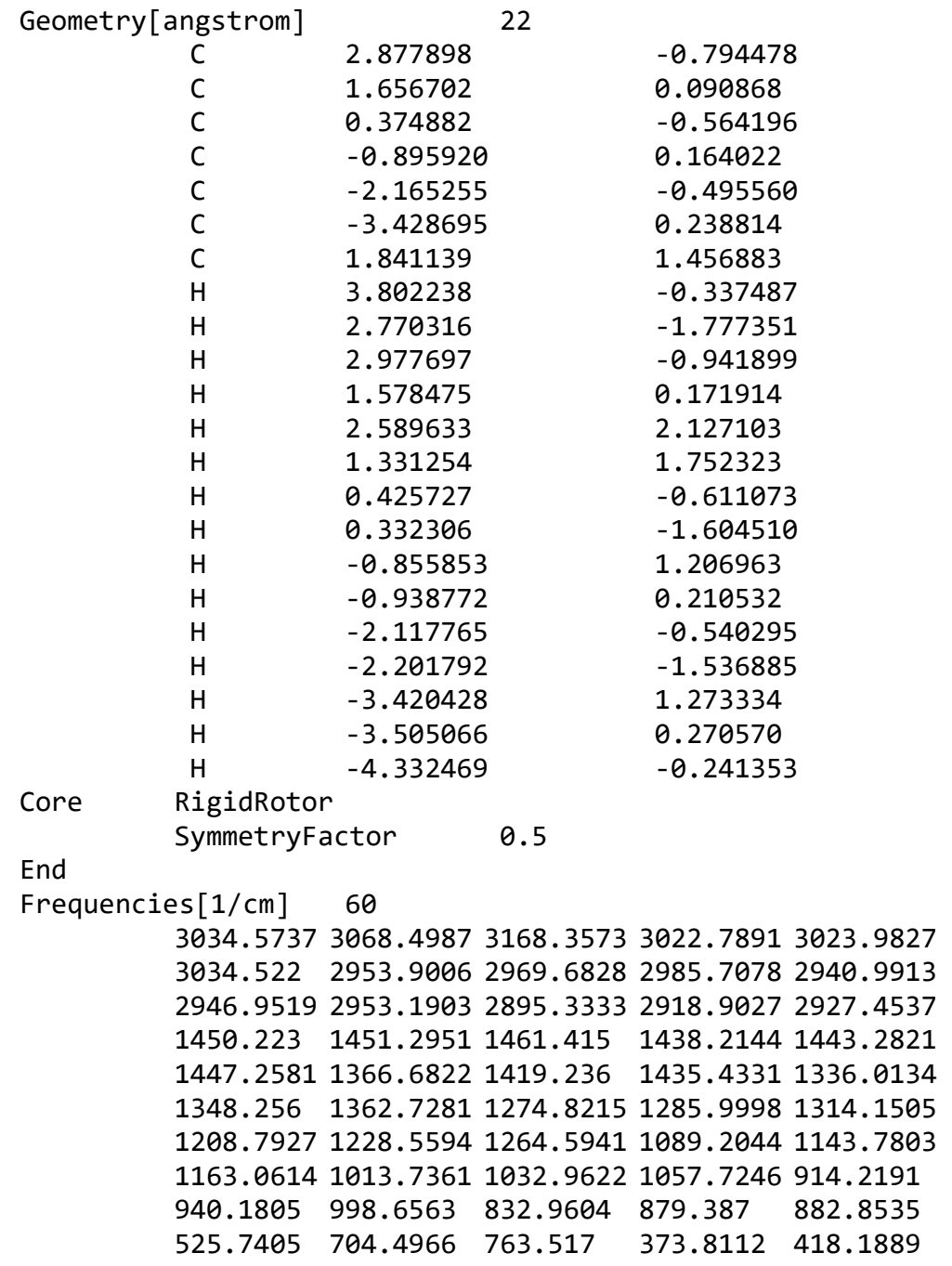

0.027662

0.333186

$-0.186344$

0.235953

$-0.292788$

0.141314

$-0.232058$

0.391760

0.498471

$-1.052897$

1.429911

0.172026

$-1.142041$

$-1.284847$

0.163433

$-0.107692$

1.333670

$-1.388538$

0.052687

$-0.217358$

1.233171

$-0.243309$ 
End

$\begin{array}{lllll}450.8962 & 228.1196 & 282.6025 & 285.4435 & 120.0641\end{array}$

$\begin{array}{lllll}154.2477 & 223.7787 & 55.6091 & 75.2174 & 92.5654\end{array}$

ZeroEnergy [kcal/mol] $\quad-19.92656417248466$

End

Well 2

Species

RRHO

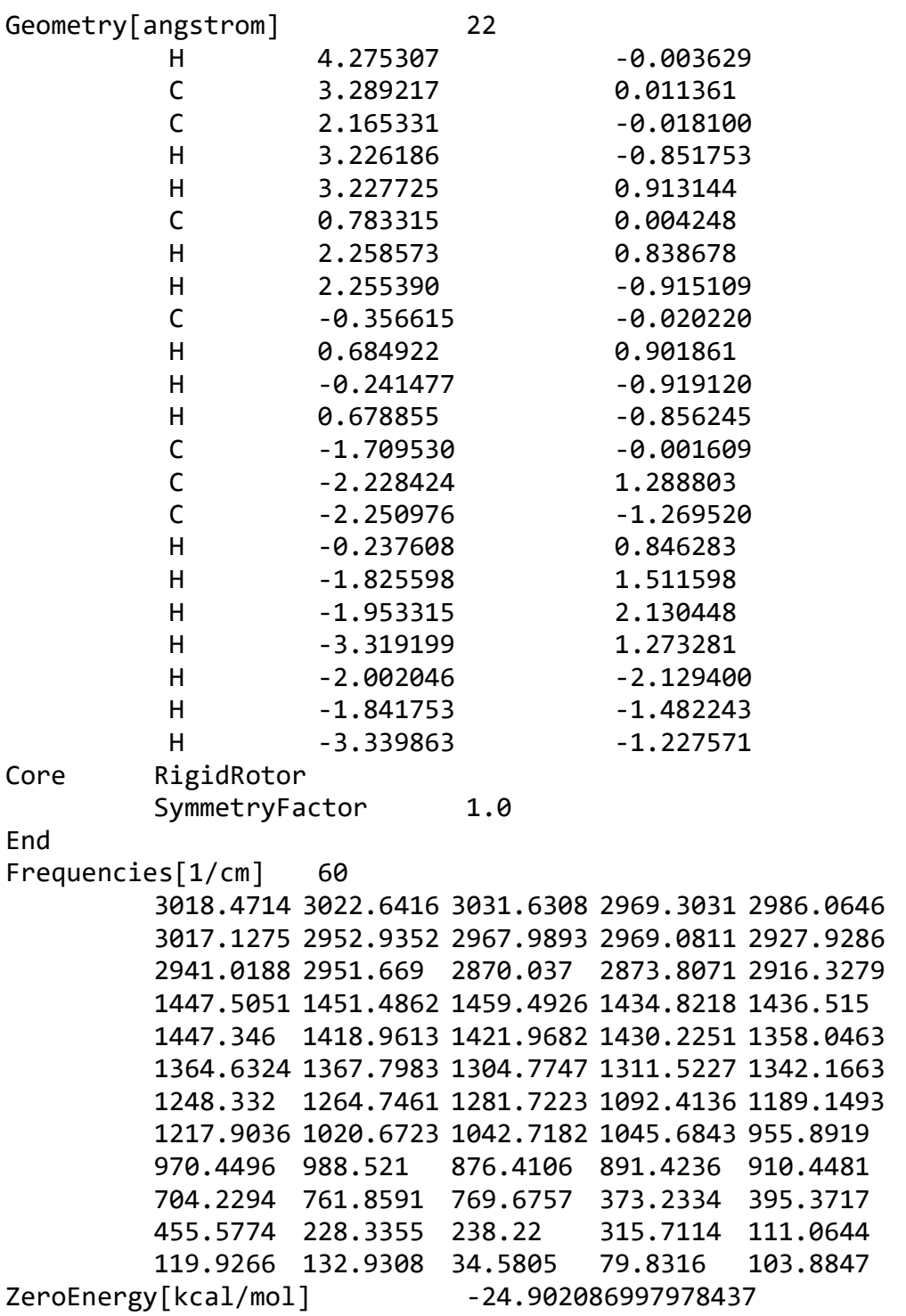

End

End .902086997978437

Well 6

Species RRHO

$\begin{array}{cl}\text { Geometry[angstrom] } & \\ \text { C } & -1.570543 \\ \text { C } & -0.263497 \\ \text { C } & 0.987746 \\ \text { C } & -2.746698 \\ \text { H } & -0.163361 \\ \text { H } & -0.332205 \\ \text { C } & 2.274107 \\ \text { H } & 0.970629 \\ \text { H } & 1.000006 \\ \text { C } & 3.502665 \\ \text { H } & 2.223621\end{array}$

22

$\begin{array}{ll}0.024556 & -0.357283 \\ -0.583827 & 0.156491 \\ 0.214357 & -0.192748 \\ -0.884701 & -0.009564 \\ -1.601148 & -0.247578 \\ -0.696195 & 1.249624 \\ -0.515603 & 0.191244 \\ 1.187031 & 0.315006 \\ 0.428821 & -1.270347 \\ 0.268361 & -0.090837 \\ -0.772729 & 1.264770\end{array}$

$-0.550075$

0.479556

$-1.220911$

$-1.168118$

$-0.163744$

1.159347

1.105759

0.870971

$-0.789977$

1.492884

$-0.839185$

0.241551

$-0.291512$

$-0.322016$

1.536912

$-1.295319$

0.353123

$-0.393225$

0.309023

$-1.325478$

$-0.435225$ 


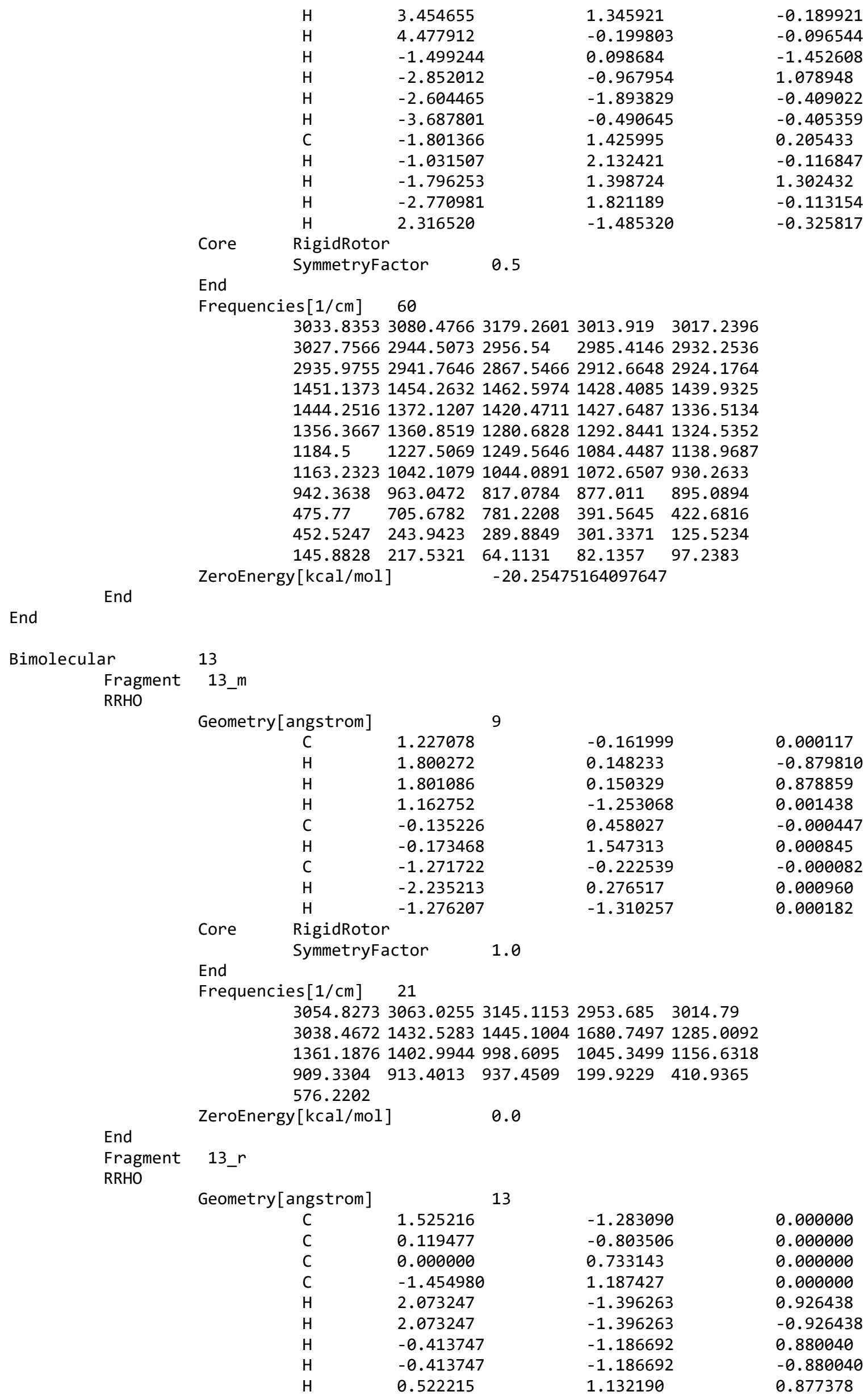




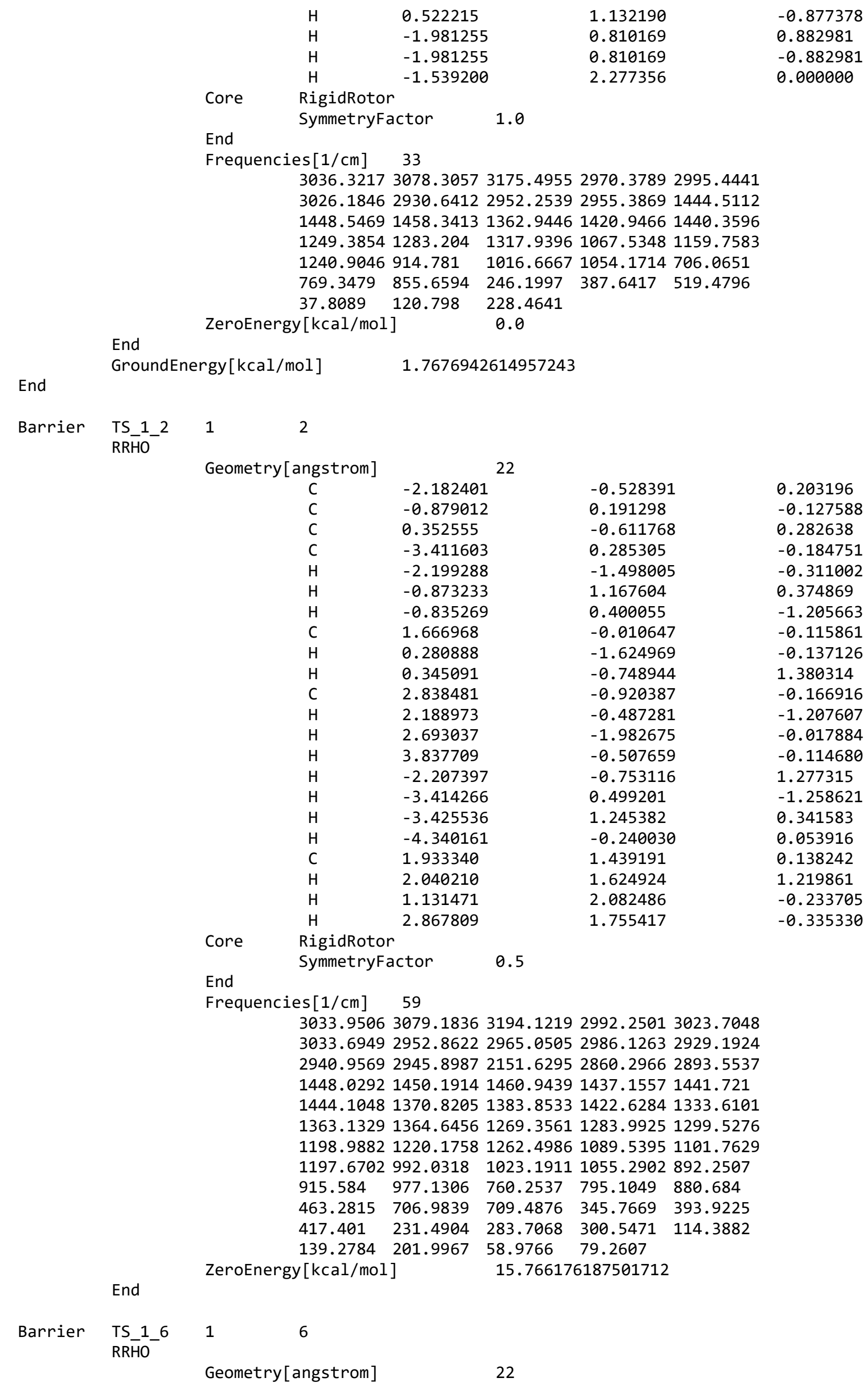


Core

$$
\begin{aligned}
& -0.674363 \\
& -1.156416 \\
& -2.658870 \\
& -0.390699 \\
& 1.059819 \\
& 2.051877 \\
& 1.889059 \\
& 0.632288 \\
& -0.985739 \\
& -3.227315 \\
& -3.035192 \\
& -2.860048 \\
& -0.942604 \\
& -0.417295 \\
& 1.401375 \\
& 1.087725 \\
& 1.929647 \\
& 3.074637 \\
& 2.496346
\end{aligned}
$$

1.393347

0.306765

0.098580

$-0.305323$

$\begin{array}{ll}C & -1.156416 \\ \text { C } & -2.658870\end{array}$

$-0.079294$

$-0.063534$

$-1.114617$

0.241375

$-1.275077$

$-0.226303$

$-0.240920$

0.317359

$-0.307403$

1.122302

$-0.010343$

1.454724

-0.010343
-1.392365

0.777592

$-0.436811$

$-0.982602$

$-0.555754$

$-0.171785$

$-2.019700$

1.010082

$-1.076442$

$-0.043253$

1.341604

$-2.274389$

0.067332

$-1.254009$

$-1.325541$

$-0.164001$

1.407018

0.153853

1.918764

0.122763

1.126700

$-1.399168$

1.402142

1.400101

H $\quad-1.106585$

2. 301013

$-0.117139$

End

SymmetryFactor

0.5

Frequencies [1/cm] 59

3028.27363068 .80493079 .24592984 .20752996 .5115

3015.80652958 .32782969 .17212983 .26412921 .8007

2928.67932942 .53551449 .53132908 .57972919 .5489

$\begin{array}{lllll}1436.905 & 1441.6063 & 1445.2784 & 1405.987 & 1406.2295\end{array}$

$1430.97 \quad 1356.41041361 .44661390 .94681328 .8205$

$1341.49851343 .98141254 .536 \quad 1282.27231326 .2596$

1158.82291205 .59731226 .49851080 .58291129 .0238

$1140.0406982 .6994 \quad 1010.70921056 .7928919 .2656$

$\begin{array}{lllll}935.053 & 973.2528 & 823.936 & 874.0447 & 906.5198\end{array}$

$\begin{array}{llllll}591.8952 & 750.9402 & 791.1583 & 434.264 & 499.4998\end{array}$

$\begin{array}{lllll}560.6933 & 312.2887 & 352.734 & 398.0278 & 226.0988\end{array}$

$\begin{array}{llll}256.3682 & 287.9882 & 83.1911 & 120.7361\end{array}$

End

Barrier

$\begin{array}{ll}\text { TS_1_13 } & 1 \\ \text { RRHO } & \end{array}$

13

Geometry[angstrom]

C 3.011392

22

C $\quad 2.022041$

0.180756

$-0.962531$

$-2.333752$

$-3.473972$

1.884612

3.983516

2.687369

3.158819

1.709985

1.299908

2.305842

0.465225

0.322680

$-0.849895$

$-0.941431$

$-2.364613$

$-2.454037$

$-3.378280$

$-3.466517$

$-4.449856$

$-0.736245$

$-0.064122$

0.295539

$-0.866641$

0.072189

0.412640

$-0.088657$

0.090566

$-0.139832$

$-0.579140$

0.047186

$-0.204502$

1.494383

0.417291

0.161088

$-1.755860$

$-0.655335$

$-1.145363$

0.220763

1.454508

2.295932

0.233549

1.666416

$-1.191327$

$-1.143099$

$-1.100416$

$-1.648530$

0.653917

$-0.598051$

0.496126

1.105369

$-1.002126$

$-1.151158$

0.549969

1.253196

$-0.652016$

0.830990

1.060484

Core RigidRotor

$-0.048396$ 


\begin{tabular}{|c|c|c|c|c|c|c|c|}
\hline & & SymmetryF & actor & 0.5 & & & \\
\hline & & End & & & & & \\
\hline & & Frequencies $[1 / \mathrm{cm}]$ & 59 & & & & \\
\hline & & 3059.3245 & 3123.2275 & 3150.0456 & 3032.9531 & 3034.1098 & \\
\hline & & 3047.1033 & 3018.6368 & 3023.5309 & 3029.6985 & 2952.9595 & \\
\hline & & 2962.7938 & 2988.2556 & 2917.6557 & 2947.1145 & 2952.0536 & \\
\hline & & 1451.3436 & 1458.4464 & 1542.1316 & 1436.0915 & 1443.8032 & \\
\hline & & 1446.3221 & 1390.8138 & 1420.9397 & 1434.4886 & 1328.9358 & \\
\hline & & 1357.457 & 1364.2985 & 1248.1623 & 1257.6168 & 1284.7043 & \\
\hline & & 1146.4815 & 1168.3354 & 1242.3407 & 1022.2125 & 1062.4697 & \\
\hline & & 1085.1471 & 927.8195 & 975.293 & 1014.0825 & 868.822 & \\
\hline & & 895.8943 & 907.8554 & 777.5329 & 781.794 & 837.3792 & \\
\hline & & 540.8591 & 546.3868 & 707.3431 & 305.322 & 400.7278 & \\
\hline & & 410.9918 & 216.1383 & 235.9629 & 247.9283 & 91.2369 & \\
\hline & & 124.822 & 163.8785 & 46.7074 & 58.4824 & & \\
\hline & & ZeroEnergy $[\mathrm{kcal} / \mathrm{mo}]$ & & 9.603405 & 388009186 & & \\
\hline & End & & & & & & \\
\hline Barrier & TS_2_6 & 2 & & & & & \\
\hline & & Geometry[angstrom] & & 22 & & & \\
\hline & & $C$ & 1.288281 & & 1.435281 & & 0.199423 \\
\hline & & C & 2.039331 & & 0.218377 & & -0.282710 \\
\hline & & C & 1.326579 & & -1.06958 & & 0.149687 \\
\hline & & $\mathrm{H}$ & 3.072474 & & 0.214525 & & 0.091540 \\
\hline & & $\mathrm{H}$ & 2.107306 & & 0.232900 & & -1.378303 \\
\hline & & C & -0.081081 & & -1.13145 & & -0.453897 \\
\hline & & $\mathrm{H}$ & 1.905829 & & -1.94640 & & -0.157136 \\
\hline & & $\mathrm{H}$ & 1.267292 & & -1.10116 & & 1.246047 \\
\hline & & $\mathrm{H}$ & 1.324313 & & 1.603929 & & 1.276929 \\
\hline & & $\mathrm{H}$ & 1.402563 & & 2.352468 & & -0.374979 \\
\hline & & $\mathrm{H}$ & 0.009917 & & -1.14049 & & -1.548919 \\
\hline & & $\mathrm{H}$ & -0.574703 & & -2.07519 & & -0.173060 \\
\hline & & $\mathrm{C}$ & -0.939697 & & 0.048113 & & -0.030602 \\
\hline & & C & $-1.45601 €$ & & -0.04806 & & 1.386664 \\
\hline & & $\mathrm{C}$ & -1.99172 & & 0.454676 & & -1.032888 \\
\hline & & $\mathrm{H}$ & -0.016735 & & 0.990254 & & 0.019539 \\
\hline & & $\mathrm{H}$ & -0.650836 & & -0.25261 & & 2.099890 \\
\hline & & $\mathrm{H}$ & $-2.19191 \varepsilon$ & & -0.86058 & & 1.476608 \\
\hline & & $\mathrm{H}$ & -1.949331 & & 0.879497 & & 1.694778 \\
\hline & & $\mathrm{H}$ & -2.73999 & & -0.34297 & & -1.156740 \\
\hline & & $\mathrm{H}$ & -1.554301 & & 0.646963 & & -2.017891 \\
\hline & & $\mathrm{H}$ & -2.525952 & & 1.354846 & & -0.712366 \\
\hline & & RigidRoto & & & & & \\
\hline & & Symmetryf & actor & 1.0 & & & \\
\hline & & End & & & & & \\
\hline & & Frequencies $[1 / \mathrm{cm}]$ & 59 & & & & \\
\hline & & 3023.7321 & 3024.4511 & 3093.9621 & 2993.1499 & 3002.0259 & \\
\hline & & 3007.708 & 2959.8398 & 2970.8857 & 2987.4088 & 2919.1728 & \\
\hline & & 2923.9982 & 2942.219 & 1475.9129 & 2903.3092 & 2911.6245 & \\
\hline & & 1441.3605 & 1444.2346 & 1451.5444 & 1428.9137 & 1431.9824 & \\
\hline & & 1439.8112 & 1370.3101 & 1407.8094 & 1422.7009 & 1325.7566 & \\
\hline & & 1345.7946 & 1365.9875 & 1250.4455 & 1315.2358 & 1321.4086 & \\
\hline & & 1184.7941 & 1213.4085 & 1221.6341 & 1065.7144 & 1108.4401 & \\
\hline & & 1170.8827 & 967.948 & 1025.8025 & 1039.7664 & 915.4744 & \\
\hline & & 925.6223 & 963.9256 & 819.2047 & 881.2232 & 899.3584 & \\
\hline & & 560.9214 & 751.4071 & 795.117 & 414.6789 & 453.3534 & \\
\hline & & 492.9938 & 277.1293 & 341.3522 & 362.3296 & 181.461 & \\
\hline & & 194.7343 & 239.3295 & 96.3736 & 127.9081 & & \\
\hline & & ZeroEnergy $[\mathrm{kcal} / \mathrm{mo}]$ & & -1.52647 & 6166493875 & & \\
\hline
\end{tabular}

End 


\section{Input file for MESS for case 6}

TemperatureList [K]

PressureList[torr]

1E6 1E9 1E10

EnergyStepOverTemperature

ExcessEnergyOverTemperature

ModelEnergyLimit [ $\mathrm{kcal} / \mathrm{mol}]$

CalculationMethod

ChemicalEigenvalueMax

ChemicalEigenvalueMin

EigenvalueOutput

Model

EnergyRelaxation

Exponential

Factor $[1 / \mathrm{cm}]$

Power

End

CollisionFrequency

LennardJones

Epsilons [1/cm]

Sigmas [angstrom]

End

Masses [amu ]

$\begin{array}{ll}\text { Well } & 1 \\ & \text { Species } \\ & \text { RRHO }\end{array}$

400.

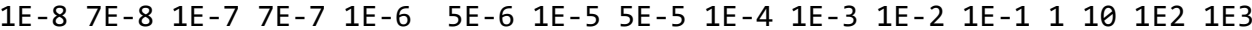

.2

30

400

direct

.2

$1 \mathrm{E}-7$

eval.out

130.

0

$124.342 \quad 197.807$

3.6557 .61

$4099 ! \mathrm{Ar}$

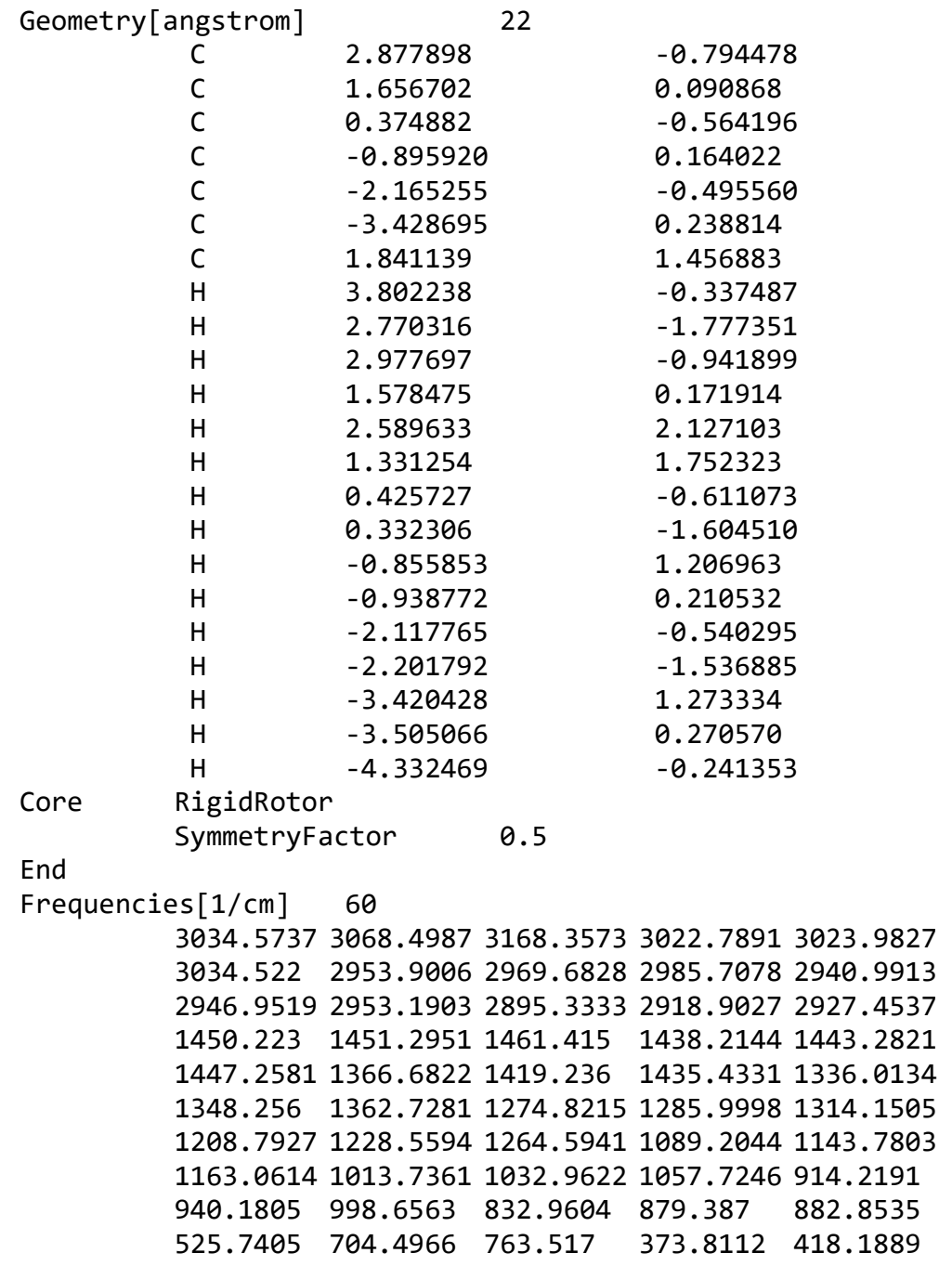

0.027662

0.333186

$-0.186344$

0.235953

$-0.292788$

0.141314

$-0.232058$

0.391760

0.498471

$-1.052897$

1.429911

0.172026

$-1.142041$

$-1.284847$

0.163433

$-0.107692$

1.333670

$-1.388538$

0.052687

$-0.217358$

1.233171

$-0.243309$ 
End

$\begin{array}{lllll}450.8962 & 228.1196 & 282.6025 & 285.4435 & 120.0641\end{array}$

$\begin{array}{lllll}154.2477 & 223.7787 & 55.6091 & 75.2174 & 92.5654\end{array}$

ZeroEnergy[kcal/mol] $\quad-19.92656417248466$

End

Well 2

Species

RRHO

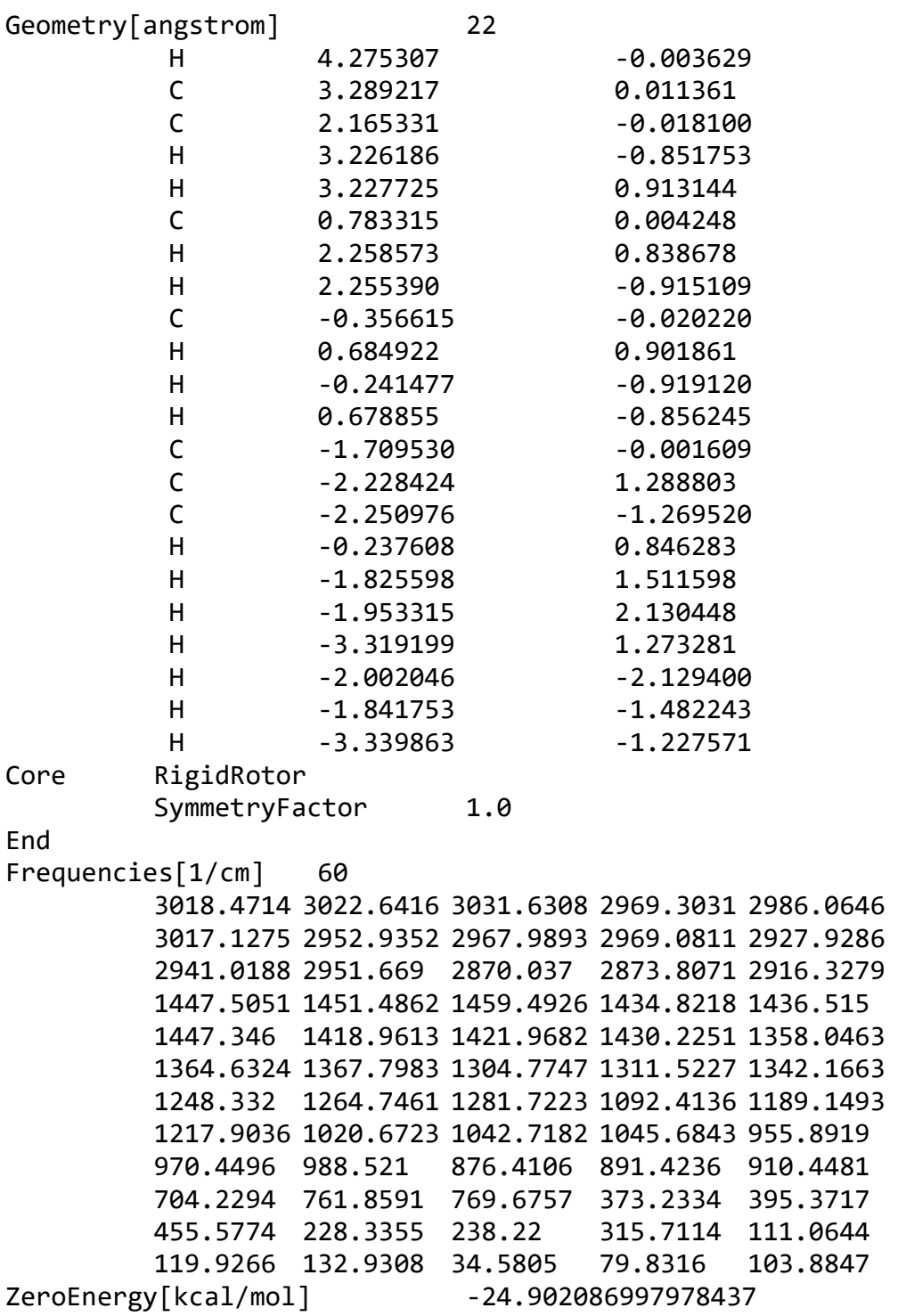

End

End .902086997978437

Well 6

Species RRHO

$\begin{array}{cl}\text { Geometry[angstrom] } & \\ \text { C } & -1.570543 \\ \text { C } & -0.263497 \\ \text { C } & 0.987746 \\ \text { C } & -2.746698 \\ \text { H } & -0.163361 \\ \text { H } & -0.332205 \\ \text { C } & 2.274107 \\ \text { H } & 0.970629 \\ \text { H } & 1.000006 \\ \text { C } & 3.502665 \\ \text { H } & 2.223621\end{array}$

22

$\begin{array}{ll}0.024556 & -0.357283 \\ -0.583827 & 0.156491 \\ 0.214357 & -0.192748 \\ -0.884701 & -0.009564 \\ -1.601148 & -0.247578 \\ -0.696195 & 1.249624 \\ -0.515603 & 0.191244 \\ 1.187031 & 0.315006 \\ 0.428821 & -1.270347 \\ 0.268361 & -0.090837 \\ -0.772729 & 1.264770\end{array}$

$-0.077987$

$-0.550075$

0.479556

$-1.220911$

$-1.168118$

$-0.163744$

1.159347

1.105759

0.870971

$-0.789977$

1.492884

$-0.839185$

0.241551

$-0.291512$

$-0.322016$

1.536912

$-1.295319$

0.353123

$-0.393225$

0.309023

$-1.325478$

$-0.435225$ 


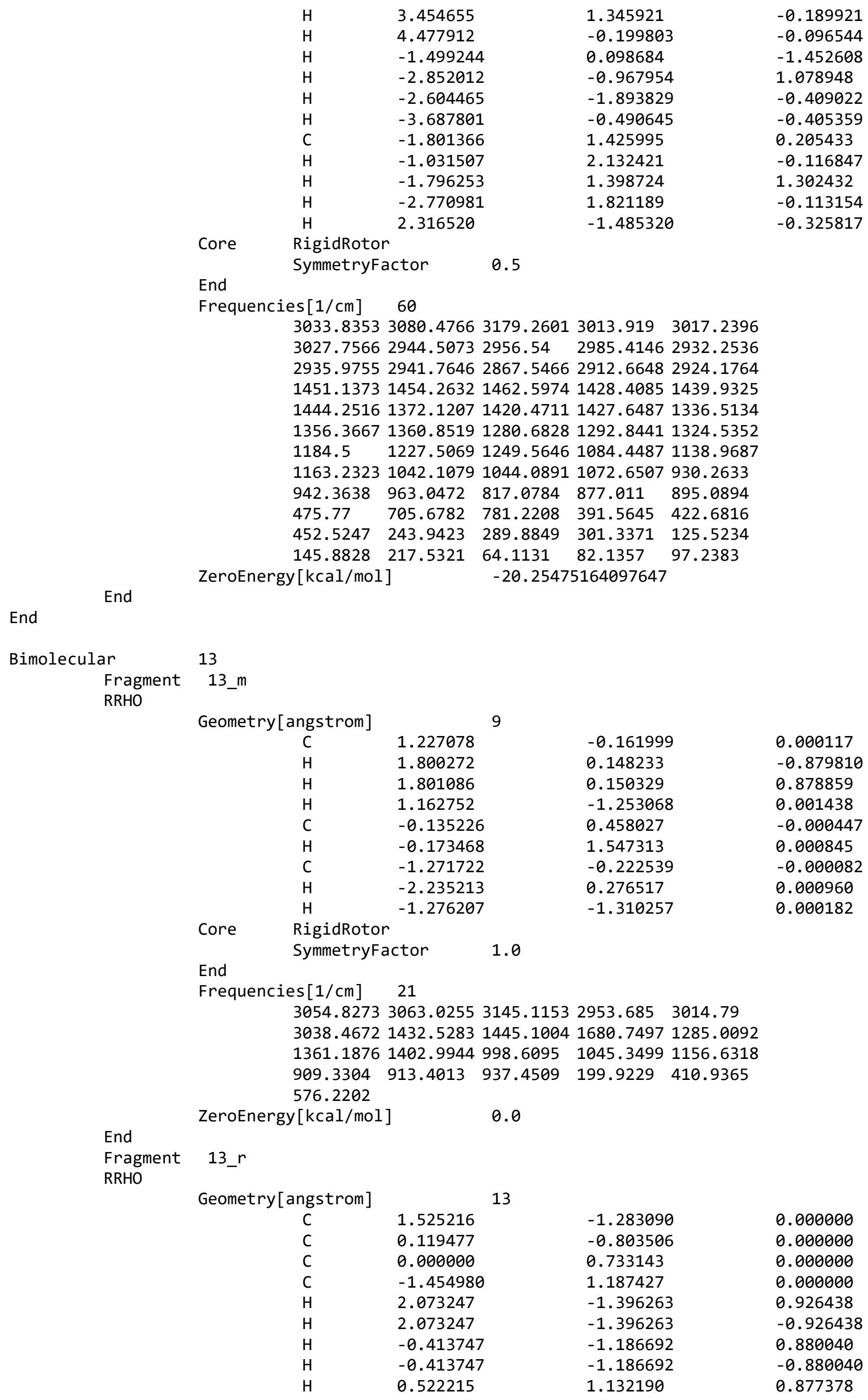




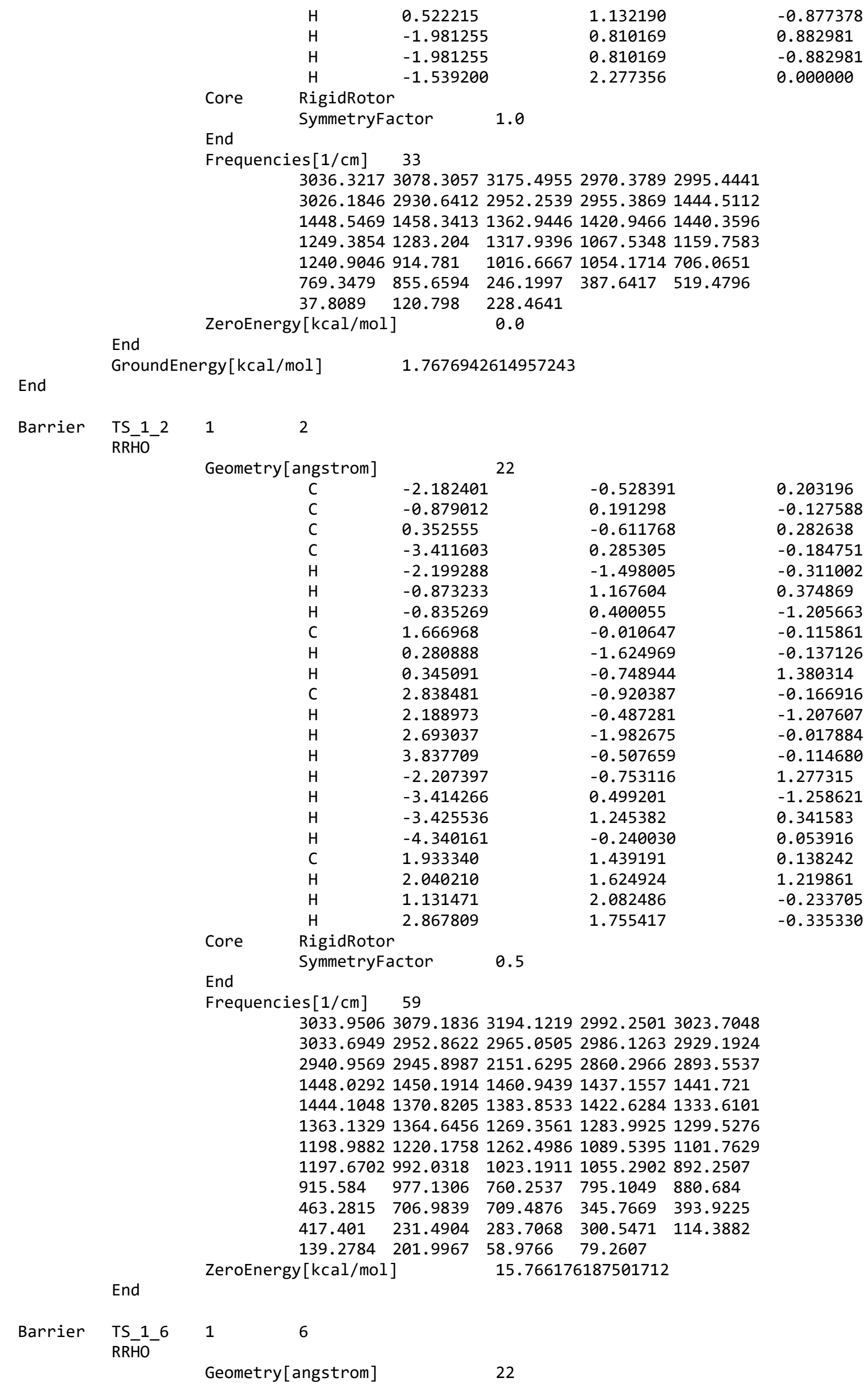




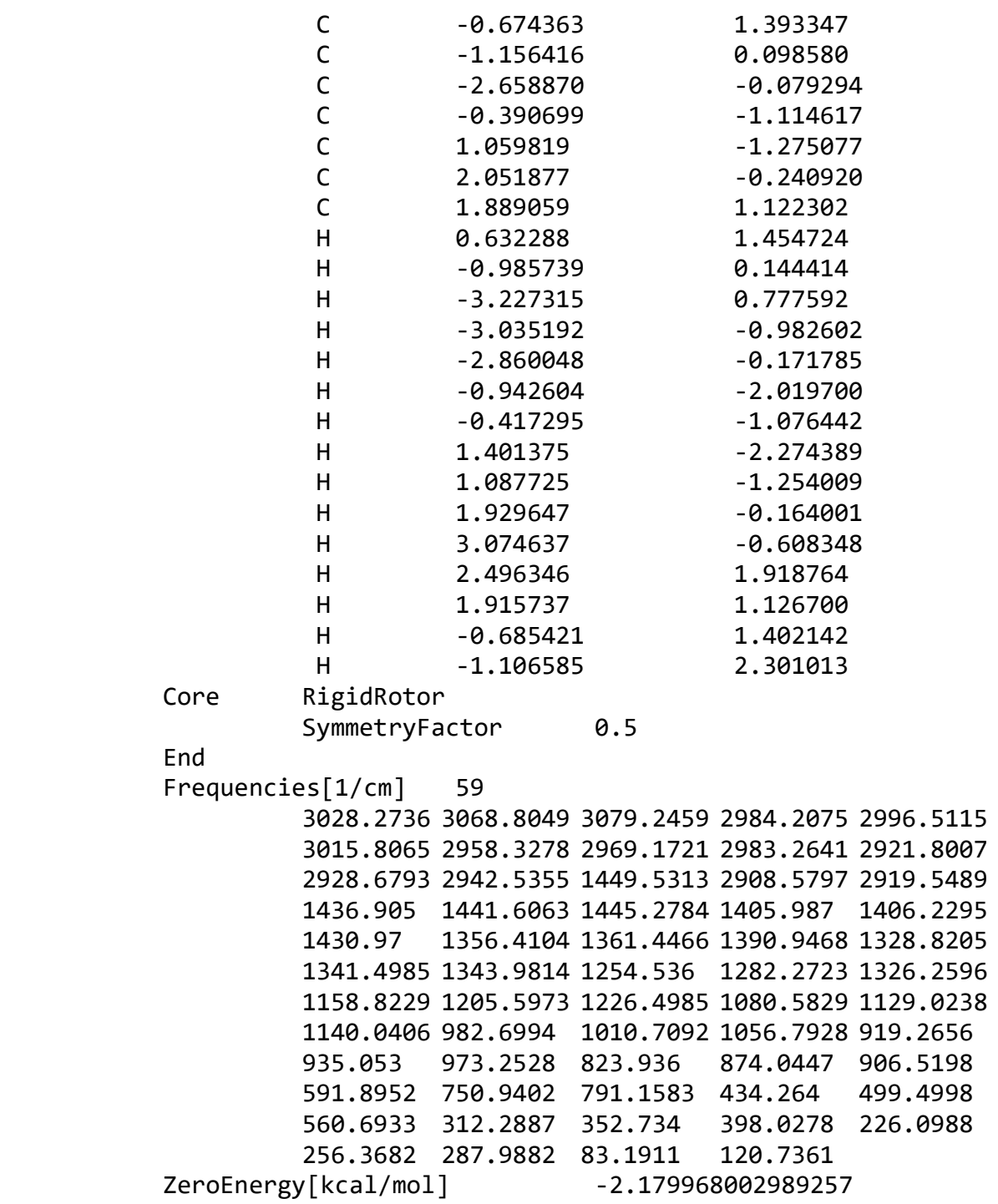

Barrier

1

13

RRHO

Geometry [angstrom]

$\begin{array}{clll}\text { C } & 3.011392 & -0.736245 & -0.064122 \\ \text { C } & 2.022041 & 0.295539 & 0.412640 \\ \text { C } & 0.180756 & -0.866641 & -0.088657 \\ \text { C } & -0.962531 & 0.072189 & 0.090566 \\ \text { C } & -2.333752 & -0.579140 & -0.139832 \\ \text { C } & -3.473972 & 0.416086 & 0.047186 \\ \text { C } & 1.884612 & 1.494383 & -0.204502 \\ \text { H } & 3.983516 & -0.584105 & 0.417291 \\ \text { H } & 2.687369 & -1.755860 & 0.161088 \\ \text { H } & 3.158819 & -0.655335 & -1.145363 \\ \text { H } & 1.709985 & 0.220763 & 1.454508 \\ \text { H } & 1.299908 & 2.295932 & 0.233549 \\ \text { H } & 2.305842 & 1.666416 & -1.191327 \\ \text { H } & 0.465225 & -1.143099 & -1.100416 \\ \text { H } & 0.322680 & -1.648530 & 0.653917 \\ \text { H } & -0.849895 & 0.920211 & -0.598051 \\ \text { H } & -0.941431 & 0.496126 & 1.105369 \\ \text { H } & -2.364613 & -1.002126 & -1.151158 \\ \text { H } & -2.454037 & -1.423216 & 0.549969 \\ \text { H } & -3.378280 & 1.253196 & -0.652016 \\ \text { H } & -3.466517 & 0.830990 & 1.060484 \\ \text { H } & -4.449856 & -0.048396 & -0.117514 \\ \text { RigidRotor } & & & \end{array}$

0.306765

$-0.305323$

$-0.063534$

0.241375

$-0.226303$

0.317359

$-0.307403$

$-0.010343$

$-1.392365$

$-0.436811$

$-0.555754$

1.010082

$-0.043253$

1.341604

0.067332

$-1.325541$

1.407018

0.153853

0.122763

$-1.399168$

1.400101

$-0.117139$ 


\begin{tabular}{|c|c|c|c|c|c|c|c|}
\hline & & SymmetryF & actor & 0.5 & & & \\
\hline & & End & & & & & \\
\hline & & Frequencies $[1 / \mathrm{cm}]$ & 59 & & & & \\
\hline & & 3059.3245 & 3123.2275 & 3150.0456 & 3032.9531 & 3034.1098 & \\
\hline & & 3047.1033 & 3018.6368 & 3023.5309 & 3029.6985 & 2952.9595 & \\
\hline & & 2962.7938 & 2988.2556 & 2917.6557 & 2947.1145 & 2952.0536 & \\
\hline & & 1451.3436 & 1458.4464 & 1542.1316 & 1436.0915 & 1443.8032 & \\
\hline & & 1446.3221 & 1390.8138 & 1420.9397 & 1434.4886 & 1328.9358 & \\
\hline & & 1357.457 & 1364.2985 & 1248.1623 & 1257.6168 & 1284.7043 & \\
\hline & & 1146.4815 & 1168.3354 & 1242.3407 & 1022.2125 & 1062.4697 & \\
\hline & & 1085.1471 & 927.8195 & 975.293 & 1014.0825 & 868.822 & \\
\hline & & 895.8943 & 907.8554 & 777.5329 & 781.794 & 837.3792 & \\
\hline & & 540.8591 & 546.3868 & 707.3431 & 305.322 & 400.7278 & \\
\hline & & 410.9918 & 216.1383 & 235.9629 & 247.9283 & 91.2369 & \\
\hline & & 124.822 & 163.8785 & 46.7074 & 58.4824 & & \\
\hline & & ZeroEnergy $[\mathrm{kcal} / \mathrm{mo}]$ & & 9.603405 & 388009186 & & \\
\hline & End & & & & & & \\
\hline Barrier & TS_2_6 & 2 & & & & & \\
\hline & & Geometry[angstrom] & & 22 & & & \\
\hline & & $C$ & 1.288281 & & 1.435281 & & 0.199423 \\
\hline & & C & 2.039331 & & 0.218377 & & -0.282710 \\
\hline & & C & 1.326579 & & -1.06958 & & 0.149687 \\
\hline & & $\mathrm{H}$ & 3.072474 & & 0.214525 & & 0.091540 \\
\hline & & $\mathrm{H}$ & 2.107306 & & 0.232900 & & -1.378303 \\
\hline & & C & -0.081081 & & -1.13145 & & -0.453897 \\
\hline & & $\mathrm{H}$ & 1.905829 & & -1.94640 & & -0.157136 \\
\hline & & $\mathrm{H}$ & 1.267292 & & -1.10116 & & 1.246047 \\
\hline & & $\mathrm{H}$ & 1.324313 & & 1.603929 & & 1.276929 \\
\hline & & $\mathrm{H}$ & 1.402563 & & 2.352468 & & -0.374979 \\
\hline & & $\mathrm{H}$ & 0.009917 & & -1.14049 & & -1.548919 \\
\hline & & $\mathrm{H}$ & -0.574703 & & -2.07519 & & -0.173060 \\
\hline & & $\mathrm{C}$ & -0.939697 & & 0.048113 & & -0.030602 \\
\hline & & C & $-1.45601 €$ & & -0.04806 & & 1.386664 \\
\hline & & $\mathrm{C}$ & -1.99172 & & 0.454676 & & -1.032888 \\
\hline & & $\mathrm{H}$ & -0.016735 & & 0.990254 & & 0.019539 \\
\hline & & $\mathrm{H}$ & -0.650836 & & -0.25261 & & 2.099890 \\
\hline & & $\mathrm{H}$ & $-2.19191 \varepsilon$ & & -0.86058 & & 1.476608 \\
\hline & & $\mathrm{H}$ & -1.949331 & & 0.879497 & & 1.694778 \\
\hline & & $\mathrm{H}$ & -2.73999 & & -0.34297 & & -1.156740 \\
\hline & & $\mathrm{H}$ & -1.554301 & & 0.646963 & & -2.017891 \\
\hline & & $\mathrm{H}$ & -2.525952 & & 1.354846 & & -0.712366 \\
\hline & & RigidRoto & & & & & \\
\hline & & Symmetryf & actor & 1.0 & & & \\
\hline & & End & & & & & \\
\hline & & Frequencies $[1 / \mathrm{cm}]$ & 59 & & & & \\
\hline & & 3023.7321 & 3024.4511 & 3093.9621 & 2993.1499 & 3002.0259 & \\
\hline & & 3007.708 & 2959.8398 & 2970.8857 & 2987.4088 & 2919.1728 & \\
\hline & & 2923.9982 & 2942.219 & 1475.9129 & 2903.3092 & 2911.6245 & \\
\hline & & 1441.3605 & 1444.2346 & 1451.5444 & 1428.9137 & 1431.9824 & \\
\hline & & 1439.8112 & 1370.3101 & 1407.8094 & 1422.7009 & 1325.7566 & \\
\hline & & 1345.7946 & 1365.9875 & 1250.4455 & 1315.2358 & 1321.4086 & \\
\hline & & 1184.7941 & 1213.4085 & 1221.6341 & 1065.7144 & 1108.4401 & \\
\hline & & 1170.8827 & 967.948 & 1025.8025 & 1039.7664 & 915.4744 & \\
\hline & & 925.6223 & 963.9256 & 819.2047 & 881.2232 & 899.3584 & \\
\hline & & 560.9214 & 751.4071 & 795.117 & 414.6789 & 453.3534 & \\
\hline & & 492.9938 & 277.1293 & 341.3522 & 362.3296 & 181.461 & \\
\hline & & 194.7343 & 239.3295 & 96.3736 & 127.9081 & & \\
\hline & & ZeroEnergy $[\mathrm{kcal} / \mathrm{mo}]$ & & -5.52647 & 6166493875 & & \\
\hline
\end{tabular}

End 
23. Input file for MESS for case 7

TemperatureList [K]

PressureList [torr]

2E3 6E3 1E4 1E6 1E9

EnergyStepOverTemperature

ExcessEnergyOverTemperature

ModelEnergyLimit [ $\mathrm{kcal} / \mathrm{mol}]$

CalculationMethod

ChemicalEigenvalueMax

ChemicalEigenvalueMin

EigenvalueOutput

Model

EnergyRelaxation

Exponential

Factor $[1 / \mathrm{cm}]$

Power

End

CollisionFrequency

LennardJones

Epsilons [1/cm]

Sigmas [angstrom]

End

Masses [amu ]

$\begin{array}{ll}\text { Well } & 1 \\ & \text { Species } \\ & \text { RRHO }\end{array}$

400. 500. 600. 700. 800.

1E-8 7E-8 1E-7 7E-7 1E-6 5E-6 1E-5 5E-5 1E-4 1E-3 1E-2 $1 \mathrm{E}-1 \quad 110$ 1E2 7E2

.2

30

400

direct

.2

$1 \mathrm{E}-7$

eval.out

130.

0

$124.342 \quad 197.807$

3.6557 .61

$4099 ! \mathrm{Ar}$

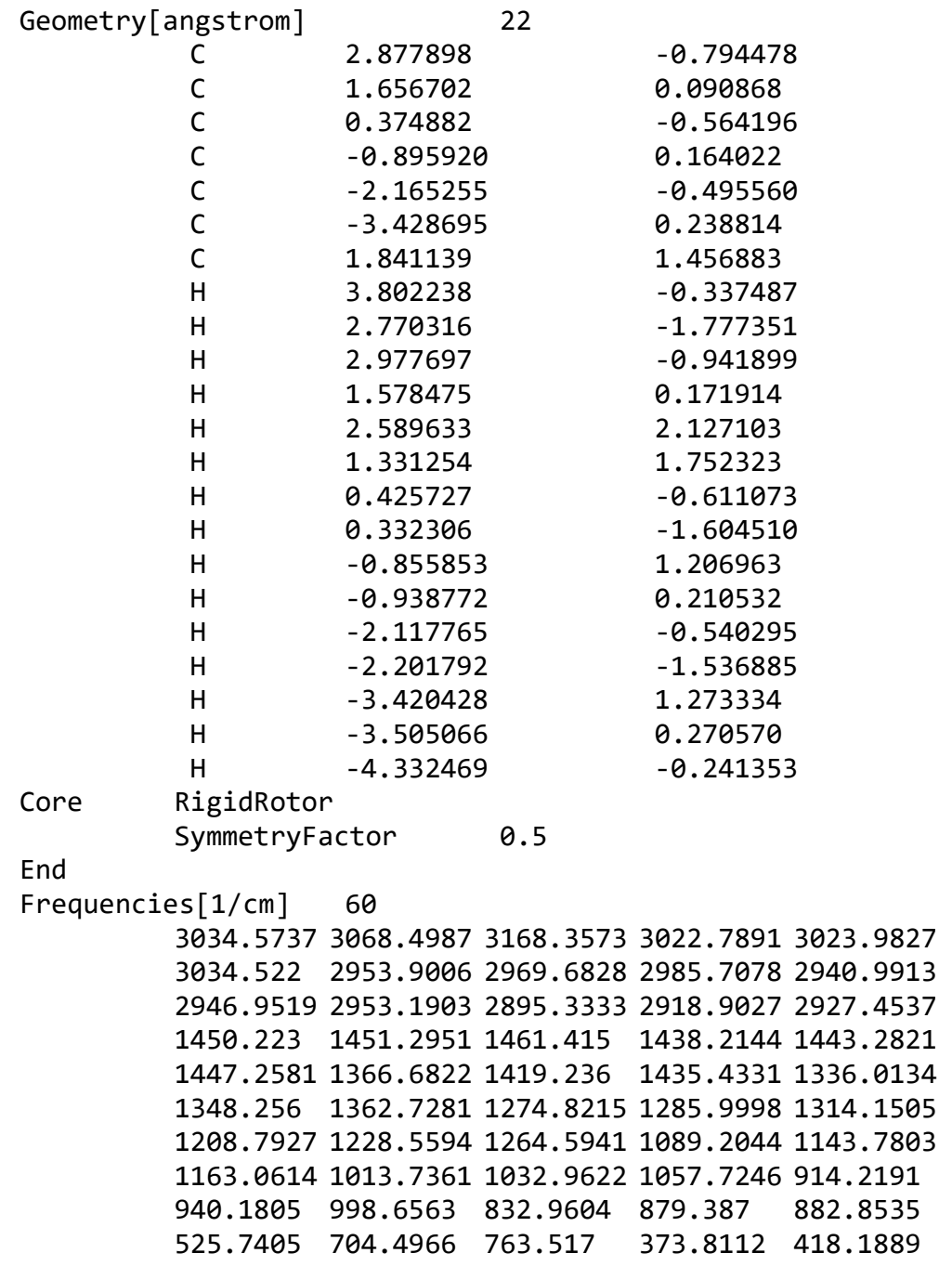

0.027662

0.333186

$-0.186344$

0.235953

$-0.292788$

0.141314

$-0.232058$

0.391760

0.498471

$-1.052897$

1.429911

0.172026

$-1.142041$

$-1.284847$

0.163433

$-0.107692$

1.333670

$-1.388538$

0.052687

$-0.217358$

1. 233171

$-0.243309$ 
End

$\begin{array}{lllll}450.8962 & 228.1196 & 282.6025 & 285.4435 & 120.0641\end{array}$

$\begin{array}{lllll}154.2477 & 223.7787 & 55.6091 & 75.2174 & 92.5654\end{array}$

ZeroEnergy[kcal/mol] $\quad-19.92656417248466$

End

Well 2

Species

RRHO

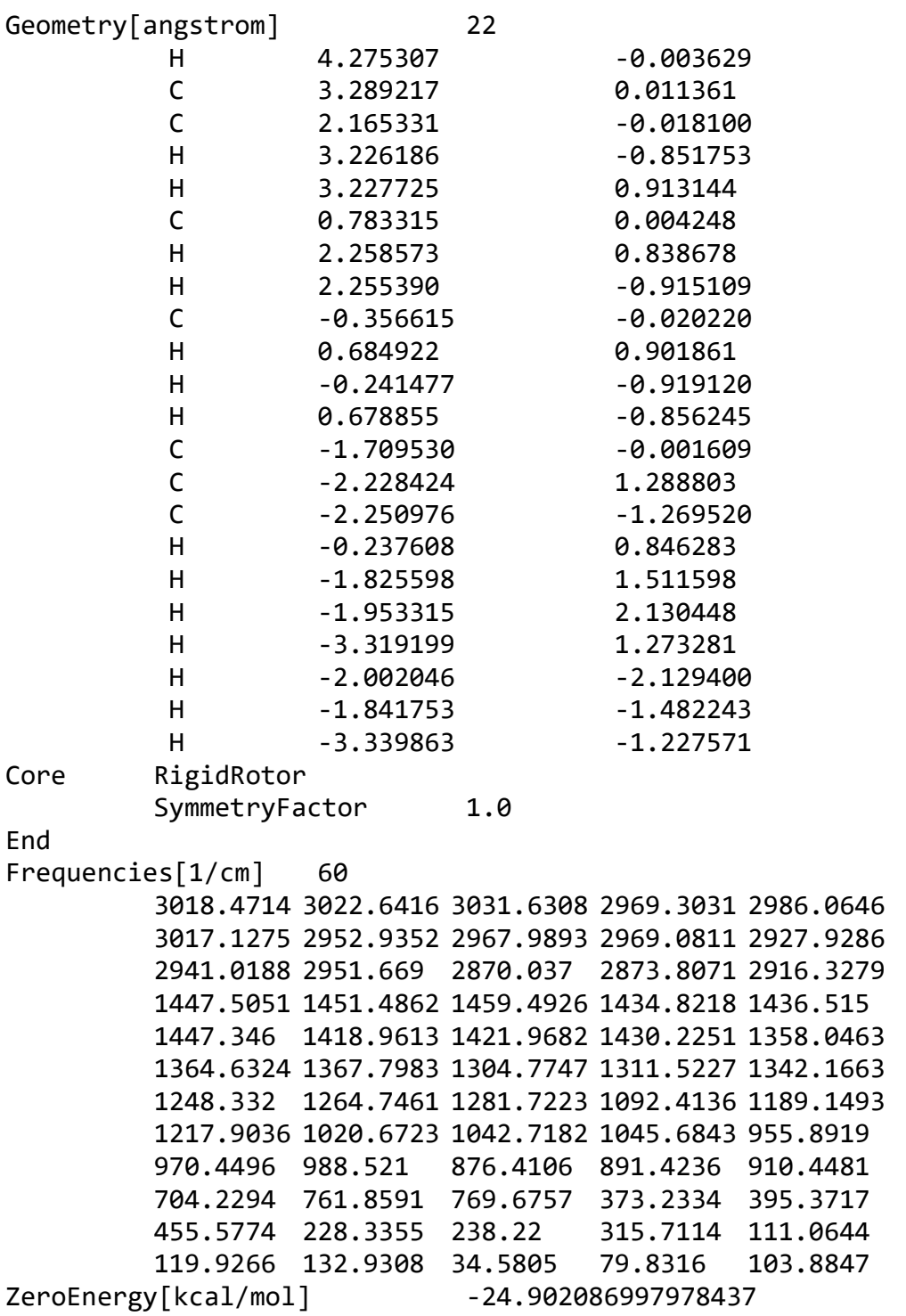

End

End .902086997978437

Well 6

Species RRHO

$\begin{array}{cl}\text { Geometry[angstrom] } & \\ \text { C } & -1.570543 \\ \text { C } & -0.263497 \\ \text { C } & 0.987746 \\ \text { C } & -2.746698 \\ \text { H } & -0.163361 \\ \text { H } & -0.332205 \\ \text { C } & 2.274107 \\ \text { H } & 0.970629 \\ \text { H } & 1.000006 \\ \text { C } & 3.502665 \\ \text { H } & 2.223621\end{array}$

22

$\begin{array}{ll}0.024556 & -0.357283 \\ -0.583827 & 0.156491 \\ 0.214357 & -0.192748 \\ -0.884701 & -0.009564 \\ -1.601148 & -0.247578 \\ -0.696195 & 1.249624 \\ -0.515603 & 0.191244 \\ 1.187031 & 0.315006 \\ 0.428821 & -1.270347 \\ 0.268361 & -0.090837 \\ -0.772729 & 1.264770\end{array}$

$-0.077987$

$-0.550075$

0.479556

$-1.220911$

$-1.168118$

$-0.163744$

1.159347

1.105759

0.870971

$-0.789977$

1.492884

$-0.839185$

0.241551

$-0.291512$

$-0.322016$

1.536912

$-1.295319$

0.353123

$-0.393225$

0.309023

$-1.325478$

$-0.435225$ 
End

$$
\begin{array}{ll}
\text { Core } & \text { RigidRotor } \\
& \text { SymmetryFactor } \quad 0.5
\end{array}
$$

End

3.454655

4.477912

$-1.499244$

$-2.852012$

$-2.604465$

$-3.687801$

$-1.801366$

$-1.031507$

$-1.796253$

$-2.770981$

2.316520

Frequencies $[1 / \mathrm{cm}] \quad 60$

$3033.8353 \quad 3080.47663179 .26013013 .919 \quad 3017.2396$

$3027.75662944 .50732956 .54 \quad 2985.4146 \quad 2932.2536$ 2935.97552941 .76462867 .54662912 .66482924 .1764

1451.13731454 .26321462 .59741428 .40851439 .9325

1444.25161372 .12071420 .47111427 .64871336 .5134

1356.36671360 .85191280 .68281292 .84411324 .5352

$\begin{array}{llllll}1184.5 & 1227.5069 & 1249.5646 & 1084.4487 & 1138.9687\end{array}$

1163.23231042 .10791044 .08911072 .6507930 .2633

$\begin{array}{lllll}942.3638 & 963.0472 & 817.0784 & 877.011 & 895.0894\end{array}$

$\begin{array}{lllll}475.77 & 705.6782 & 781.2208 & 391.5645 & 422.6816\end{array}$

$\begin{array}{llllll}452.5247 & 243.9423 & 289.8849 & 301.3371 & 125.5234\end{array}$

$\begin{array}{llllll}145.8828 & 217.5321 & 64.1131 & 82.1357 & 97.2383\end{array}$

ZeroEnergy [kcal/mol]
End

Well 3
Species

RRHO
$-0.189921$

$-0.096544$

$-1.452608$

1.078948

$-0.409022$

$-0.405359$

0.205433

$-0.116847$

1.302432

$-0.113154$

$-0.325817$
Geometry[angstrom]

C $\quad-1.939963$

1.939963

C $\quad-2.775137$

C $\quad-0.327278$

$\mathrm{H} \quad-2.036337$

C $\quad 0.930873$

C $\quad 2.193016$

C $\quad 3.466245$

$\mathrm{H} \quad-2.891224$

$\mathrm{H} \quad-2.581065$

$\mathrm{H} \quad-2.878226$

$\mathrm{H} \quad-1.560619$

$\mathrm{H}-0.302680$

H $\quad-1.144876$

H $\quad-3.724834$

$\mathrm{H} \quad 0.993294$

H $\quad 0.916960$

H 2.160871

$\mathrm{H} \quad 2.189784$

$\mathrm{H} \quad 3.524124$

$\mathrm{H} \quad 3.492468$

$\mathrm{H} \quad 4.363417$

Core

RigidRotor

End SymmetryFactor

0.5
22

1.407494
-0.005027
-0.960690
-0.505983
1.392272
0.257678
-0.591000
0.225468
-1.036870
-1.964744
1.783511
0.072904
-1.407539
2.112740
-0.609878
1.103504
0.729585
-1.410779
-1.060837
1.031477
0.687123
-0.390100

0.174400

$-0.350876$

$-0.010242$

0.164326

1.265854

$-0.042394$

0.106091

$-0.079091$

1.076987

$-0.399109$

$-0.245965$

$-1.450436$

0.769983

$-0.085166$

$-0.424824$

0.667160

$-1.038829$

$-0.621410$

1.097729

0.659917

$-1.071728$

0.026549

Frequencies [1/cm ] 59

3032.78973034 .07993085 .43823021 .24223022 .7403 3031.59322951 .59842983 .13593020 .30392944 .7315 2945.51352947 .95742857 .23492867 .62152905 .9383 1451.3851458 .75861460 .94261439 .45091444 .5562 $1451.119 \quad 1390.9198 \quad 1421.9106 \quad 1438.718 \quad 1350.0274$ 1365.79371367 .93941277 .60331287 .05221335 .2252 
End

ZeroEnergy $[\mathrm{kcal} / \mathrm{mol}] \quad-22.89656663597678$

1208.98711224 .58241274 .42871103 .25121146 .7449

1169.53471037 .35221066 .21611071 .2926931 .4085

$\begin{array}{llllll}946.5071 & 1024.1179 & 834.4672 & 879.8951 & 890.9865\end{array}$

$\begin{array}{llllll}480.0535 & 707.5549 & 813.4887 & 289.8076 & 390.2026\end{array}$

$\begin{array}{llllll}456.9936 & 226.9927 & 269.9628 & 278.5317 & 124.5008\end{array}$

$\begin{array}{llll}213.3108 & 221.7157 & 49.1715 & 94.0764\end{array}$

End

$\begin{array}{cc}\text { Bimolecular } & 13 \\ \text { Fragment } & 13 \text { m }\end{array}$

RRHO

Geometry[angstrom]

$\begin{array}{ll}\mathrm{C} & 1.227078 \\ \mathrm{H} & 1.800272\end{array}$

9

$\mathrm{H} \quad 1.800272$

1.801086

1.162752

$-0.135226$

$-0.173468$

$-1.271722$

$$
-2.235213
$$

H $\quad-1.276207$

$-22.89656663597678$

(1)

8




Geometry [an

ngstrom]

$-2.182401^{22}$

Core RigidRotor

End SymmetryFactor

-2.182401
-0.879012
0.352555
-3.411603
-2.199288
-0.873233
-0.835269
1.666968
0.280888
0.345091
2.838481
2.188973
2.693037
3.837709
-2.207397
-3.414266
-3.425536
-4.340161
1.933340
2.040210
1.131471
2.867809

$-0.879012$

C $\quad 0.352555$

$\mathrm{H} \quad-2.199288$

$\mathrm{H} \quad-0.873233$

$\mathrm{H} \quad-0.835269$

C $\quad 1.666968$

$\mathrm{H} \quad 0.280888$

$\mathrm{H} \quad 0.345091$

C $\quad 2.838481$

$\mathrm{H} \quad 2.188973$

$\mathrm{H} \quad 2.693037$

H $\quad 3.837709$

$\mathrm{H} \quad-2.207397$

$\mathrm{H} \quad-3.414266$

$\mathrm{H} \quad-3.425536$

$\mathrm{H} \quad-4.340161$

C $\quad 1.933340$

$\mathrm{H} \quad 2.040210$

$\mathrm{H} \quad 1.131471$

2.867809
C $\quad-3.411603$

Frequencies [1/cm] 59

3033.95063079 .18363194 .12192992 .25013023 .7048 3033.69492952 .86222965 .05052986 .12632929 .1924 2940.95692945 .89872151 .62952860 .29662893 .5537 1448.02921450 .19141460 .94391437 .15571441 .721 1444.10481370 .82051383 .85331422 .62841333 .6101 1363.13291364 .64561269 .35611283 .99251299 .5276 1198.98821220 .17581262 .49861089 .53951101 .7629 $1197.6702992 .0318 \quad 1023.19111055 .2902892 .2507$ $\begin{array}{llllll}915.584 & 977.1306 & 760.2537 & 795.1049 & 880.684\end{array}$ $\begin{array}{lllll}463.2815 & 706.9839 & 709.4876 & 345.7669 & 393.9225\end{array}$ $\begin{array}{llllll}417.401 & 231.4904 & 283.7068 & 300.5471 & 114.3882\end{array}$ $\begin{array}{llll}139.2784 & 201.9967 & 58.9766 & 79.2607\end{array}$

End ZeroEnergy [ $\mathrm{kcal} / \mathrm{mol}]$ 15.766176187501712
Barrier
TS_1
6

Geometry [angstrom]

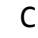

C

c

C

c

c

C

$\mathrm{H}$

$\mathrm{H}$

$\mathrm{H}$

$\mathrm{H}$

$\mathrm{H}$

$\mathrm{H}$

$\mathrm{H}$

H

$\mathrm{H}$

H

$\mathrm{H}$

H

$$
\begin{aligned}
& -0.674363 \\
& -1.156416 \\
& -2.658870 \\
& -0.390699 \\
& 1.059819 \\
& 2.051877 \\
& 1.889059 \\
& 0.632288 \\
& -0.985739 \\
& -3.227315 \\
& -3.035192 \\
& -2.860048 \\
& -0.942604 \\
& -0.417295 \\
& 1.401375 \\
& 1.087725 \\
& 1.929647 \\
& 3.074637 \\
& 2.496346 \\
& 1.915737 \\
& -0.685421 \\
& -1.106585
\end{aligned}
$$

22
0.203196

$-0.127588$

0.282638

$-0.184751$

$-0.311002$

0.374869

$-1.205663$

$-0.115861$

$-0.137126$

1.380314

$-0.166916$

$-1.207607$

$-0.017884$

$-0.114680$

1.277315

$-1.258621$

0.341583

0.053916

0.138242

1.219861

$-0.233705$

$-0.335330$ 


\begin{tabular}{|c|c|c|c|c|c|c|c|c|}
\hline & & Core & $\begin{array}{l}\text { RigidRoto } \\
\text { SymmetryF }\end{array}$ & actor & 0.5 & & & \\
\hline & & End & & & & & & \\
\hline & & Frequ & $\mathrm{s}[1 / \mathrm{cm}]$ & 59 & & & & \\
\hline & & & 3028.2736 & 3068.8049 & 3079.2459 & 2984.2075 & 2996.5115 & \\
\hline & & & 3015.8065 & 2958.3278 & 2969.1721 & 2983.2641 & 2921.8007 & \\
\hline & & & 2928.6793 & 2942.5355 & 1449.5313 & 2908.5797 & 2919.5489 & \\
\hline & & & 1436.905 & 1441.6063 & 1445.2784 & 1405.987 & 1406.2295 & \\
\hline & & & 1430.97 & 1356.4104 & 1361.4466 & 1390.9468 & 1328.8205 & \\
\hline & & & 1341.4985 & 1343.9814 & 1254.536 & 1282.2723 & 1326.2596 & \\
\hline & & & 1158.8229 & 1205.5973 & 1226.4985 & 1080.5829 & 1129.0238 & \\
\hline & & & 1140.0406 & 982.6994 & 1010.7092 & 1056.7928 & 919.2656 & \\
\hline & & & 935.053 & 973.2528 & 823.936 & 874.0447 & 906.5198 & \\
\hline & & & 591.8952 & 750.9402 & 791.1583 & 434.264 & 499.4998 & \\
\hline & & & 560.6933 & 312.2887 & 352.734 & 398.0278 & 226.0988 & \\
\hline & & & 256.3682 & 287.9882 & 83.1911 & 120.7361 & & \\
\hline & & ZeroE & / $[\mathrm{kcal} / \mathrm{mol}$ & & -2.179968 & 8002989257 & & \\
\hline & End & & & & & & & \\
\hline Barrier & TS_1_13 & 1 & 13 & & & & & \\
\hline & RRHO & & & & & & & \\
\hline & & Geome & angstrom ] & & 22 & & & \\
\hline & & & $\mathrm{C}$ & 3.011392 & & -0.73624 & & -0.064122 \\
\hline & & & C & 2.022041 & & 0.295539 & & 0.412640 \\
\hline & & & C & 0.180756 & & -0.86664 & & -0.088657 \\
\hline & & & C & -0.962531 & & 0.072189 & & 0.090566 \\
\hline & & & C & -2.333752 & & -0.57914 & & -0.139832 \\
\hline & & & C & -3.473972 & & 0.416086 & & 0.047186 \\
\hline & & & C & 1.884612 & & 1.494383 & & -0.204502 \\
\hline & & & $\mathrm{H}$ & 3.983516 & & -0.58410 & & 0.417291 \\
\hline & & & $\mathrm{H}$ & 2.687369 & & -1.75586 & & 0.161088 \\
\hline & & & $\mathrm{H}$ & 3.158819 & & -0.65533 & & -1.145363 \\
\hline & & & $\mathrm{H}$ & 1.709985 & & 0.220763 & & 1.454508 \\
\hline & & & $\mathrm{H}$ & 1.299908 & & 2.295932 & & 0.233549 \\
\hline & & & $\mathrm{H}$ & 2.305842 & & 1.666416 & & -1.191327 \\
\hline & & & $\mathrm{H}$ & 0.465225 & & -1.14309 & & -1.100416 \\
\hline & & & $\mathrm{H}$ & 0.322680 & & -1.64853 & & 0.653917 \\
\hline & & & $\mathrm{H}$ & -0.849895 & & 0.920211 & & -0.598051 \\
\hline & & & $\mathrm{H}$ & -0.941431 & & 0.496126 & & 1.105369 \\
\hline & & & $\mathrm{H}$ & -2.364613 & & -1.00212 & & -1.151158 \\
\hline & & & $\mathrm{H}$ & -2.454037 & & -1.42321 & & 0.549969 \\
\hline & & & $\mathrm{H}$ & $-3.37828 e$ & & 1.253196 & & -0.652016 \\
\hline & & & $\mathrm{H}$ & -3.466517 & & 0.830990 & & 1.060484 \\
\hline & & & $\mathrm{H}$ & -4.449856 & & -0.04839 & & -0.117514 \\
\hline & & Core & RigidRoto & & & & & \\
\hline & & & SymmetryF & actor & 0.5 & & & \\
\hline & & End & & & & & & \\
\hline & & Frequ & $\mathrm{s}[1 / \mathrm{cm}]$ & 59 & & & & \\
\hline & & & 3059.3245 & 3123.2275 & 3150.0456 & 3032.9531 & 3034.1098 & \\
\hline & & & 3047.1033 & 3018.6368 & 3023.5309 & 3029.6985 & 2952.9595 & \\
\hline & & & 2962.7938 & 2988.2556 & 2917.6557 & 2947.1145 & 2952.0536 & \\
\hline & & & 1451.3436 & 1458.4464 & 1542.1316 & 1436.0915 & 1443.8032 & \\
\hline & & & 1446.3221 & 1390.8138 & 1420.9397 & 1434.4886 & 1328.9358 & \\
\hline & & & 1357.457 & 1364.2985 & 1248.1623 & 1257.6168 & 1284.7043 & \\
\hline & & & 1146.4815 & 1168.3354 & 1242.3407 & 1022.2125 & 1062.4697 & \\
\hline & & & 1085.1471 & 927.8195 & 975.293 & 1014.0825 & 868.822 & \\
\hline & & & 895.8943 & 907.8554 & 777.5329 & 781.794 & 837.3792 & \\
\hline & & & 540.8591 & 546.3868 & 707.3431 & 305.322 & 400.7278 & \\
\hline & & & 410.9918 & 216.1383 & 235.9629 & 247.9283 & 91.2369 & \\
\hline & & & 124.822 & 163.8785 & 46.7074 & 58.4824 & & \\
\hline & & ZeroE & / $[\mathrm{kcal} / \mathrm{mo}]$ & & 9.603405 & 388009186 & & \\
\hline & End & & & & & & & \\
\hline Barrier & TS_3_6 & 3 & 6 & & & & & \\
\hline & & Geome & ngstrom ] & & 22 & & & \\
\hline & & & $\mathrm{C}$ & -2.080559 & & 1.080021 & & -0.041295 \\
\hline
\end{tabular}




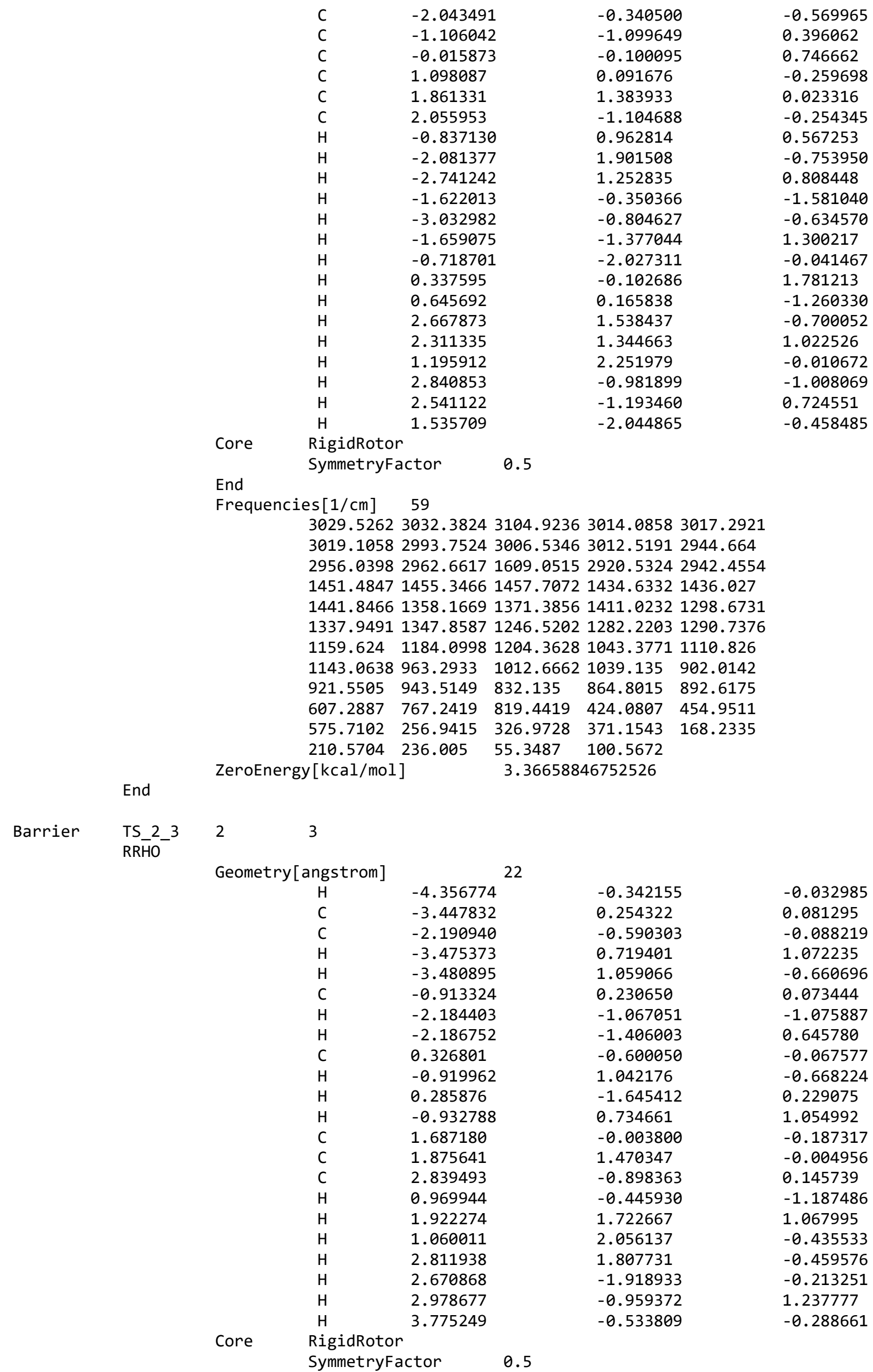


End

Frequencies [1/cm] 59

3032.93463041 .23353073 .73982993 .75123021 .3279

$3026.39352951 .54 \quad 2982.59842983 .56352893 .9579$

$\begin{array}{llllll}2933.0187 & 2944.9309 & 2137.6013 & 2878.084 & 2885.4621\end{array}$

1451.13141453 .73891460 .59841436 .39281443 .0324

1445.23041378 .01071425 .70841433 .00971363 .3234

$\begin{array}{llllll}1367.1033 & 1371.807 & 1277.7714 & 1288.339 & 1331.0479\end{array}$

$1216.814 \quad 1224.90641263 .38851084 .20251099 .8686$

$1172.49141034 .41361045 .58691063 .113 \quad 925.52$

$\begin{array}{llllll}961.6572 & 974.9463 & 786.0334 & 847.2428 & 883.9795\end{array}$

$\begin{array}{llllll}454.487 & 647.689 & 719.3427 & 291.0545 & 339.5461\end{array}$

$\begin{array}{llllll}415.0465 & 214.0379 & 225.6292 & 281.8907 & 115.2596\end{array}$

$\begin{array}{llll}147.2618 & 181.7907 & 54.2829 & 69.9263\end{array}$

End

5.557843033528325 